\title{
Vortices and Monopoles in Mass-deformed $S O$ and USp Gauge Theories
}

\author{
Minoru Eto ${ }^{1}$, Toshiaki Fujimori ${ }^{2,3}$, Sven Bjarke Gudnason ${ }^{4}$, \\ Yunguo Jiang ${ }^{5,6}$, Kenichi Konishi ${ }^{3,2}$, Muneto Nitta ${ }^{7}$, Keisuke Ohashi ${ }^{8}$ \\ ${ }^{1}$ Department of Physics, Yamagata University, Yamagata 990-8560, Japan \\ 2 INFN, Sezione di Pisa, Largo B. Pontecorvo, 3, 56127 Pisa, Italy \\ 3 Department of Physics, "E. Fermi", University of Pisa, Largo B. Pontecorvo, 3, 56127 Pisa, Italy \\ 4 Racah Institute of Physics, The Hebrew University, Jerusalem 91904, Israel \\ 5 Institute of High Energy Physics, Chinese Academy of Sciences, 100049 Beijing, China \\ 6 Theoretical Physics Center for Science Facilities, Chinese Academy of Sciences, 100049 Beijing, \\ China \\ 7 Department of Physics, and Research and Education Center for Natural Sciences, \\ Keio University, Hiyoshi 4-1-1, Yokohama, Kanagawa 223-8521, Japan \\ 8 Department of Physics, Kyoto University, Kyoto 606-8502, Japan
}

\begin{abstract}
Effects of mass deformations on 1/2 Bogomol'nyi-Prasad-Sommerfield (BPS) non-Abelian vortices are studied in $4 d \mathcal{N}=2$ supersymmetric $U(1) \times S O(2 n)$ and $U(1) \times U S p(2 n)$ gauge theories, with $N_{f}=2 n$ quark multiplets. The $2 d \mathcal{N}=(2,2)$ effective worldsheet sigma models on the Hermitian symmetric spaces $S O(2 n) / U(n)$ and $U S p(2 n) / U(n)$ found recently which describe the low-energy excitations of the orientational moduli of the vortices, are generalized to the respective massive sigma models. The continuous vortex moduli spaces are replaced by a finite number $\left(2^{n-1}\right.$ or $\left.2^{n}\right)$ of vortex solutions. The $1 / 2$ BPS kinks connecting different vortex vacua are magnetic monopoles in the $4 d$ theory, trapped inside the vortex core, with total configurations being 1/4 BPS composite states. These configurations are systematically studied within the semi-classical regime.
\end{abstract}




\section{Contents}

1 Introduction

2 The bulk theory

2.1 The Model . . . . . . . . . . . . . . . . . . . . . . .

2.2 Orientational zero modes of the non-Abelian vortices . . . . . . . . . . . 6

2.3 Mass deformation . . . . . . . . . . . . . . . . . . . . 12

3 Mass-deformed sigma model

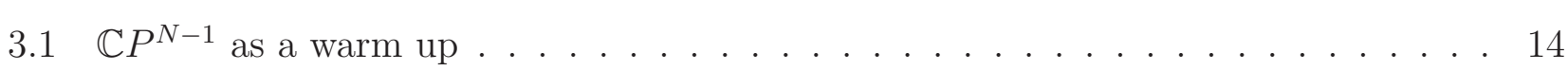

3.2 The $S O(2 n) / U(n)$ and $U S p(2 n) / U(n)$ sigma models $\ldots \ldots \ldots \ldots$

4 Monopoles as kinks on the vortex $\quad 17$

$4.1 S U(2)$ monopole . . . . . . . . . . . . . . . . . . . 17

$4.2 S O(2 n)$ and $U S p(2 n)$ monopoles $\ldots \ldots \ldots \ldots \ldots \ldots$

4.2 .1 BPS kink equations . . . . . . . . . . . . . . . . 19

4.2 .2 Vacua on the vortex worldsheet . . . . . . . . . . . . . 23

4.2 .3 Single monopole configurations . . . . . . . . . . . . . . . . . . 26

4.2.4 Multi-monopole configurations . . . . . . . . . . . . . . . 31

5 Witten's effect on the vortex worldsheet

6 Summary

\begin{tabular}{l|l|} 
A Domain wall & 44 \\
\hline
\end{tabular}

B Lie algebra

B.1 $G=S U(N) \ldots \ldots \ldots \ldots \ldots \ldots \ldots \ldots \ldots \ldots \ldots \ldots \ldots \ldots \ldots \ldots$

B.2 $G=S O(2 n) \ldots \ldots \ldots \ldots \ldots \ldots \ldots \ldots \ldots \ldots \ldots \ldots \ldots \ldots \ldots \ldots \ldots \ldots \ldots \ldots$

B.3 $G=U S p(2 n) \ldots \ldots \ldots \ldots \ldots \ldots \ldots \ldots \ldots \ldots \ldots \ldots \ldots \ldots \ldots \ldots \ldots$ 


\section{Introduction}

The discovery of non-Abelian vortex solutions [1, 2] and the subsequent development [3]-[20] have led to a substantial improvement of our understanding of non-Abelian solitons in general, appearing in spontaneously broken gauge theories. Among the techniques used to explore these objects are: the string theory construction due to Hanany-Tong [1] which in turn gave rise to the Kähler quotient construction, the powerful moduli matrix method [10]-[20] (the determination of the moduli space [10], the moduli space metric [12], low-energy dynamics [11, 13], generalization to arbitrary gauge groups [14]-[16], etc), apart from the standard field-theoretic analyses [2]-[9], have all proven invaluable.

The effective theories of the fluctuations of non-Abelian vortex orientational modes have been derived in the field-theoretic approach [2], [6]-[8] as well as from string theory [1]. In theories with gauge group $U(N)$ and $N_{f}=N$ flavors (hypermultiplets), the worldsheet effective action turns out to be a two-dimensional $\mathbb{C} P^{N-1}$ sigma model.

A particularly interesting result concerns the precise matching of the BPS spectra of the four dimensional gauge theories (having $\mathcal{N}=2$ supersymmetry) under study and the two-dimensional sigma models (having $\mathcal{N}=(2,2)$ supersymmetry) [21]. In a sense the Abelian monopoles in the Coulomb phase become trapped inside a vortex in the Higgs phase [3], [5]-[8]. The monopoles are realized as classical kinks along the vortex string when the unequal squark mass terms are introduced. This in turn induces a potential on the sigma model, which has now $N$ vacua instead of a continuous $\mathbb{C} P^{N-1}$ degeneracy.

In the theory without the bare quark masses, $N$ vacua appear as a result of quantum effects. The kinks interpolating different (vortex) vacua correspond to the light monopoles appearing as the singularities of the Seiberg-Witten curves. More precisely, these $N$ vortex vacua and the light monopoles connecting them correspond to the physics of the quantum $r=0$ vacua, arising from the classical $r=N$ vacua of the theory with $N_{f}=N$ massless flavors [22]-[24].

In a recent development [9], the worldsheet low-energy effective actions of non-Abelian vortices in gauge theories with $U(1) \times S O(2 n)$ or $U(1) \times U S p(2 n)$ gauge symmetry and $N_{\mathrm{f}}=2 n$ flavors (hypermultiplets) [14]-[16] have been found explicitly. The effective theories found are non-linear 
sigma models on the Hermitian symmetric spaces (HSS): $S O(2 n) / U(n)$ and $U S p(2 n) / U(n)$, respectively [14]. The construction of the effective action has been extended to some higherwinding vortices in $U(1) \times S O(2 n)$ as well as in $U(N)$ theories, and in all cases the results found agreed with those obtained in a more formal approach based on symmetry and group-theoretic considerations [25]. The results found reduce to the known $\mathbb{C} P^{N-1}$ effective action [3, 5, 6, 8, in the case of the $U(N)$ theory.

In this paper we will extend these non-linear sigma models to the case of the mass-deformed theories. As in the $\mathbb{C} P^{N-1}$ models, a potential is induced on the sigma model. Such a potential can be obtained either in the bulk theory, or alternatively, in the worldsheet effective theory by using the Scherk-Schwarz (SS) dimensional reduction 1 We then construct 1/4 BPS configuration of magnetic monopoles confined in a vortex-string as kinks in the vortex world-sheet effective theory with mass deformation. By generalizing the operator method to create domain walls in the case of $S U(N)$ gauge theory [18] (in which the operators are identified with the root vectors [28]), we find the rule of ordering of kinks (monopoles on the vortex) in SO and USp gauge theories.

The paper is organized as follows. In Section 2 we review the non-Abelian vortex solutions and their orientational zero modes in the bulk gauge theory and derive the vortex worldsheet effective action. These serve as the starting point for the discussion of the mass-deformed systems and the derivation of the massive sigma models in Section 3 , Section 4 is dedicated to the analysis of the Abelian monopoles which appear along the vortex string as kinks.2 A comment on the solutions involving domain walls in the bulk is given in Appendix A. The convention used for the Lie algebra is reviewed in Appendix B, and some useful results are collected in Appendix C. The case of the effective worldsheet action for certain doubly wound vortices in $S O(2 n)$ theory, which is given by a quadric surface $Q^{2 n-2}$ sigma model, is discussed briefly in Appendix D.

\footnotetext{
${ }^{1}$ Our analysis follows the method [7] developed to obtain the mass-deformed theory in four space-time dimensions from a five space-time dimensional massless theory by the Scherk-Schwarz dimensional reduction [26, 27.

2 When this paper was being written, a paper by Arai and Shin [29] appeared which discusses the massdeformed sigma models in four (or three) dimensions, without any reference to the non-Abelian vortices, but based on a similar model with $S O(2 n)$ and $U S p(2 n)$ global symmetries. Their analysis of the domain walls naturally have some overlap with our analysis of the kink monopoles and, where the comparison is possible, our results seem to agree.
} 


\section{The bulk theory}

\subsection{The Model}

The theory we are considering is the standard $\mathcal{N}=2$ supersymmetric QCD with gauge group $U(1) \times G$ with $G=S U(N), S O(2 n)$ and $U S p(2 n)$ (while $N$ denotes in all cases the dimension of the fundamental representation, i.e. $N=2 n$ for $S O, U S p)$. The F-terms are given by variations of the following superpotential

$$
\mathcal{W}=\operatorname{Tr}[\tilde{Q} \Phi Q-\tilde{Q} Q M]
$$

where $Q, \tilde{Q}$ are fundamental and anti-fundamental chiral superfields comprising the $N_{\mathrm{f}}$ hypermultiplets ( $Q$ is an $N$-by- $N_{\mathrm{f}}$ matrix, and $\tilde{Q}$ is an $N_{\mathrm{f}}$-by- $N$ matrix), while $\Phi$ is a chiral superfield belonging to a vector multiplet, in the adjoint representation. In this paper we will set the $N_{\mathrm{f}}$ by- $N_{\mathrm{f}}$ mass matrix to be real: $M^{\dagger}=M$. Using a flavor rotation we can diagonalize the mass matrix as $M=\operatorname{diag}\left(m_{1}, \cdots, m_{N_{\mathrm{f}}}\right)$. Furthermore, the trace part of $M$ can be absorbed by a shift of the adjoint scalar, so that we can assume that $\operatorname{Tr} M=0$ without loss of generality.

We shall restrict ourselves to the case of $N_{\mathrm{f}}=N$ flavors, for definiteness. This is the minimum number of flavors which allows for a color-flavor locked vacuum. In the massless case, then, the theory has an $S U(N)$ flavor symmetry, in all cases, including $U(1) \times S O(2 n)$ or $U(1) \times U S p(2 n)$, with $N_{f}=N=2 n$ flavors.

Finally, we can set $\tilde{Q}=0$ and $\Phi^{\dagger}=\Phi$ for the consideration of the non-Abelian vortex solutions and small excitations around them.

Due to renormalization group (RG) flow, the gauge coupling constants for $U(1)$ and for $G$ would become distinct at a lower energy scale even if we start with an equal, common coupling constant. However, as our results here do not substantially depend on the $U(1)$ gauge coupling and also because our analysis remains semi-classical throughout, we shall set $e=g$ for simplicity.

The bosonic part of the Lagrangian density is then

$$
\mathcal{L}=\operatorname{Tr}\left[-\frac{1}{2 g^{2}} F_{\mu \nu} F^{\mu \nu}-\frac{\theta}{16 \pi^{2}} F_{\mu \nu} \tilde{F}^{\mu \nu}+\frac{1}{g^{2}}\left(\mathcal{D}_{\mu} \Phi\right)^{2}+\left|\mathcal{D}_{\mu} Q\right|^{2}\right]-V_{D}-V_{F},
$$

where we denote the scalar components of the superfields with the same symbols as the superfields themselves. The scalar potentials read

$$
\begin{aligned}
& V_{D}=\frac{g^{2}}{2}\left|\operatorname{Tr}\left(Q Q^{\dagger} t^{\alpha}\right)-\xi^{\alpha}\right|^{2}, \\
& V_{F}=\operatorname{Tr}|\Phi Q-Q M|^{2},
\end{aligned}
$$


where $\alpha=0,1, \ldots, \operatorname{dim}(G)$ and 0 stands for the $U(1)$ part of the gauge group. $\xi^{0}=\xi$ is the only non-zero Fayet-Iliopoulos (FI) parameter as $G$ is a simple group. Finally all generators are normalized as $\operatorname{Tr}\left(t^{\alpha} t^{\beta}\right)=\delta^{\alpha \beta} / 2$.

Since the mass matrix $M$ is diagonal and traceless, it belongs to an element of the Cartan subalgebra of $G$. There exists a Higgs vacuum in which the vacuum expectation values (VEVs) of the scalar fields take the form

$$
\langle\Phi\rangle=M, \quad\langle Q\rangle=v \mathbf{1}_{N}, \quad\left(v \equiv \sqrt[4]{\frac{2}{N}} \sqrt{\xi}\right) .
$$

In this paper we focus our attention on this system.

For the massless case $M=0$, the VEV of the adjoint scalar $\Phi$ does not break the $U(1) \times G$ gauge symmetry, while the squark VEV breaks it completely, bringing the system in the Higgs phase. However, the global color-flavor diagonal $G_{\mathrm{C}+\mathrm{F}}$ symmetry remains intact.

In the theory with a generic bare mass-matrix $M$, the gauge group is broken to $U(1)^{r}$ where $r=\operatorname{rank} G$. As already mentioned, $M$ can be rotated into a diagonal form without loss of generality. For the $G=S U(N)$ theory, the generic mass matrix $M$ takes the form $M=\operatorname{diag}\left(m_{1}, \cdots, m_{N}\right)$. While in the cases of $G=S O(2 n)$ and $G=U S p(2 n)$, we will be working in the usual basis $U^{\mathrm{T}} J U=J$, with $J=\sigma^{1} \otimes \mathbf{1}_{n}$ and $J=i \sigma^{2} \otimes \mathbf{1}_{n}$, respectively. In this basis the mass matrix reads $M=\operatorname{diag}\left(m_{1}, \cdots, m_{n},-m_{1}, \cdots,-m_{n}\right)$. The properties of the solitons will be seen to depend crucially on how the components of the mass matrix $M$ is chosen.

There are several kinds of topological solitons in our models. Among them we are interested in vortices and monopoles. The $U(1)$ symmetry breaking at the energy $\langle Q\rangle \sim v$ gives rises to vortices, while the breaking of $G \rightarrow U(1)^{r}$ at the energy $\langle\Phi\rangle \sim m$ gives rises to monopoles. The static 1/4 BPS equations for composite states of the vortex and monopole [19] can easily be found to be

$$
\begin{aligned}
0 & =\mathcal{D}_{\bar{z}} Q \\
0 & =\mathcal{D}_{3} Q+\Phi Q-Q M, \\
0 & =\mathcal{D}_{\bar{z}} \Phi+i F_{\bar{z} 3}=\left[\mathcal{D}_{\bar{z}}, \Phi+\mathcal{D}_{3}\right], \\
0 & =\mathcal{D}_{3} \Phi-F_{12}+g^{2}\left[\operatorname{Tr}\left(Q Q^{\dagger} t^{\alpha}\right) t^{\alpha}-\xi t^{0}\right],
\end{aligned}
$$

where $\mathcal{D}_{\bar{z}}=\frac{1}{2}\left(\mathcal{D}_{1}+i \mathcal{D}_{2}\right)$ and we have chosen the vortex string to lie on the $x_{3}$-axis. It is well known that the last two equations describe t'Hooft-Polyakov monopoles in the Coulomb phase, $Q=\xi=0$ while the first and the third equations describe vortex solutions if $A_{3}=\Phi=M=0$. The second equation, therefore, describes the kinks regarded as monopoles along the vortex 
string which appear in the Higgs phase $\xi \neq 0$ of the mass-deformed theory. When these first order differential equations are obeyed, the Bogomol'nyi bound will be saturated

$$
\mathcal{E}=\operatorname{Tr}\left[\frac{1}{g^{2}} \epsilon_{i j k} \partial_{i}\left(\Phi F_{j k}\right)-2 \xi F_{12} t^{0}+2 \xi \partial_{3} \Phi t^{0}-i \epsilon_{n m} \partial_{n}\left[\left(\mathcal{D}_{m} Q\right) Q^{\dagger}\right]-\partial_{3}\left[(\Phi Q-Q M) Q^{\dagger}\right]\right]
$$

where $i, j, k=1,2,3$; while $n, m=1,2$ denote the directions in the transverse plane. The first two terms in the energy density describe the monopole and vortex, respectively; the others are boundary terms.

The 1/4 BPS equations (2.6)-(2.9) can be formally solved in terms of the moduli matrix [10, 19, 20]. The first equation describes distributions of $Q$ in the $x_{1}-x_{2}$ plane and the second one determines the $x_{3}$-dependence of $Q$ for a given set of $A_{i}$ and $\Phi$. Note that the third equation is just an integrability condition which tells us only that the first two equations can be solved simultaneously. The integration constants for $Q$ can be summarized in an $N$-by- $N_{f}$ holomorphic matrix in the complex coordinate $z=x_{1}+i x_{2}$, which is called the moduli matrix. With this moduli matrix, the last equation can only be solved numerically and tells us about the magnetic flux distribution in the plane transverse to the vortex string. In this paper, however, we do not treat the $1 / 4$ BPS equations and their solutions directly. Note that the integrability condition (2.8) allows us to regard the second equation as a description of the $x_{3}$-dependence for the moduli of the vortices. We will consider $1 / 2$ BPS kink solutions in an effective theory on $1 / 2$ BPS vortices corresponding to the $1 / 4$ BPS composite states. We should remark that the $1 / 4$ BPS equations (2.6)-(2.9) admit, in general, domain walls and 1/4 BPS composite states of vortices stretched between domain walls [19, 20, 30], when the VEVs of the scalar fields are different at $x_{3} \rightarrow-\infty$ and $x_{3} \rightarrow \infty$. Furthermore, the BPS equations admit $1 / 2$ semi-local [15] and fractional vortices [31]. In the rest of this paper, we do not discuss such configurations and consider only configurations of local vortices and monopoles which are invariant under rotations in the $x_{1}-x_{2}$ plane. The precise condition for the absence of domain walls, semi-local and fractional vortices can be found in Appendix A,

\subsection{Orientational zero modes of the non-Abelian vortices}

Let us first consider the $1 / 2$ BPS solutions of local-vortex strings without monopoles in the massless case, $M=0$, for which $\Phi=0, A_{3}=0$ and $\partial_{3}=0$. Here only the first and the last equations in the $1 / 4$ BPS equations remain non-trivial. We impose the following boundary condition for the scalar field $Q$ and the gauge field $A_{\theta}$ such that it has non-trivial winding in the 
gauge orbit of the scalar VEV

$$
Q \rightarrow v \exp (i \theta \lambda), \quad A_{\theta} \rightarrow-\frac{1}{r} \lambda \quad(r \rightarrow \infty)
$$

where $r e^{i \theta}=x_{1}+i x_{2} . \lambda$ is an $N$-by- $N$ constant matrix, which can be diagonalized as

$$
\lambda \rightarrow U \lambda U^{\dagger}=\nu_{0} \mathbf{1}_{N}+\tilde{\boldsymbol{\nu}} \cdot \mathbf{H}, \quad U \in G
$$

where $\mathbf{H}=\left(H_{1}, H_{2}, \cdots, H_{r}\right)$ is a basis of the Cartan subalgebra of $G$ (see Appendix $\mathrm{B}$ for the conventions for the Lie algebra). Since the scalar field $Q$ is a single-valued function, all the eigenvalues of $\lambda$ should be integers. This condition is satisfied only if $\nu_{0}$ is quantized in the following way [14]

$$
\nu_{0}=k \times\left\{\begin{array}{cl}
\frac{1}{N} & \text { for } G=S U(N) \\
\frac{1}{2} & \text { for } G=S O(2 n) \quad, \quad k \in \mathbb{Z} . \\
\frac{1}{2} & \text { for } G=U S p(2 n)
\end{array}\right.
$$

The integer $k$ corresponds to the vortex winding number classifying the topological sectors of the vortex configurations. In addition to this quantization condition for $\nu_{0}$, the single-valuedness condition is satisfied only when the coefficient vector $\tilde{\boldsymbol{\nu}}$ is a coweight, namely $\tilde{\boldsymbol{\nu}}$ should be one of the weight vectors of the dual group $\widetilde{G}$. Here $\widetilde{G}$ is the dual group whose root vectors $\tilde{\boldsymbol{\alpha}}_{i}$ are related to those of $G$ as

$$
\tilde{\boldsymbol{\alpha}}_{i}=2 \frac{\boldsymbol{\alpha}_{i}}{\boldsymbol{\alpha}_{i} \cdot \boldsymbol{\alpha}_{i}} .
$$

For the single winding configurations $(k=1)$, the vector $\tilde{\boldsymbol{\nu}}$ is given by 3

$$
\tilde{\boldsymbol{\nu}}=\text { weight vector of }\left\{\begin{array}{lll}
\mathbf{N} & \text { (fundamental rep. of } S U(N)) & \text { for } G=S U(N) \\
\mathbf{2}^{\mathbf{n}-\mathbf{1}} & \text { (Weyl spinor rep. of } S O(2 n)) & \text { for } G=S O(2 n) \\
\mathbf{2}^{\mathbf{n}} & \text { (spinor rep. of } S O(2 n+1)) & \text { for } G=U S p(2 n)
\end{array} .\right.
$$

If we choose the highest weight vector $\tilde{\boldsymbol{\nu}}_{h}$ of each representation, the diagonal matrix $\lambda_{h}=$ $\nu_{0} \mathbf{1}_{N}+\tilde{\boldsymbol{\nu}}_{h} \cdot \mathbf{H}$ takes the form

$$
\lambda_{h}=\left(\begin{array}{l|l}
\mathbf{1}_{p} & \\
\hline & \mathbf{0}_{q}
\end{array}\right), \quad(p, q)=\left\{\begin{array}{cl}
(1, N-1) & \text { for } S U(N) \\
(n, n) & \text { for } S O(2 n) \\
(n, n) & \text { for } U S p(2 n)
\end{array}\right.
$$

\footnotetext{
${ }^{3}$ In the case of $G=S O(2 n)$, there are two disjoint topological sectors classified by $\mathbb{Z}_{2}$ topological charge [16]. For $k=1$, the vortices with different $\mathbb{Z}_{2}$ charges are characterized by the weight vectors of the Weyl spinors with opposite chirality.
} 
where the subscript of $\lambda_{h}$ stands for the highest weight vector, which we denote by $\tilde{\boldsymbol{\nu}}_{h}$. For the other weight vectors, the matrix $\lambda_{h}$ can be obtained by using the transformations which exchange the eigenvalues of $\lambda$, i.e. the Weyl group elements of $G$. For the matrices $\lambda_{h}$ given above, the basic single winding vortex solution takes the following form

$$
\begin{aligned}
Q & =v\left(\begin{array}{l|l}
z e^{-\frac{1}{2} \psi} \mathbf{1}_{p} & \\
\hline & \mathbf{1}_{q}
\end{array}\right)=v \exp \left[\left(\log z-\frac{1}{2} \psi\right) \lambda_{h}\right], \\
A_{\bar{z}} & =-\frac{i}{2}\left(\begin{array}{l|l}
\partial_{\bar{z}} \psi \mathbf{1}_{p} & \\
\hline & \mathbf{0}_{q}
\end{array}\right)=-\frac{i}{2} \partial_{\bar{z}} \psi \lambda_{h},
\end{aligned}
$$

where $z=x_{1}+i x_{2}$ and $\psi$ is a smooth real function satisfying

$$
4 \partial_{z} \partial_{\bar{z}} \psi=g^{2} v^{2}\left(1-|z|^{2} e^{-\psi}\right)
$$

with $\psi \rightarrow \log |z|^{2}$ at spatial infinity. From the solution (2.17)-(2.18) and the asymptotic behavior of the function $\psi$, we can see that the matrix $\lambda_{h}$ is nothing but the magnetic flux of the vortex

$$
\int d x_{1} d x_{2} F_{12}=-2 \pi \lambda_{h}, \quad\left(F_{12}=-2 \partial_{z} \partial_{\bar{z}} \psi \lambda_{h}\right)
$$

In the massless theory $(M=0)$, there exists an unbroken color-flavor symmetry $G_{C+F}$ in the vacuum. Therefore we can obtain a set of vortex solutions by rotating the configuration (2.17)(2.18) as

$$
Q \rightarrow U^{\dagger} Q U, \quad A_{\bar{z}} \rightarrow U^{\dagger} A_{\bar{z}} U, \quad U \in G_{C+F}
$$

This set of solutions is parametrized by the orientational moduli, which are the coordinates of a coset space of the form

$$
\mathcal{M}_{\text {orientation }} \cong G / H
$$

where $H$ is a subgroup of $G$ which does not change the solution (2.17)-(2.18), that is

$$
h \in H \Longleftrightarrow h^{\dagger} \lambda_{h} h=\lambda_{h} .
$$

The orientational moduli of the vortices can be interpreted as the Nambu-Goldstone zero modes arising from the breaking of the color-flavor symmetry $G_{C+F}$ due to the vortex configuration. For the single winding vortex in the $G=S U(N), S O(2 n)$ and $U S p(2 n)$ theories, the orientational moduli spaces are given by [14]

$$
\mathcal{M}_{\text {orientation }} \cong \frac{S U(N)}{S U(N-1) \times U(1)}, \quad \frac{S O(2 n)}{S U(n) \times U(1)}, \quad \frac{U S p(2 n)}{S U(n) \times U(1)} .
$$


The physical meaning of these orientational moduli can be seen from the magnetic flux (2.20). Under the color-flavor global symmetry $G_{C+F}$, the magnetic flux transforms as

$$
\lambda_{h} \rightarrow \lambda=U^{\dagger} \lambda_{h} U, \quad U \in G
$$

This implies that the orientational moduli parametrize the internal direction of the vortex magnetic flux in the Lie algebra of the gauge group $G$.

Since the subgroup $H$ is unbroken, not all parameters in the matrix $U$ correspond to physical zero modes of the vortex. We can parametrize the coset space as follows. Let $E_{-\alpha}$ be the lowering operators in the Lie algebra of $G$ for which the generator $\lambda_{h}$ has negative eigenvalues, namely

$$
\left[\lambda_{h}, E_{-\boldsymbol{\alpha}}\right]=-\left(\tilde{\boldsymbol{\nu}}_{h} \cdot \boldsymbol{\alpha}\right) E_{-\boldsymbol{\alpha}}, \quad \tilde{\boldsymbol{\nu}}_{h} \cdot \boldsymbol{\alpha}>0
$$

Note that in our convention $\left[\mathbf{H}, E_{-\boldsymbol{\alpha}}\right]=-\boldsymbol{\alpha} E_{-\boldsymbol{\alpha}}$. Then the generic matrix $U \in G$ containing only physical parameters (i.e. called the reducing matrix) can be constructed as follows

$$
U=g_{u} g_{l}, \quad g_{l}=\exp \left(\sum_{\tilde{\boldsymbol{\nu}}_{h} \cdot \boldsymbol{\alpha}_{i}>0} b^{i} E_{-\boldsymbol{\alpha}_{i}}\right) .
$$

The parameters $b^{i}$, which are associated with the root vectors $\boldsymbol{\alpha}_{i}$, can be interpreted as the complex coordinates which parametrize the orientational moduli space. These parameters cover the coordinate patch containing the point on the moduli space which corresponds to the BPS configuration (2.17)-(2.18). The matrix $g_{u}$ is an element of the group $P$, (a parabolic subgroup of $G^{\mathbb{C}}$ ) generated by the Cartan generators $\mathbf{H}$, all the raising operators $E_{\boldsymbol{\alpha}}$ and the lowering operators $E_{-\boldsymbol{\alpha}}$ with $\tilde{\boldsymbol{\nu}}_{h} \cdot \boldsymbol{\alpha}=0$. Formally, the matrix $g_{u}$ can be written as

$$
g_{u}=\exp \left[\boldsymbol{\theta} \cdot \mathbf{H}+\sum_{\boldsymbol{\alpha}_{i} \in \Delta^{+}} c^{i} E_{\boldsymbol{\alpha}_{i}}+\sum_{\tilde{\boldsymbol{\nu}}_{h} \cdot \boldsymbol{\alpha}_{i}=0} d^{i} E_{-\boldsymbol{\alpha}_{i}}\right] \in P
$$

where $\Delta^{+}$denotes the set of all positive roots. For a given set of parameters $b^{i}$, the matrix $g_{u}$ can be determined up to $H$ transformations $g_{u} \rightarrow h g_{u}$ from the unitarity condition $U U^{\dagger}=\mathbf{1}_{N}$, that is

$$
g_{u}^{\dagger} g_{u}=\left(g_{l} g_{l}^{\dagger}\right)^{-1}
$$

Note that the choice of $h \in H$ is not important since it does not change the BPS vortex configuration. For the generator $\lambda_{h}$ in Eq. (2.16), the matrix $\sum_{\tilde{\boldsymbol{\nu}} \cdot \boldsymbol{\alpha}_{i}>0} b^{i} E_{-\boldsymbol{\alpha}_{i}}$ takes the lower triangular matrix form

$$
\sum_{\tilde{\boldsymbol{\nu}} \cdot \boldsymbol{\alpha}_{i}>0} b^{i} E_{-\boldsymbol{\alpha}_{i}}=\left(\begin{array}{c|c}
\mathbf{0}_{p} & \\
\hline B & \mathbf{0}_{q}
\end{array}\right) .
$$


The $q$-by- $p$ matrix $B$ is an $(N-1)$-vector for $G=S U(N)$ and an anti-symmetric and symmetric $n$-by- $n$ matrix for $G=S O(2 n)$ and $U S p(2 n)$, respectively

$$
B=\left(\begin{array}{c}
b_{1} \\
b_{2} \\
\vdots \\
b_{N-1}
\end{array}\right), \quad\left(\begin{array}{cccc}
0 & b_{12} & \cdots & b_{1 n} \\
-b_{12} & 0 & \ddots & \vdots \\
\vdots & \ddots & \ddots & b_{n-1, n} \\
-b_{1 n} & \cdots & -b_{n-1, n} & 0
\end{array}\right), \quad\left(\begin{array}{cccc}
b_{11} & b_{12} & \cdots & b_{1 n} \\
b_{12} & b_{22} & \ddots & \vdots \\
\vdots & \ddots & \ddots & b_{n-1, n} \\
b_{1 n} & \cdots & b_{n-1, n} & b_{n n}
\end{array}\right) .
$$

Then, the matrix $g_{l}$ is given by

$$
g_{l}=\mathbf{1}_{N}+\sum_{\tilde{\boldsymbol{\nu}} \cdot \boldsymbol{\alpha}_{i}>0} b^{i} E_{-\boldsymbol{\alpha}_{i}}=\left(\begin{array}{c|c}
\mathbf{1}_{p} & 0 \\
\hline B & \mathbf{1}_{q}
\end{array}\right),
$$

where we have used that $\left(\sum b^{i} E_{-\boldsymbol{\alpha}_{i}}\right)^{2}=0$. The corresponding matrix $g_{u}$ takes the following form

$$
g_{u}=\left(\begin{array}{c|c}
\mathbf{1}_{p} & -B^{\dagger} \\
\hline 0 & \mathbf{1}_{q}
\end{array}\right)\left(\begin{array}{c|c}
X^{\frac{1}{2}} & \\
\hline & Y^{-\frac{1}{2}}
\end{array}\right),
$$

where $X^{\frac{1}{2}}$ and $Y^{-\frac{1}{2}}$ are invertible Hermitian matrices defined by

$$
X=\mathbf{1}_{p}+B^{\dagger} B, \quad Y=\mathbf{1}_{q}+B B^{\dagger} .
$$

Therefore the matrix $U$ containing only the physical parameters $b^{i}$ takes the form

$$
U=\left(\begin{array}{c|c}
\mathbf{1}_{p} & -B^{\dagger} \\
\hline 0 & \mathbf{1}_{q}
\end{array}\right)\left(\begin{array}{c|c}
X^{\frac{1}{2}} & \\
\hline & Y^{-\frac{1}{2}}
\end{array}\right)\left(\begin{array}{c|c}
\mathbf{1}_{p} & 0 \\
\hline B & \mathbf{1}_{q}
\end{array}\right) .
$$

Such a matrix has been introduced earlier and termed the reducing matrix [32].

Next, let us discuss the effective action for the orientational moduli by using the matrix given in Eq. (2.35). At a sufficiently low energy scale, the dynamics of the zero modes is described by a non-linear sigma model on the moduli space and its effective action can be obtained as follows. The fluctuations of the orientational zero modes along the vortex worldsheet are represented by the matrix $U\left(t, x_{3}\right) \in G$ which depends weakly on the worldsheet coordinate $t$ and $x_{3}$. It induces the fluctuation fields around the "slowly moving" vortex background. Up to first order in the derivatives $\partial_{\alpha}\left(x_{\alpha}=t, x_{3}\right)$, the scalar field $Q$ and $A_{\bar{z}}$ are not modified

$$
\begin{aligned}
Q & =U\left(x_{\alpha}\right)^{\dagger} Q_{0} U\left(x_{\alpha}\right)+\mathcal{O}\left(\partial_{\alpha}^{2}\right), \\
A_{\bar{z}} & =U\left(x_{\alpha}\right)^{\dagger} A_{0 \bar{z}} U\left(x_{\alpha}\right)+\mathcal{O}\left(\partial_{\alpha}^{2}\right),
\end{aligned}
$$

\footnotetext{
${ }^{4}$ The square root of the matrices $X$ and $Y$ always exists. For $G=S O(2 n)$ and $U S p(2 n)$, it can be written as $X^{\frac{1}{2}}=U_{X}\left(1+|D|^{2}\right)^{\frac{1}{2}} U_{X}^{\dagger}$ and $Y^{\frac{1}{2}}=U_{Y}\left(1+|D|^{2}\right)^{\frac{1}{2}} U_{Y}^{\dagger}$ where $U_{X}$ and $U_{Y}$ are unitary matrices and $D$ is a diagonal matrix such that $B=U_{Y} D U_{X}^{\dagger}$.
} 
where $Q_{0}$ and $A_{0 \bar{z}}$ are the static BPS configurations. For notational simplicity, we use the following singular gauge fixing

$$
Q_{0}=v \exp \left[\left(\log |z|-\frac{1}{2} \psi\right) \lambda_{h}\right], \quad A_{0 \bar{z}}=-\frac{i}{2}\left(\partial_{\bar{z}} \psi-\frac{1}{\bar{z}}\right) \lambda_{h} .
$$

The fluctuations of the gauge fields along the vortex worldsheet $A_{\alpha}$ can be determined by solving the equations of motion

$$
\frac{2}{g^{2}} \mathcal{D}^{\mu} F_{\mu \alpha}=i\left[\left(\mathcal{D}_{\alpha} Q\right) Q^{\dagger}-Q\left(\mathcal{D}_{\alpha} Q^{\dagger}\right)\right]
$$

In the slowly moving background, the solution is given by 5

$$
A_{\alpha}=-i U^{\dagger} \partial_{\alpha} U+i U^{\dagger}\left[Q_{0}\left(\delta_{\alpha}^{\dagger} U\right) U^{\dagger} Q_{0}^{-1}-\text { h.c. }\right] U+\mathcal{O}\left(\partial_{\alpha}^{3}\right)
$$

where we have decomposed the derivative $\partial_{\alpha}$ into the holomorphic and anti-holomorphic parts as

$$
\partial_{\alpha}=\delta_{\alpha}+\delta_{\alpha}^{\dagger}, \quad \delta_{\alpha}=\partial_{\alpha} b^{i} \frac{\partial}{\partial b^{i}}, \quad \delta_{\alpha}^{\dagger}=\partial_{\alpha} \bar{b}^{\bar{i}} \frac{\partial}{\partial \bar{b}^{i}}
$$

Although $Q_{0}^{-1}$ is singular at the vortex center, we can check that the gauge field $A_{\alpha}$ is non-singular by using the explicit form of $U$ given in Eq. (2.35)

$$
A_{\alpha}=i\left(1-|z| e^{-\frac{1}{2} \psi}\right) U^{\dagger}\left(\begin{array}{c|c}
0 & X^{-\frac{1}{2}} \partial_{\alpha} B^{\dagger} Y^{-\frac{1}{2}} \\
\hline-Y^{-\frac{1}{2}} \partial_{\alpha} B X^{-\frac{1}{2}} & 0
\end{array}\right) U
$$

where we have used $\delta_{\alpha} B=\partial_{\alpha} B$ and $\delta_{\alpha} B^{\dagger}=\partial_{\alpha} B^{\dagger}$. Substituting the scalar field $Q$ and gauge field $A_{\mu}$ into the bulk action and integrating over the $\left(x_{1}, x_{2}\right)$-plane, we obtain the effective action of the form

$$
S_{\mathrm{eff}}=\int d t d x_{3}\left(-T_{v}+g_{i \bar{j}} \partial_{\alpha} b^{i} \partial^{\alpha} \bar{b}^{\bar{j}}\right)
$$

where the first term is the tension of the vortex string and $g_{i \bar{j}}$ is the metric of the orientational moduli space, which is given in terms of the matrices $g_{l}$ and $g_{u}$ by

$$
g_{i \bar{j}}=\frac{4 \pi}{g^{2}} \frac{\partial}{\partial \bar{b}^{j}} \operatorname{Tr}\left[\left(g_{l} \frac{\partial}{\partial b^{i}} g_{l}^{-1}\right) g_{u}^{-1} \lambda_{h} g_{u}\right] .
$$

If we use the matrix $B$, the effective action on the vortex worldsheet takes the form [9]

$$
S_{\mathrm{eff}}=\frac{4 \pi}{g^{2}} \int d t d x_{3} \operatorname{Tr}\left[\left(\mathbf{1}_{q}+B B^{\dagger}\right)^{-1} \partial_{\alpha} B\left(\mathbf{1}_{p}+B^{\dagger} B\right)^{-1} \partial^{\alpha} B^{\dagger}\right]
$$

where we have ignored the string tension $T_{v}$.

\footnotetext{
${ }^{5}$ A general formula for the solution can be obtained by using the moduli matrix approach, see [33, 20] for the $U(N)$ case.
} 


\subsection{Mass deformation}

So far we have discussed the orientational moduli and their effective action in the massless theory. Next let us discuss the effect of the bulk mass term on the effective action of the vortex string.

The mass matrix $M$ is taken to be in the Cartan subalgebra of $G$

$$
M=\mathbf{m} \cdot \mathbf{H}, \quad \mathbf{m}=\left(m_{1}, m_{2}, \cdots, m_{r}\right) .
$$

If $\mathbf{m}$ is a generic vector, all the mass elements are different and the color-flavor symmetry is broken to the product of the Cartan subgroups. Then, the F-term condition or Eq. (2.7) requires

$$
[\mathbf{m} \cdot \mathbf{H}, Q]=0
$$

Therefore, the magnetic flux is forced to be oriented in the particular directions specified by the weight vectors $\tilde{\boldsymbol{\nu}}$. Note that these solutions are invariant under the Cartan subgroups.

The mass deformation breaks the color-flavor symmetry, and the remaining global symmetry of the system depends on the mass matrix. We will however assume that $\Lambda_{\mathrm{NLSM}} \ll m_{i} \ll g v$, where $\Lambda_{\mathrm{NLSM}}$ is the intrinsic scale of the sigma model while $g v$ is the mass of the particles in the bulk theory. This condition allows us to treat the deformation as a shallow potential on the string worldsheet. In general we shall take all the elements in the mass matrix to be distinct; when some of them coincide, a non-Abelian subgroup of the color-flavor group will emerge, giving non-Abelian moduli to kinks [34] and monopoles [35].

The potential on the moduli space induced by the mass term can be calculated in a way similar to the kinetic term of the effective action (2.45) discussed in the previous section. First let us determine the modification of the fields perturbatively in terms of the mass parameter. Up to first order in the masses, the background vortex configuration is not modified. The adjoint scalar field $\Phi$ can be determined by solving the equation of motion in the vortex background

$$
\frac{2}{g^{2}} \mathcal{D}_{\mu} \mathcal{D}^{\mu} \Phi=-(\Phi Q-Q M) Q^{\dagger}-Q\left(Q^{\dagger} \Phi-M Q^{\dagger}\right)
$$

Since $\Phi$ is of order $m_{i}, \mathcal{D}_{\alpha} \mathcal{D}^{\alpha} \Phi$ is of order $\mathcal{O}\left(m \partial_{\alpha}^{2}\right)$, which is small. We obtain the following solution to the equation of motion

$$
\Phi=M+i U^{\dagger}\left(\delta_{m}+\delta_{m}^{\dagger}\right) U-i U^{\dagger}\left[Q_{0}\left(\delta_{m}^{\dagger} U\right) U^{\dagger} Q_{0}^{-1}-\text { h.c. }\right] U+\mathcal{O}\left(m^{3}, m \partial_{\alpha}^{2}\right)
$$

where we have defined the derivative operators 6

$$
\delta_{m}=k^{i} \frac{\partial}{\partial b^{i}}, \quad \delta_{m}^{\dagger}=\bar{k}^{\bar{i}} \frac{\partial}{\partial \bar{b}^{i}}, \quad k^{i}=i\left(\boldsymbol{\alpha}_{i} \cdot \mathbf{m}\right) b^{i}, \quad \bar{k}^{\bar{i}}=-i\left(\boldsymbol{\alpha}_{\bar{i}} \cdot \mathbf{m}\right) \bar{b}^{\bar{i}} .
$$

\footnotetext{
${ }^{6}$ There is no summation on $i$ in $k^{i}=i\left(\boldsymbol{\alpha}_{i} \cdot \mathbf{m}\right) b^{i}$, and $\bar{k}^{\bar{i}}=-i\left(\boldsymbol{\alpha}_{\bar{i}} \cdot \mathbf{m}\right) \bar{b}^{\bar{i}}$.
} 
By using the explicit form of the matrix $U$, we obtain

$$
\Phi=M-i\left(1-|z| e^{-\frac{1}{2} \psi}\right) U^{\dagger}\left(\begin{array}{c|c}
0 & X^{-\frac{1}{2}}\left(\delta_{m}^{\dagger} B^{\dagger}\right) Y^{-\frac{1}{2}} \\
\hline-Y^{-\frac{1}{2}}\left(\delta_{m} B\right) X^{-\frac{1}{2}} & 0
\end{array}\right) U
$$

Note that $k^{i}$ can be interpreted as the holomorphic Killing vector on the moduli space. The corresponding isometry, which we call $U(1)_{M}$, is a subgroup of $G$ and acts on the matrix $U$ and the complex coordinates $b^{i}$ as

$$
U \rightarrow e^{i M \vartheta} U e^{-i M \vartheta} \quad \Longleftrightarrow \quad b^{i} \rightarrow e^{i\left(\boldsymbol{\alpha}_{i} \cdot \mathbf{m}\right) \vartheta} b^{i} \quad\left(\text { Def. } U(1)_{M}\right)
$$

Then substituting the configuration into the bulk action and integrating over the $\left(x_{1}, x_{2}\right)$-plane, we obtain the potential of the form [36, 37]

$$
V_{\text {eff }}=g_{i \bar{j}} k^{i} \bar{k}^{\bar{j}}
$$

This is the squared norm of the Killing vector and has minima at the zeros of $k^{i}$, viz. the fixed points of the $U(1)_{M}$ isometry. This implies that the vortex in the massive theory has minimum energy if it is invariant under $U(1)_{M} \subset G_{C+F}$.

The potential of this form can also be obtained in the following way. First let us consider the massless sigma model with one additional compact direction $\vartheta$ which has period $2 \pi R$

$$
S_{\text {eff }}=\frac{1}{2 \pi R} \int d t d x_{3} d \vartheta g_{i \bar{j}}\left(\partial_{\alpha} b^{i} \partial^{\alpha} \bar{b}^{\bar{j}}-\partial_{\vartheta} b^{i} \partial_{\vartheta} \bar{b}^{\bar{j}}\right)
$$

Then we impose the following twisted boundary condition with respect to the $U(1)_{M}$ symmetry

$$
b^{i}(\vartheta+2 \pi R)=e^{2 \pi i R\left(\boldsymbol{\alpha}_{i} \cdot \mathbf{m}\right)} b^{i}(\vartheta) .
$$

where there is no summation on $i$. If we ignore the infinite tower of the Kaluza-Klein modes, the $\vartheta$-dependence of the field can be determined as

$$
b^{i}\left(t, x_{3}, \vartheta\right)=e^{i\left(\boldsymbol{\alpha}_{i} \cdot \mathbf{m}\right) \vartheta} b^{i}\left(t, x_{3}\right)
$$

Substituting this lowest mode into the massless effective action (2.54), we obtain the same potential term as that of Eq. (2.53)

$$
S_{\mathrm{eff}}=\int d t d x_{3} g_{i \bar{j}}\left(\partial_{\alpha} b^{i} \partial^{\alpha} \bar{b}^{\bar{j}}-k^{i} \bar{k}^{\bar{j}}\right) .
$$

In the next section, we will use this method to find the explicit potentials on the vortex worldsheet for $G=S U(N), S O(2 n)$ and $U S p(2 n)$.

\footnotetext{
${ }^{7} \mathrm{~A}$ similar result has been obtained for $1 / 4$ BPS monopoles in $\mathcal{N}=4$ theories in Ref. [38.
} 
In the $G=S U(N)$ case, the generic mass matrix breaks the gauge symmetry to $U(1)^{N-1}$ and one observes that the breaking gives rise to monopoles. Turning on the FI parameter $\xi$, the monopoles are still there but will be confined to live on the vortex string. They turn out to be kinks along the vortex [3, 5, 8]. We will study the generalization of this phenomenon for the $G=S O(2 n)$ and $U S p(2 n)$ theories in section 4 .

\section{Mass-deformed sigma model}

\section{$3.1 \mathbb{C} P^{N-1}$ as a warm up}

Let us take a simple example to illustrate the method. In the case of $G=S U(2)$, the orientational moduli space is the complex projective space $\mathbb{C} P^{1} \cong S U(2) / U(1)$. The Kähler potential for the $\mathbb{C} P^{1}$ model is

$$
K=\frac{4 \pi}{g^{2}} \log \left(1+|b|^{2}\right)
$$

giving rise to the sigma model

$$
\mathcal{L}=\frac{4 \pi}{g^{2}} \frac{\left|\partial_{\alpha} b\right|^{2}}{\left(1+|b|^{2}\right)^{2}}
$$

where $b \in \mathbb{C}$ is the inhomogeneous coordinate on $\mathbb{C} P^{1}$. Now let us use the method of Ref. [7] to generate the twisted mass potential on the vortex worldsheet induced by the bulk mass term

$$
M=2\left(\begin{array}{cc}
m & 0 \\
0 & -m
\end{array}\right) .
$$

First we have to use the $U(1)_{M}$ global symmetry of the system at hand. In the case of the $\mathbb{C} P^{1}$ model, the $U(1)_{M}$ symmetry acts on the coordinate $b$ as a global phase rotation

$$
b \rightarrow e^{-i 2 m \vartheta} b
$$

which leaves the Lagrangian (3.2) invariant. Taking advantage of an argument similar to that of the last section, we obtain

$$
b(t, z, \vartheta)=e^{-i 2 m \vartheta} b(t, z)
$$

Plugging this field back into the Lagrangian (3.2) leaves us with the following mass-deformed theory [39], 6]

$$
\mathcal{L}=\frac{4 \pi}{g^{2}} \frac{\left|\partial_{\alpha} b\right|^{2}-4 m^{2}|b|^{2}}{\left(1+|b|^{2}\right)^{2}}
$$


The theory is known to have two vacua. The description we have used above uses the inhomogeneous coordinates on $\mathbb{C} P^{1}$ and hence we need two patches to describe the theory. On each patch the vacuum is seen to be given by $b=0$, which corresponds to $b \rightarrow \infty$ on the other patch. As we have seen in the previous section, the potential is the squared norm of the Killing vector of $U(1)_{M}$. Indeed the vacua $b=0$ and $b \rightarrow \infty$ are the fixed points of the $U(1)_{M}$ symmetry. Hence we have checked (trivially) that the number of vacua found is indeed two, in accord with the literature.

Generalizing the above discussion to the case of $G=S U(N)$, we have the following Kähler potential for the sigma model on $\mathbb{C} P^{N-1}$

$$
K=\frac{4 \pi}{g^{2}} \log \left(1+b^{\dagger} b\right)
$$

where $b$ is an $(N-1)$-component complex column vector. The Lagrangian reads [40, 1, 8]

$$
\mathcal{L}=\frac{4 \pi}{g^{2}}\left[\frac{\partial_{\alpha} b^{\dagger} \partial^{\alpha} b}{1+b^{\dagger} b}-\frac{\left(b^{\dagger} \partial_{\alpha} b\right)\left(\partial^{\alpha} b^{\dagger} b\right)}{\left(1+b^{\dagger} b\right)^{2}}\right] .
$$

The $U(1)_{M}$ global symmetry of the Lagrangian is expressed as

$$
b \rightarrow \exp \left(-i m_{1} \vartheta\right) \exp \left(i M_{N-1} \vartheta\right) b
$$

where we have assumed that the bulk mass is

$$
M=\left(\begin{array}{l|l}
m_{1} & \\
\hline & M_{N-1}
\end{array}\right) .
$$

Keeping only the lowest mode

$$
b(t, z, \vartheta)=e^{i M_{0} \vartheta} b_{0}(t, z),
$$

where $M_{0}=-m_{1} \mathbf{1}_{N-1}+M_{N-1}$. Insertion of this field into the Lagrangian and dropping the suffix of $b$ gives us the deformed sigma model

$$
\mathcal{L}=\frac{4 \pi}{g^{2}}\left[\frac{\partial_{\alpha} b^{\dagger} \partial^{\alpha} b-b^{\dagger} M_{0}^{2} b}{1+b^{\dagger} b}-\frac{\left(b^{\dagger} \partial_{\alpha} b\right)\left(\partial^{\alpha} b^{\dagger} b\right)-\left(b^{\dagger} M_{0} b\right)^{2}}{\left(1+b^{\dagger} b\right)^{2}}\right] .
$$

A vacuum satisfying the fixed point condition $M_{0} b=0$ is just the origin of this coordinate patch. Hence, there will be $N$ vacua corresponding to $N$ patches covering $\mathbb{C} P^{N-1}$,

$$
n_{\mathrm{vacua}}^{S U(N)}=N
$$




\subsection{The $S O(2 n) / U(n)$ and $U S p(2 n) / U(n)$ sigma models}

Let us now apply the aforementioned technique to the $S O(2 n) / U(n)$ and $U S p(2 n) / U(n)$ sigma models on the vortex worldsheet. We will treat them on the same footing in the following. For $S O(2 n) / U(n)$ the field $B^{\mathrm{T}}=-B$ is an anti-symmetric matrix valued field while for $U S p(2 n) / U(n)$ it is symmetric $B^{\mathrm{T}}=B$. The Kähler potential

$$
K=\frac{4 \pi}{g^{2}} \operatorname{Tr} \log \left(\mathbf{1}_{n}+B B^{\dagger}\right)
$$

gives rise to the Lagrangian which we found in the previous section, i.e.,

$$
\mathcal{L}=\frac{4 \pi}{g^{2}} \operatorname{Tr}\left\{\left(\mathbf{1}_{n}+B^{\dagger} B\right)^{-1} \partial_{\alpha} B^{\dagger}\left(\mathbf{1}_{n}+B B^{\dagger}\right)^{-1} \partial^{\alpha} B\right\} .
$$

Since we have assumed that $M$ is in the Cartan subalgebra of $G$, the mass matrix takes the form

$$
M=\left(\begin{array}{l|l}
M_{n} & \\
\hline & -M_{n}
\end{array}\right), \quad M_{n}=\operatorname{diag}\left(m_{1}, m_{2}, \cdots, m_{n}\right) .
$$

The $U(1)_{M}$ action on $B$ can be seen from $U \rightarrow e^{-i \vartheta M} U e^{i \vartheta M}$ to be

$$
B \rightarrow e^{i M_{n} \vartheta} B e^{i M_{n} \vartheta}
$$

As above, we expand the field in modes and keep just the lowest mode giving rise to

$$
B(t, z, \vartheta)=e^{i M_{n} \vartheta} B_{0}(t, z) e^{i M_{n} \vartheta}
$$

Upon inserting this field in the Lagrangian (3.15) and dropping the suffix, we obtain the following mass-deformed sigma model

$$
\begin{aligned}
\mathcal{L}=\frac{4 \pi}{g^{2}} \operatorname{Tr} & \left\{\left(\mathbf{1}_{n}+B^{\dagger} B\right)^{-1} \partial_{\alpha} B^{\dagger}\left(\mathbf{1}_{n}+B B^{\dagger}\right)^{-1} \partial^{\alpha} B\right. \\
& \left.-\left(\mathbf{1}_{n}+B^{\dagger} B\right)^{-1}\left\{M_{n}, B^{\dagger}\right\}\left(\mathbf{1}_{n}+B B^{\dagger}\right)^{-1}\left\{M_{n}, B\right\}\right\} .
\end{aligned}
$$

As the mass matrix is Hermitian, the vacuum equation reads

$$
\left\{M_{n}, B\right\}=0
$$

which in general can only be satisfied for $B=0.8$ However, there exist other vacua in the coordinate patches which are not covered by $B$. In the next section we will see that the numbers of vacua of the $S O(2 n) / U(n)$ and $U S p(2 n) / U(n)$ sigma models are

$$
n_{\text {vacua }}^{S O(2 n)}=2^{n-1}, \quad n_{\text {vacua }}^{U S p(2 n)}=2^{n},
$$

\footnotetext{
${ }^{8}$ We assume that $m_{i} \neq \pm m_{j}$ to break the color-flavor group to Cartan generators.
} 


\begin{tabular}{l||c} 
moduli space $\mathcal{M}_{\text {orientation }}$ & $\chi\left(\mathcal{M}_{\text {orientation }}\right)$ \\
\hline \hline$\frac{S O(2 n)}{U(n)}$ & $2^{n-1}$ \\
$\frac{U S p(2 n)}{U(n)}$ & $2^{n}$ \\
$\mathbb{C} P^{N-1}=\frac{S U(N)}{S U(N-1) \times U(1)}$ & $N$ \\
$G r_{N, k}=\frac{S U(N)}{S(U(k) \times U(N-k))}$ & $\left(\begin{array}{l}N \\
k\end{array}\right)$ \\
$Q^{2 n-2}=\frac{S O(2 n)}{S O(2) \times S O(2 n-2)}$ & $2 n$
\end{tabular}

Table 1: The Euler characteristics of various orientational moduli spaces.

respectively. Although our analysis here is limited to the worldsheet action of the single winding vortex $(k=1)$, we can generalize the discussion to higher winding cases. In the case of $k \geq 2$, we have various choices for the representation of the coweight $\tilde{\boldsymbol{\nu}}$, which determines the orbit of the $G_{C+F}$ symmetry. As an example, the mass-deformed sigma model on the quadric surface $Q^{2 n-2}$ $(G=S O(2 n), k=2, \tilde{\boldsymbol{\nu}}=$ vector representation) is discussed in Appendix D.

We have now considered a few worldsheet sigma models which are all low-energy effective descriptions of non-Abelian vortex systems. For the sigma models we are considering, the number of vacua in the classical regime is equal to the Euler number of the target space $\chi\left(\mathcal{M}_{\text {orientation }}\right)$ [9], see Table 1. This is consistent with the Witten index [41] and expected to remain the same in the quantum regime. In the next section we will consider the kinks interpolating the different vacua of the vortex worldsheet theory, viz. the sigma model.

\section{Monopoles as kinks on the vortex}

In this section we discuss kink configurations in the effective action on the worldsheet of the $U(1) \times S O(2 n)$ and $U(1) \times U S p(2 n)$ vortices. We will see that the kinks on the vortex worldsheet can be interpreted as the $S O(2 n)$ and $U S p(2 n)$ monopoles from the viewpoint of the bulk theory.

\section{1 $S U(2)$ monopole}

For illustration, let us first review the $1 / 4$ BPS configuration of the kink monopole in the $G=$ $S U(2)$ case [3].

The orientational moduli of the $U(2)$ vortex is $\mathbb{C} P^{1} \cong S U(2) / U(1)$. In the presence of the mass term $M=m \sigma_{3}$, the potential is induced and only two configurations are left to be the minimal energy configurations (which we call the vortex vacua). At these points, the magnetic 
flux $\lambda=-\frac{1}{2 \pi} \int d x_{1} d x_{2} F_{12}$ is given by

$$
\lambda_{\text {highest }}=\left(\begin{array}{ll}
1 & \\
& 0
\end{array}\right), \quad \lambda_{\text {lowest }}=\left(\begin{array}{ll}
0 & \\
& 1
\end{array}\right) .
$$

The 1/4 BPS equations (2.6)-(2.8) do admit configurations of vortices which approach the vortex vacua at $x_{3} \rightarrow \pm \infty$ as

$$
\lim _{x_{3} \rightarrow-\infty} \lambda=\lambda_{\text {highest }}, \quad \lim _{x_{3} \rightarrow \infty} \lambda=\lambda_{\text {lowest }} .
$$

For such a configuration, the energy (2.10) is given by the difference between the magnetic fluxes at $x_{3} \rightarrow \pm \infty$, i.e. the magnetic charge inside the vortex

$$
E=-\frac{4 \pi}{g^{2}} \int d x_{3} \partial_{x_{3}} \operatorname{Tr}[\lambda \Phi]+\int d x_{3} T_{v}=\frac{8 \pi m}{g^{2}}+\int d x_{3} T_{v}
$$

where the second term represents the vortex tension. Note that the adjoint scalar $\Phi$ approaches the $\operatorname{VEV}\langle\Phi\rangle=M$ at spatial infinity

$$
\lim _{x_{3} \rightarrow \pm \infty} \Phi=m \sigma_{3}
$$

The energy of Eq. (4.3) is given by the mass of the monopole and the vortex tension. Hence we can interpret this as the energy of the 1/4 BPS configuration of the confined monopole attached to two vortices having magnetic flux $\lambda_{\text {highest }}$ and $\lambda_{\text {lowest }}$, respectively (see Fig.11). Fig.1 is the first full numerical solution of a confined monopole.

We can discuss the same configuration using the worldsheet effective action of the vortex. Using the effective action (3.6) for the $U(2)$ vortex in the massive theory, we can easily find the BPS equation for the kink by rewriting the energy of the static configuration as follows

$$
E=\int d x_{3} \frac{4 \pi}{g^{2}} \frac{\left|\partial_{x_{3}} b-2 m b\right|^{2}}{\left(1+|b|^{2}\right)^{2}}+\int d x_{3} \frac{4 \pi}{g^{2}} \partial_{x_{3}} \sigma \geq \frac{4 \pi}{g^{2}}[\sigma(\infty)-\sigma(-\infty)],
$$

where $\sigma$ is the standard height function of $\mathbb{C} P^{1}$ which is given by

$$
\sigma=-m \frac{1-|b|^{2}}{1+|b|^{2}} .
$$

The BPS equation and its solution are given by

$$
\partial_{x_{3}} b-2 m b=0 \quad \Longrightarrow \quad b\left(x_{3}\right)=b_{0} e^{2 m x_{3}}
$$

where $b_{0}$ is a complex constant which corresponds to the position and phase moduli of the kink. Although the solution $b \rightarrow \infty$ at $x_{3} \rightarrow \infty$, this is an artifact of the choice of the coordinate. The moduli field $b$ is the inhomogeneous coordinate on $\mathbb{C} P^{1}$. In order to see the other vacuum, one 


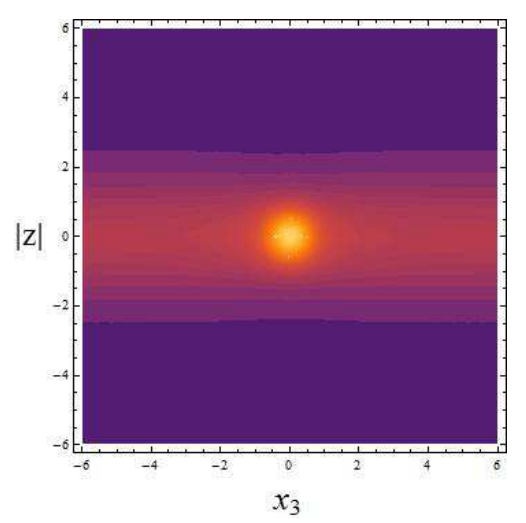

(a) energy density
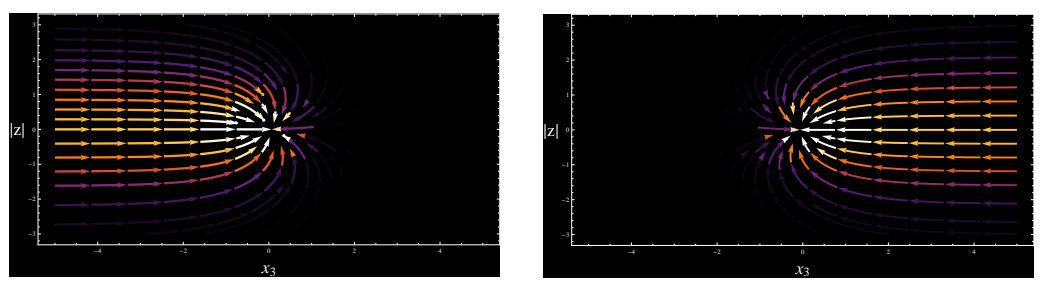

(b) $\operatorname{Tr}\left[B_{i}\left(\mathbf{1}+\sigma_{3}\right)\right]$

(c) $\operatorname{Tr}\left[B_{i}\left(\mathbf{1}-\sigma_{3}\right)\right]$

Fig. 1: (a) The energy density profile of the vortex-monopole configuration (numerical solution of the $1 / 4$ BPS equations). The energy is localized along the vortex $\left(|z|=\left|x_{1}+i x_{2}\right|=0\right)$ and around the monopole $\left(|z|=x_{3}=0\right)$. (b, c) The magnetic flux projected onto $\mathbf{1}+\sigma_{3} \propto \lambda_{\text {highest }}$ and $\mathbf{1}-\sigma_{3}=\lambda_{\text {lowest }}$. The monopole is attached to two vortices with magnetic flux $\lambda_{\text {highest }}$ and $\lambda_{\text {lowest }}$, respectively. The plots for negative $|z|$ are simply mirror images in order to illustrate the cross section of the configuration.

needs to change the patch, say $b^{\prime}=1 / b(b \neq 0)$. This BPS configuration saturates the bound (4.5) and the kink mass is given by

$$
E=\frac{4 \pi}{g^{2}}[\sigma(\infty)-\sigma(-\infty)]=\frac{8 \pi m}{g^{2}}
$$

This kink mass is in precise agreement with the monopole mass. In this way, we can identify the kink interpolating between the distinct vortex vacua with the monopole confined inside the vortex [3].

\section{2 $S O(2 n)$ and $U S p(2 n)$ monopoles}

\subsubsection{BPS kink equations}

We will use the following convention for the Cartan generators

$$
\mathbf{H}=\left(\begin{array}{l|l}
\mathbf{H}_{n} & \\
\hline & -\mathbf{H}_{n}
\end{array}\right), \quad \mathbf{H}_{n}=\left(\begin{array}{lll}
\boldsymbol{e}_{1} & & \\
& \ddots & \\
& & \boldsymbol{e}_{n}
\end{array}\right),
$$


where $\left\{\boldsymbol{e}_{i}\right\}$ are the standard orthonormal basis. With this normalization, the highest weight vector of the (Weyl) spinor representation of $S O(2 n)$ and $S O(2 n+1)$ is given by

$$
\tilde{\boldsymbol{\nu}}_{h}=\frac{1}{2}\left(\boldsymbol{e}_{1}+\cdots+\boldsymbol{e}_{n}\right) .
$$

The mass matrix takes the form

$$
M=\mathbf{m} \cdot \mathbf{H}=\left(\begin{array}{l|l}
M_{n} & \\
\hline & -M_{n}
\end{array}\right) .
$$

Without loss of generality, we can always choose the ordering of the masses by using the $S O(2 n)$ and $U S p(2 n)$ rotations as

$$
\mathbf{m}=\left(m_{1}, m_{2}, \cdots, m_{n}\right), \quad m_{1} \geq m_{2} \geq \cdots \geq m_{n} \geq 0
$$

If some of the masses are equal, a non-Abelian subgroup of $G_{C+F}$ is unbroken, while the symmetry is maximally broken to $U(1)^{n}$ for a non-degenerate mass matrix. For simplicity, we restrict ourselves to the case of maximal symmetry breaking $m_{1}>m_{2}>\cdots>m_{n}>0$, which implies that

$$
\mathbf{m} \cdot \boldsymbol{\alpha}_{i}>0
$$

for all positive root vectors $\boldsymbol{\alpha}_{i}$. In other words, $\mathbf{m}$ is a vector in the interior of the positive Weyl chamber.

The time-independent energy density of the effective sigma model can be decomposed into a positive semi-definite term and a total derivative term

$$
\mathcal{E}=\frac{4 \pi}{g^{2}} \operatorname{Tr}\left[X^{-1}\left(\partial_{x_{3}} B^{\dagger}-\left\{M_{n}, B^{\dagger}\right\}\right) Y^{-1}\left(\partial_{x_{3}} B-\left\{M_{n}, B\right\}\right)\right]+\frac{4 \pi}{g^{2}} \partial_{x_{3}} \sigma,
$$

where $X$ and $Y$ are the matrices given in Eq. (2.34). The function $\sigma$, which is called the moment map of the $U(1)_{M}$ action, is given by

$$
\sigma=\operatorname{Tr}\left[M_{n}-X^{-1} M_{n}-Y^{-1} M_{n}\right]
$$

Note that $\sigma$ satisfies

$$
\partial_{x_{3}} \sigma=\operatorname{Tr}\left[X^{-1} \partial_{x_{3}} B^{\dagger} Y^{-1}\left\{M_{n}, B\right\}+X^{-1}\left\{M_{n}, B^{\dagger}\right\} Y^{-1} \partial_{x_{3}} B\right] .
$$

The first term in the energy density (4.14) is positive semi-definite and vanishes if the matrix $B$ satisfies

$$
\partial_{x_{3}} B=\left\{M_{n}, B\right\} .
$$


This is the BPS equation for the kinks on the vortex worldsheet. If the matrix $B$ satisfies the BPS equation, the energy of the configuration, which can be interpreted as the total mass of the BPS kinks, is given by the boundary values of the function $\sigma$

$$
M_{\mathrm{kink}}=\frac{4 \pi}{g^{2}} \int_{-\infty}^{\infty} d x_{3} \partial_{x_{3}} \sigma=\frac{4 \pi}{g^{2}}[\sigma(\infty)-\sigma(-\infty)]
$$

The physical meaning of the function $\sigma$ can be seen by using Eqs. (2.20), (2.49) and (2.35)

$$
\frac{1}{2 \pi} \int d x_{1} d x_{2} \operatorname{Tr}\left[F_{12} \Phi\right]=-\operatorname{Tr}\left[U^{\dagger} \lambda_{h} U M\right]=\sigma
$$

This implies that the function $\sigma$ is the magnetic flux of the vortex projected onto the internal direction specified by the adjoint scalar $\Phi$. The total mass (4.18) is proportional to the difference of the magnetic flux, i.e. the magnetic charge inside the vortex string

$$
M_{\mathrm{kink}}=\frac{4 \pi}{g^{2}} \mathbf{m} \cdot \mathbf{g},
$$

where we have defined the magnetic charge vector $\mathbf{g}$ as

$$
\begin{aligned}
\mathbf{g} & \equiv \int d x_{3} \operatorname{Tr}\left[X^{-1} \partial_{x_{3}} B^{\dagger} Y^{-1}\left\{\mathbf{H}_{n}, B\right\}+X^{-1}\left\{\mathbf{H}_{n}, B^{\dagger}\right\} Y^{-1} \partial_{x_{3}} B\right] \\
& =\int d x_{3} \partial_{x_{3}} \operatorname{Tr}\left[\mathbf{H}_{n}-X^{-1} \mathbf{H}_{n}-Y^{-1} \mathbf{H}_{n}\right] .
\end{aligned}
$$

Therefore the kinks on the vortex worldsheet are magnetically charged objects, i.e. they are the magnetic monopoles.

The general solution to the BPS equation (4.17) can be easily obtained as

$$
B=e^{M_{n} x_{3}} B_{0} e^{M_{n} x_{3}},
$$

where the matrix elements of $B_{0}$ are the integration constants, namely the moduli parameters of the BPS configurations. Although the solution (4.22) diverges at spatial infinity $x_{3} \rightarrow \infty$, this is an artifact of the choice of the coordinates as in the example of the $S U(2)$ monopole.

In order to see the configuration at $x_{3} \rightarrow \infty$, we need to change the patch specified by $B$ to other patches. To this end, let us use the fact that the target space $S O(2 n) / U(n)$ and $U S p(2 n) / U(n)$ can be embedded into the complex Grassmannian $\operatorname{Gr}(2 n, n)$. As is well known, the complex Grassmannian can be described by an $n$-by- $2 n$ matrix $\Lambda$ with the following equivalence relation

$$
\Lambda \sim V \Lambda, \quad V \in G L(n, \mathbb{C})
$$


The coset spaces $S O(2 n) / U(n)$ and $U S p(2 n) / U(n)$ are subspaces in the complex Grassmannian $\operatorname{Gr}(2 n, n)$ defined by the following constraints on the matrix $\Lambda$ [42]

$$
\Lambda J \Lambda^{\mathrm{T}}=0, \quad J=\left(\begin{array}{c|c}
\mathbf{0} & \mathbf{1}_{n} \\
\hline \epsilon \mathbf{1}_{n} & \mathbf{0}
\end{array}\right),
$$

where $\epsilon=1$ for $S O(2 n)$ and $\epsilon=-1$ for $U S p(2 n)$. Since the group $G$ transitively acts on the coset space, any point on the coset space can be obtained from a specific matrix $\Lambda$ by the $G$ transformations

$$
\Lambda \rightarrow \Lambda U, \quad U \in G
$$

Let us take the base point at $B=0$ as the corresponding matrix

$$
\Lambda_{h} \equiv\left(\begin{array}{c|c}
\mathbf{0}_{n} & \left.\mathbf{1}_{n}\right)
\end{array}\right.
$$

where the suffix of $\Lambda_{h}$ stands for the vortex configuration (2.17)-(2.18) which is associated with the highest weight vector $\tilde{\boldsymbol{\nu}}_{h}$. Then, any point on the coset space is given by

$$
\Lambda=\Lambda_{h} U, \quad U \in G
$$

Note that the action of the subgroup $H \cong U(n)$ is trivial on $\Lambda_{h}$, so that the whole set of matrices $\Lambda$ is identified with the coset space $G / H$. If we multiply by the group element $U \in G$ given in Eq. (2.35), the matrix $\Lambda$ takes the form

$$
\Lambda=\Lambda_{h} g_{u} g_{l} \sim \Lambda_{h} g_{l}=\left(B \mid \mathbf{1}_{n}\right)
$$

This shows that the element of the parabolic subgroup $g_{u} \in P$ trivially acts on $\Lambda_{h}$, while the action of the complexified group element $g_{l} \in G^{\mathbb{C}}$, generated by the lowering operators $E_{-\boldsymbol{\alpha}}$ with $\tilde{\boldsymbol{\nu}}_{h} \cdot \boldsymbol{\alpha}>0$, is non-trivial.

In the following, we discuss the vacua and kink configurations in the $S O(2 n) / U(n)$ and $U S p(2 n) / U(n)$ sigma models in terms of the $n$-by- $2 n$ matrix $\Lambda$. Since these sigma models can be regarded as the low energy effective theories of a $U(n)$ gauge theory with $2 n$ flavors obeying the F-term constraint (4.24), the discussion below can be viewed as a generalization of the construction of the domain walls in the $U(n)$ gauge theory (or the Grassmann sigma model) discussed in Ref. [17, 18, 43, 44].

\footnotetext{
${ }^{9}$ In the case of $S O(2 n) / U(n)$, the set of the solutions to the constraint (4.24) consists of two disjoint copies of $S O(2 n) / U(n)$, one of which can be identified with the moduli space of vortices of definite chirality.
} 


\subsubsection{Vacua on the vortex worldsheet}

Now let us discuss the vacuum configurations in terms of the matrix $\Lambda$. The vacuum condition $\left\{M_{n}, B\right\}=0$ implies that the vacua are fixed points of the $U(1)_{M}$ transformation

$$
B \rightarrow e^{i M_{n} \vartheta} B e^{i M_{n} \vartheta}=B
$$

In terms of the matrix $\Lambda$, this condition of the fixed points can be rewritten as

$$
\Lambda \rightarrow \Lambda e^{i M \vartheta} \sim \Lambda
$$

We can easily show that $\Lambda_{h}$ is one of the fixed points of $U(1)_{M}$

$$
\Lambda_{h} e^{i M \vartheta}=e^{-i M_{n} \vartheta} \Lambda_{h} \sim \Lambda_{h}
$$

The other matrices corresponding to fixed points can be found by multiplying matrix $\Lambda_{h}$ by Weyl group transformations of $G$ as follows

$$
\Lambda=\Lambda_{h} w, \quad w=w_{\boldsymbol{\alpha}_{i}} w_{\boldsymbol{\alpha}_{j}} \cdots w_{\boldsymbol{\alpha}_{k}} \in G
$$

where $w_{\boldsymbol{\alpha}_{i}}$ are the generators of the Weyl group corresponding to reflections with respect to the simple roots $\boldsymbol{\alpha}_{i}$

$$
w_{\boldsymbol{\alpha}_{i}} \equiv \exp \left[-\frac{\pi}{2}\left(E_{\boldsymbol{\alpha}_{i}}-E_{-\boldsymbol{\alpha}_{i}}\right)\right] \in G .
$$

We can check that the $\Lambda$ 's given by Eq. (4.32) are fixed points by using the fact that the mass matrix transforms a: 10

$$
M \rightarrow w_{\boldsymbol{\alpha}_{i}} M w_{\boldsymbol{\alpha}_{i}}^{\dagger}=\left[\mathbf{m}-\left(\mathbf{m} \cdot \tilde{\boldsymbol{\alpha}}_{i}\right) \boldsymbol{\alpha}_{i}\right] \cdot \mathbf{H}, \quad \tilde{\boldsymbol{\alpha}}_{i}=2 \frac{\boldsymbol{\alpha}_{i}}{\boldsymbol{\alpha}_{i} \cdot \boldsymbol{\alpha}_{i}} .
$$

For example, $\Lambda_{h} w_{\boldsymbol{\alpha}_{i}}$ transforms under $U(1)_{M}$ as

$$
\Lambda_{h} w_{\boldsymbol{\alpha}_{i}} e^{i M \vartheta}=\Lambda_{h} e^{i w_{\boldsymbol{\alpha}_{i}}(M) \vartheta} w_{\boldsymbol{\alpha}_{i}}=e^{-i w_{\boldsymbol{\alpha}_{i}}\left(M_{n}\right) \vartheta} \Lambda_{h} w_{\boldsymbol{\alpha}_{i}} \sim \Lambda_{h} w_{\boldsymbol{\alpha}_{i}},
$$

where $w_{\boldsymbol{\alpha}_{i}}(M)$ and $w_{\boldsymbol{\alpha}_{i}}\left(M_{n}\right)$ are $2 n$-by- $2 n$ and $n$-by- $n$ matrices given by

$$
w_{\boldsymbol{\alpha}_{i}}(M)=\left(\begin{array}{l|l}
w_{\boldsymbol{\alpha}_{i}}\left(M_{n}\right) & \\
\hline & -w_{\boldsymbol{\alpha}_{i}}\left(M_{n}\right)
\end{array}\right)=\left[\mathbf{m}-\left(\mathbf{m} \cdot \tilde{\boldsymbol{\alpha}}_{i}\right) \boldsymbol{\alpha}_{i}\right] \cdot \mathbf{H} .
$$

Let us now calculate the magnetic flux $\sigma$ defined in Eq. (4.15) at each vacuum point. In terms of $\Lambda$, the flux $\sigma$ can be rewritten as

$$
\sigma=\operatorname{Tr}\left[\left(\Lambda \Lambda^{\dagger}\right)^{-1} \Lambda M \Lambda^{\dagger}\right]
$$

\footnotetext{
${ }^{10}$ Note that $w_{\boldsymbol{\alpha}_{i}}$ acts on any element of the Cartan subalgebra as the Weyl reflection of the coefficient vector $w_{\boldsymbol{\alpha}_{i}}(\mathbf{v} \cdot \mathbf{H}) w_{\boldsymbol{\alpha}_{i}}^{\dagger}=\left[\mathbf{v}-\left(\mathbf{v} \cdot \tilde{\boldsymbol{\alpha}}_{i}\right) \boldsymbol{\alpha}_{i}\right] \cdot \mathbf{H}$.
} 
For the highest weight vacuum $\Lambda_{h}$, the flux $\sigma$ is given by

$$
\sigma=-\operatorname{Tr} M_{n}=-2 \mathbf{m} \cdot \tilde{\boldsymbol{\nu}}_{h}
$$

Similarly, the flux $\sigma$ in the vacua $\Lambda_{h} w_{\boldsymbol{\alpha}_{i}}$ can be obtained by using Eq. (4.34) as

$$
\sigma=\operatorname{Tr}\left[\left(\Lambda_{h} \Lambda_{h}^{\dagger}\right)^{-1} \Lambda_{h} w_{\boldsymbol{\alpha}_{i}}(M) \Lambda_{h}^{\dagger}\right]=-2 \mathbf{m} \cdot w_{\boldsymbol{\alpha}_{i}}\left(\tilde{\boldsymbol{\nu}}_{h}\right)
$$

where $w_{\boldsymbol{\alpha}_{i}}\left(\tilde{\boldsymbol{\nu}}_{h}\right)$ is the coweight vector obtained from $\tilde{\boldsymbol{\nu}}_{h}$ by the Weyl reflection with respect to the simple root $\boldsymbol{\alpha}_{i}$

$$
w_{\boldsymbol{\alpha}_{i}}\left(\tilde{\boldsymbol{\nu}}_{h}\right) \equiv \tilde{\boldsymbol{\nu}}_{h}-\left(\tilde{\boldsymbol{\nu}}_{h} \cdot \boldsymbol{\alpha}_{i}\right) \tilde{\boldsymbol{\alpha}}_{i}
$$

As this example shows, the values of the magnetic flux at the vacuum points are specified by the coweights related to $\tilde{\boldsymbol{\nu}}_{h}$ via Weyl reflections. In the case of a single vortex in $G=$ $S O(2 n), U S p(2 n)$ theory, the orbit of $\tilde{\boldsymbol{\nu}}_{h}$ under Weyl reflections coincides with the whole set of weight vectors of the (Weyl) spinor representation of $\tilde{G}=S O(2 n), S O(2 n+1)$. Therefore the number of the vacua is the dimension of the representation 11

$$
n_{\text {vacua }}=\left\{\begin{array}{lll}
2^{n-1} & \text { (Weyl spinor rep. of } \tilde{G}=S O(2 n)) & \text { for } G=S O(2 n) \\
2^{n} & \text { (spinor rep. of } \tilde{G}=S O(2 n+1)) & \text { for } G=U S p(2 n)
\end{array} .\right.
$$

\section{Example 1: vacua of the $U S p(4) / U(2)$ sigma model}

Let us take the $U S p(4) / U(2)$ case as an example 12 The Weyl group is generated by the following elements corresponding to the simple roots $\boldsymbol{\alpha}_{1}=\boldsymbol{e}_{1}-\boldsymbol{e}_{2}$ and $\boldsymbol{\alpha}_{2}=2 \boldsymbol{e}_{2}$

$$
w_{\boldsymbol{\alpha}_{1}}=\left(\begin{array}{cc|cc}
0 & -1 & 0 & 0 \\
1 & 0 & 0 & 0 \\
\hline 0 & 0 & 0 & -1 \\
0 & 0 & 1 & 0
\end{array}\right), \quad w_{\boldsymbol{\alpha}_{2}}=\left(\begin{array}{cc|cc}
1 & 0 & 0 & 0 \\
0 & 0 & 0 & -1 \\
\hline 0 & 0 & 1 & 0 \\
0 & 1 & 0 & 0
\end{array}\right) .
$$

By using these group elements and $G L(n, \mathbb{C})$ transformations, we obtain the following vacuum matrices

$$
\begin{aligned}
\Lambda_{++}=\left(\begin{array}{ll|ll}
0 & 0 & 1 & 0 \\
0 & 0 & 0 & 1
\end{array}\right), & \Lambda_{+-}=\left(\begin{array}{ll|ll}
0 & 0 & 1 & 0 \\
0 & 1 & 0 & 0
\end{array}\right), \\
\Lambda_{-+}=\left(\begin{array}{ll|ll}
1 & 0 & 0 & 0 \\
0 & 0 & 0 & 1
\end{array}\right), & \Lambda_{--}=\left(\begin{array}{ll|ll}
1 & 0 & 0 & 0 \\
0 & 1 & 0 & 0
\end{array}\right),
\end{aligned}
$$

${ }^{11}$ We can also see that the number of vacua is the Euler characteristics of the target manifold [9]

$$
\chi\left(\frac{S O(2 n)}{U(n)}\right)=2^{n-1}, \quad \chi\left(\frac{U S p(2 n)}{U(n)}\right)=2^{n} .
$$

12 Note that $S O(6) / U(3) \cong U S p(4) / U(2) \cong \mathbb{C} P^{3}$. 
where we have used the Weyl group elements in the following way

$$
\Lambda_{++}=\Lambda_{h} \quad \stackrel{\alpha_{2}}{\rightarrow} \quad \Lambda_{+-} \sim \Lambda_{++} w_{\boldsymbol{\alpha}_{2}} \quad \stackrel{\alpha_{7}}{\rightarrow} \quad \Lambda_{-+} \sim \Lambda_{+-} w_{\boldsymbol{\alpha}_{1}} \stackrel{\boldsymbol{\alpha}_{2}}{\rightarrow} \quad \Lambda_{--} \sim \Lambda_{-+} w_{\boldsymbol{\alpha}_{2}} .
$$

The symbol $\sim$ denotes that we have used the equivalence relation (4.23). For each vacuum point on the target space, the coweight $\tilde{\boldsymbol{\nu}}$ and the magnetic flux, respectively, are given by

$$
\tilde{\boldsymbol{\nu}}_{ \pm \pm}=\frac{ \pm \boldsymbol{e}_{1} \pm \boldsymbol{e}_{2}}{2}, \quad \sigma_{ \pm \pm}=-\left( \pm m_{1} \pm m_{2}\right)
$$

Note that the signatures \pm correspond to the spins of the $S O(5)$ spinor representation. In general, the vacuum matrices for $G=U S p(2 n)$ has the following form

$$
\Lambda_{ \pm \cdots \pm}=\left(\begin{array}{ccc|ccc}
a_{1-} & & & a_{1+} & & \\
& \ddots & & & \ddots & \\
& & a_{n-} & & & a_{n+}
\end{array}\right),
$$

where $a_{i+}=1\left(a_{i+}=0\right)$ and $a_{i-}=0\left(a_{i-}=1\right)$ if the $i$-th signature of $\Lambda_{ \pm \ldots \pm}$ is $+(-)$.

\section{Example 2: vacua of the $S O(6) / U(3)$ sigma model}

In the case of $G=S O(6)$, the Weyl group is generated by the following elements corresponding to the simple roots $\boldsymbol{\alpha}_{1}=\boldsymbol{e}_{1}-\boldsymbol{e}_{2}, \boldsymbol{\alpha}_{2}=\boldsymbol{e}_{2}-\boldsymbol{e}_{3}$ and $\boldsymbol{\alpha}_{3}=\boldsymbol{e}_{2}+\boldsymbol{e}_{3}$

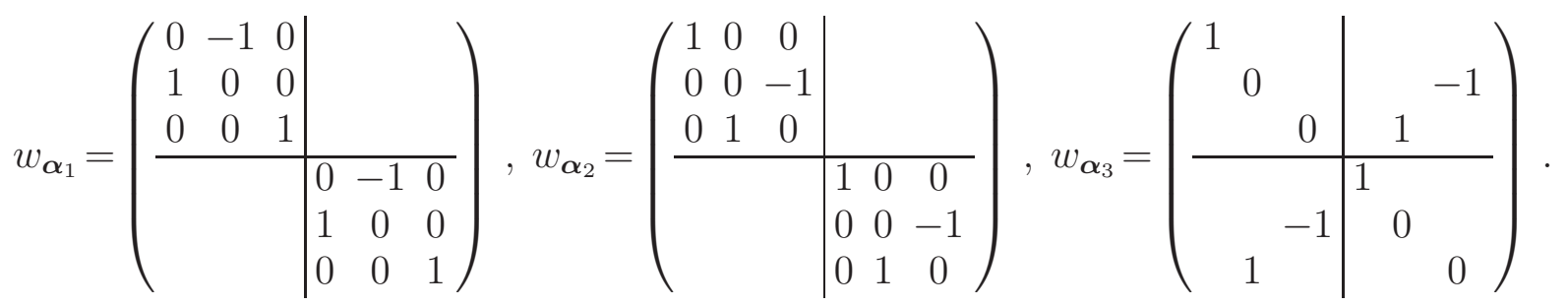

Combining these group elements with $G L(n, \mathbb{C})$ transformations (i.e. the equivalence relation), we obtain the following vacuum matrices

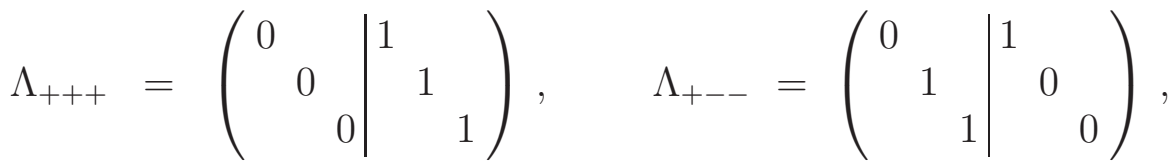

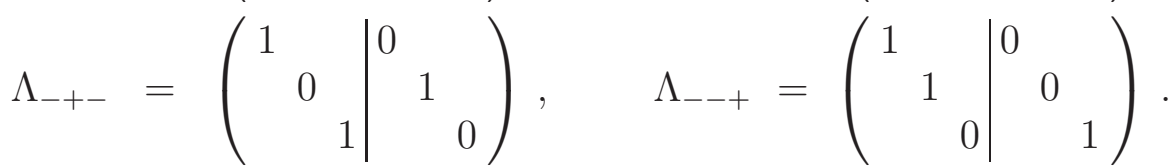

Here we have used the Weyl group elements in the following way

$$
\Lambda_{+++}=\Lambda_{h} \quad \stackrel{\alpha_{3}}{\rightarrow} \Lambda_{+--} \sim \Lambda_{+++} w_{\boldsymbol{\alpha}_{3}} \stackrel{\boldsymbol{\alpha}_{1}}{\rightarrow} \Lambda_{-+-} \sim \Lambda_{+--} w_{\boldsymbol{\alpha}_{1}} \stackrel{\boldsymbol{\alpha}_{2}}{\rightarrow} \Lambda_{--+} \sim \Lambda_{-+-} w_{\boldsymbol{\alpha}_{2}} .
$$

In these vacua, the coweight $\tilde{\boldsymbol{\nu}}$ and the magnetic flux are given by

$$
\tilde{\boldsymbol{\nu}}_{ \pm \pm \pm}=\frac{ \pm \boldsymbol{e}_{1} \pm \boldsymbol{e}_{2} \pm \boldsymbol{e}_{3}}{2}, \quad \sigma_{ \pm \pm}=-\left( \pm m_{1} \pm m_{2} \pm m_{3}\right)
$$


where the signatures \pm are the spins of the $S O(6)$ Weyl spinor representation. In general, the vacuum matrices for $G=S O(2 n)$ have the following form

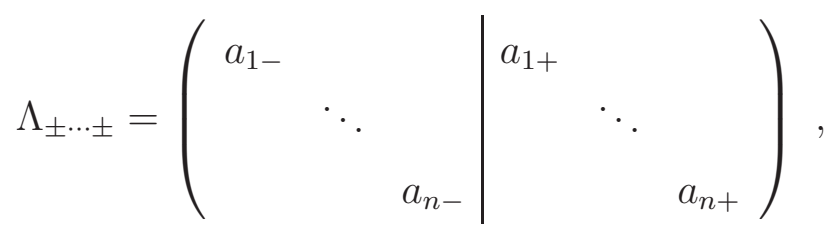

where the number of " - " $\mathrm{s}$ in $\Lambda_{ \pm \ldots \pm}$ is even while $a_{i+}=1\left(a_{i+}=0\right)$ and $a_{i-}=0\left(a_{i-}=1\right)$ if the $i$-th signature is $+(-)$.

\subsubsection{Single monopole configurations}

We have seen that the total mass of the BPS kinks is determined by the difference of the magnetic flux $\sigma$ at $x_{3} \rightarrow \pm \infty$, which is specified by the coweights, i.e. the weight vectors of the dual group $\tilde{G}$. It follows that the total mass of the kinks interpolating between two vacua labeled by $\tilde{\boldsymbol{\nu}}_{ \pm}$is given by

$$
M_{\mathrm{kink}}=\frac{4 \pi}{g^{2}} \mathbf{m} \cdot \mathrm{g}=-\frac{8 \pi}{g^{2}} \mathbf{m} \cdot\left(\tilde{\boldsymbol{\nu}}_{+}-\tilde{\boldsymbol{\nu}}_{-}\right)
$$

This shows that the magnetic charge vector $\mathbf{g}$ is proportional to the difference of the vectors $\tilde{\boldsymbol{\nu}}_{-}-\tilde{\boldsymbol{\nu}}_{+}$, which is an element of the coroot lattice, i.e. the lattice generated by the root vector of the dual group $\tilde{G}$. Since any element of the coroot lattice is a linear combination of the simple

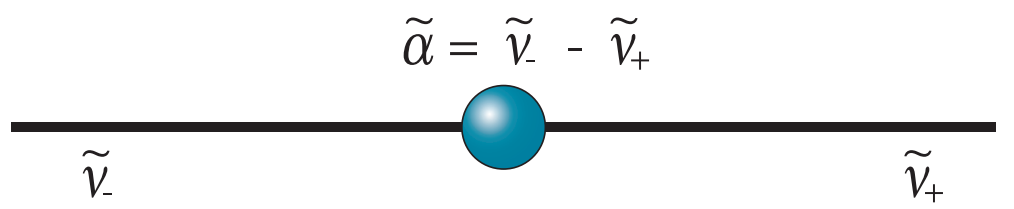

Fig. 2: A schematic picture of the composite of the vortices labeled by the coweight vectors $\tilde{\boldsymbol{\nu}}_{+}$ and $\tilde{\boldsymbol{\nu}}_{-}$and the monopole with the coroot $\tilde{\boldsymbol{\alpha}}=\tilde{\boldsymbol{\nu}}_{-}-\tilde{\boldsymbol{\nu}}_{+}$.

roots of $\tilde{G}$ with integer coefficients, the total mass can be decomposed into the masses of "the elementary kinks", which are given by the simple roots of the dual group

$$
M_{i}=\frac{8 \pi}{g^{2}} \mathbf{m} \cdot \tilde{\boldsymbol{\alpha}}_{i}, \quad(i=1,2, \ldots, \tilde{r}=\operatorname{rank} \tilde{G}) .
$$

These BPS masses of the elementary kinks coincide with those of the elementary monopoles appearing in the $4 d$ gauge theory with maximal gauge symmetry breaking

$$
S O(2 n) \rightarrow U(1)^{n}, \quad U S p(2 n) \rightarrow U(1)^{n}
$$


A schematic picture is shown in Fig. 2 .

As we can see from the BPS solution (4.22), the kink configuration is a one parameter flow on the target space parametrized by $x_{3}$. Similarly, this BPS flow can be rewritten in terms of $\Lambda$ as

$$
\Lambda\left(x_{3}\right)=\Lambda_{0} e^{M x_{3}}
$$

where $\Lambda_{0}$ is a constant $n$-by- $2 n$ matrix which specifies the kink configuration 13 As well as $\Lambda$, the matrix $\Lambda_{0}$ should obey the constraint and equivalence relation

$$
\Lambda_{0} J \Lambda_{0}^{\mathrm{T}}=0, \quad \Lambda_{0} \sim V \Lambda_{0}, \quad V \in G L(n, \mathbb{C}) .
$$

For example, the matrix $\Lambda_{0}$ for the BPS solution (4.22) is given by

$$
\Lambda_{0}=\left(B_{0} \mid \mathbf{1}_{n}\right) \text {. }
$$

By using the matrix $\Lambda_{0}$, the magnetic flux $\sigma$ for the kink configurations can be expressed as

$$
\sigma=\operatorname{Tr}\left[\left(\Lambda \Lambda^{\dagger}\right)^{-1} \Lambda M \Lambda^{\dagger}\right]=\frac{1}{2} \partial_{x_{3}} \log \operatorname{det}\left(\Lambda_{0} e^{2 M x_{3}} \Lambda_{0}^{\dagger}\right)
$$

In the following, we will discuss the kink configurations for a given matrix $\Lambda_{0}$. First let us consider the case with $\Lambda_{0}=\Lambda_{h}$. Since $e^{M x_{3}}$ acts trivially on $\Lambda_{h}$, the configuration is independent of $x_{3}$

$$
\Lambda\left(x_{3}\right)=\Lambda_{h} e^{M x_{3}} \sim \Lambda_{h}
$$

Therefore $\Lambda_{0}=\Lambda_{h}$ corresponds to the highest weight vortex vacuum. In general, the fixed points of $U(1)_{M}$ (generated by the Killing vectors $k^{i}$ ) correspond to those of the BPS flow (generated by $\left.i k^{i}\right)$

$$
\Lambda_{0} e^{i M \vartheta} \sim \Lambda_{0} \Longleftrightarrow \Lambda_{0} e^{M x_{3}} \sim \Lambda_{0}
$$

This means that if $\Lambda_{0}$ is one of the vacuum matrices, there is no kink in the configuration. The first example of the matrix $\Lambda_{0}$ which represents single kink configurations can be obtained by multiplying the highest weight vortex vacuum by the lowering operators $E_{-\boldsymbol{\alpha}}$ as

$$
\Lambda_{0}=\Lambda_{h} \exp \left[b E_{-\alpha}\right]
$$

where $b$ is a complex parameter. There is a caveat though, i.e. the action of the lowering operator $E_{-\boldsymbol{\alpha}}$ is non-trivial if and only if the inner product of $\tilde{\boldsymbol{\nu}}_{h}$ and $\boldsymbol{\alpha}$ is positive

$$
\tilde{\boldsymbol{\nu}}_{h} \cdot \boldsymbol{\alpha}>0
$$

13 This $n$-by-2n matrix $\Lambda_{0}$ is called the moduli matrix for domain walls [17, 18. 
In that case, the matrix $\Lambda\left(x_{3}\right)$ is given by

$$
\Lambda\left(x_{3}\right)=\Lambda_{h} \exp \left[b E_{-\boldsymbol{\alpha}}\right] e^{M x_{3}} \sim \Lambda_{h} \exp \left[b e^{(\boldsymbol{\alpha} \cdot \mathbf{m}) x_{3}} E_{-\boldsymbol{\alpha}}\right]
$$

where we have used that

$$
e^{-M x_{3}} E_{-\boldsymbol{\alpha}} e^{M x_{3}}=e^{(\boldsymbol{\alpha} \cdot \mathbf{m}) x_{3}} E_{-\boldsymbol{\alpha}} .
$$

Eq. (4.64) shows that the vortex configuration at $x_{3} \rightarrow-\infty$ is in the highest weight vacuum $\Lambda_{h}$. Note that we have fixed the ordering of the masses so that $\mathbf{m} \cdot \boldsymbol{\alpha}>0$ for all the positive roots $\boldsymbol{\alpha}$. To see the vortex configuration at $x_{3} \rightarrow \infty$, let us use the following decomposition of the group element

$$
\exp \left[a E_{-\boldsymbol{\alpha}}\right]=\exp \left[a^{-1} E_{\boldsymbol{\alpha}}\right] \exp [-\log a \tilde{\boldsymbol{\alpha}} \cdot \mathbf{H}] w_{\boldsymbol{\alpha}} \exp \left[a^{-1} E_{\boldsymbol{\alpha}}\right]
$$

Since the raising operator $E_{\boldsymbol{\alpha}}$ and the generator of the Cartan subalgebra $\mathbf{H}$ act on $\Lambda_{h}$ trivially, the matrix $\Lambda\left(x_{3}\right)$ can be rewritten as

$$
\Lambda\left(x_{3}\right) \sim\left(\Lambda_{h} w_{\boldsymbol{\alpha}}\right) \exp \left[b^{-1} e^{-(\boldsymbol{\alpha} \cdot \mathbf{m}) x_{3}} E_{\boldsymbol{\alpha}}\right] .
$$

Therefore the matrix $\Lambda\left(x_{3}\right)$ represents the kink between the vacua specified by $\tilde{\boldsymbol{\nu}}_{h}$ and $w_{\boldsymbol{\alpha}}\left(\tilde{\boldsymbol{\nu}}_{h}\right)$

$$
\lim _{x_{3} \rightarrow-\infty} \Lambda\left(x_{3}\right) \sim \Lambda_{h}, \quad \lim _{x_{3} \rightarrow \infty} \Lambda\left(x_{3}\right) \sim \Lambda_{h} w_{\boldsymbol{\alpha}} .
$$

Note that if $\tilde{\boldsymbol{\nu}}_{h} \cdot \boldsymbol{\alpha} \leq 0$, there does not exist any kink in the configuration since $\Lambda_{h} w_{\boldsymbol{\alpha}} \sim \Lambda_{h}$. As we have seen in Eq. (4.53), the mass of the kink is given by the difference of the coweights $\tilde{\boldsymbol{\nu}}_{ \pm}$ specifying the vacua at $x_{3} \rightarrow \pm \infty$. For $x_{3} \rightarrow-\infty$, the vacuum is specified by the highest weight vector $\tilde{\boldsymbol{\nu}}_{+}=\tilde{\boldsymbol{\nu}}_{h}$, while $\tilde{\boldsymbol{\nu}}_{-}$is given by Weyl reflection of $\tilde{\boldsymbol{\nu}}_{h}$ with respect to $\boldsymbol{\alpha}$. Since $\tilde{\boldsymbol{\nu}}_{h} \cdot \boldsymbol{\alpha} \leq 1$ for any root $\boldsymbol{\alpha}$, the Weyl reflection $w_{\boldsymbol{\alpha}}\left(\tilde{\boldsymbol{\nu}}_{h}\right)$ with respect to $\boldsymbol{\alpha}$ satisfying $\tilde{\boldsymbol{\nu}}_{h} \cdot \boldsymbol{\alpha}>0$ is given by

$$
w_{\boldsymbol{\alpha}}\left(\tilde{\boldsymbol{\nu}}_{h}\right)=\tilde{\boldsymbol{\nu}}_{h}-\left(\tilde{\boldsymbol{\nu}}_{h} \cdot \boldsymbol{\alpha}\right) \tilde{\boldsymbol{\alpha}}=\tilde{\boldsymbol{\nu}}_{h}-\tilde{\boldsymbol{\alpha}}
$$

Therefore, $\Lambda\left(x_{3}\right)$ represents the set of configurations of kinks interpolating between the vacua $\tilde{\boldsymbol{\nu}}_{h}$ and $\tilde{\boldsymbol{\nu}}_{h}-\tilde{\boldsymbol{\alpha}}$, whose mass is given by

$$
M_{\mathrm{kink}}=\frac{8 \pi}{g^{2}} \tilde{\boldsymbol{\alpha}} \cdot \mathbf{m} .
$$

The physical meaning of the parameter $b$ can be read off the profiles of the magnetic flux $\sigma$ and the energy density $\mathcal{E}=\frac{4 \pi}{g^{2}} \partial_{x_{3}} \sigma$

$$
\begin{aligned}
\sigma & =\tilde{\boldsymbol{\alpha}} \cdot \mathbf{m}\left[1+\tanh \left[\boldsymbol{\alpha} \cdot \mathbf{m}\left(x_{3}-r_{0}\right)\right]\right]-2 \tilde{\boldsymbol{\nu}}_{h} \cdot \mathbf{m}, \\
\mathcal{E} & =\frac{4 \pi}{g^{2}}(\tilde{\boldsymbol{\alpha}} \cdot \mathbf{m})(\boldsymbol{\alpha} \cdot \mathbf{m}) \operatorname{sech}^{2}\left[\boldsymbol{\alpha} \cdot \mathbf{m}\left(x_{3}-r_{0}\right)\right],
\end{aligned}
$$


where we have redefined the parameter $b$ as (See Eq.(4.62) $)$

$$
b=\exp \left[-(\boldsymbol{\alpha} \cdot \mathbf{m}) r_{0}+i \eta\right], \quad r_{0}, \eta \in \mathbb{R} .
$$

Since the kink profile of $\sigma$ and the energy density are functions of $x_{3}-r_{0}$ and independent of $\eta$, the parameters $r_{0}$ and $\eta$ can be interpreted as the position and internal phase moduli of the kink. The phase can be interpreted as the Nambu-Goldstone zero mode of the $U(1)_{M}$ symmetry broken by the kink. Note that the phase $\eta$ transforms under the $U(1)^{n}$ symmetry as

$$
\exp [i \boldsymbol{\theta} \cdot \mathbf{H}]: \eta \rightarrow \eta+\boldsymbol{\theta} \cdot \boldsymbol{\alpha} .
$$

As already mentioned, the kinks specified by the simple roots correspond to the elementary monopoles in the $4 d$ theory. There exists only one simple root 14 which satisfies the non-triviality condition $\tilde{\boldsymbol{\nu}}_{h} \cdot \boldsymbol{\alpha}>0$. Therefore only $\boldsymbol{\alpha}=\boldsymbol{\alpha}_{n}$ corresponds to the single elementary monopole for $\Lambda_{0}$ of the form (4.62). As we will see in the next section, the other cases are the coincident monopoles, which can be decomposed into several elementary monopoles.

Next, let us consider the action of the lowering operator on another vacuum

$$
\Lambda_{0}=\left(\Lambda_{h} w\right) \exp \left[b E_{-\boldsymbol{\alpha}}\right]=\Lambda_{h} \exp \left[b E_{-w(\boldsymbol{\alpha})}\right] w,
$$

where $w(\boldsymbol{\alpha})$ is the root vector obtained by the Weyl reflection. The action of $E_{-\boldsymbol{\alpha}}$ on $\Lambda_{h} w$ is non-trivial if and only if

$$
\tilde{\boldsymbol{\nu}}_{h} \cdot w(\boldsymbol{\alpha})=w\left(\tilde{\boldsymbol{\nu}}_{h}\right) \cdot \boldsymbol{\alpha}>0 .
$$

As in the previous case, we can show that

$$
\Lambda\left(x_{3}\right) \sim \Lambda_{h} w \exp \left[b e^{(\boldsymbol{\alpha} \cdot \mathbf{m}) x_{3}} E_{-\boldsymbol{\alpha}}\right] \sim \Lambda_{h} w w_{\boldsymbol{\alpha}} \exp \left[b^{-1} e^{-(\boldsymbol{\alpha} \cdot \mathbf{m}) x_{3}} E_{-\boldsymbol{\alpha}}\right] .
$$

Therefore the matrix $\Lambda\left(x_{3}\right)$ corresponds to the kink between the vacua specified by the vectors $w\left(\tilde{\boldsymbol{\nu}}_{h}\right)$ and $w\left(\tilde{\boldsymbol{\nu}}_{h}\right)-\tilde{\boldsymbol{\alpha}}$

$$
\lim _{x_{3} \rightarrow-\infty} \Lambda\left(x_{3}\right) \sim \Lambda_{h} w, \quad \lim _{x_{3} \rightarrow \infty} \Lambda\left(x_{3}\right) \sim \Lambda_{h} w w_{\boldsymbol{\alpha}} .
$$

The difference between this and the previous cases is that the vacua $\Lambda_{h}$ and $\Lambda_{h} w$ can admit different types of elementary monopoles (see Eqs. (4.63) and (4.76)). In general, the $i$-th elementary monopole can exist if and only if $i$-th Dynkin label $\tilde{l}_{i}$ of the coweight $\tilde{\boldsymbol{\nu}}$ at $x_{3} \rightarrow-\infty$ is positive

$$
\tilde{l}_{i} \equiv \tilde{\boldsymbol{\nu}} \cdot \boldsymbol{\alpha}_{i} \in \mathbb{Z}_{+} .
$$

Indeed, we can easily show that if the $i$-th Dynkin label is not positive, the action of the lowering operator $\exp \left[b E_{\boldsymbol{\alpha}_{i}}\right]$ is trivial

$$
\tilde{l}_{i} \leq 0 \Longleftrightarrow\left(\Lambda_{h} w\right) \exp \left[b E_{\boldsymbol{\alpha}_{i}}\right] \sim \Lambda_{h} w .
$$

${ }^{14} \boldsymbol{\alpha}_{n}=\boldsymbol{e}_{n-1}+\boldsymbol{e}_{n}$ and $\boldsymbol{\alpha}_{n}=2 \boldsymbol{e}_{n}$ for $G=S O(2 n)$ and $G=U S p(2 n)$, respectively. 
Example 1: elementary kinks in the $U S p(4) / U(2)$ sigma model

Let us first consider the elementary kinks in the $U S p(4) / U(2)$ sigma model. The Dynkin labels of the vacua $\tilde{l}_{i}=\boldsymbol{\alpha}_{i} \cdot \tilde{\boldsymbol{\nu}}$ are given by

$$
\begin{array}{lll}
\tilde{\boldsymbol{\nu}}_{++}=\frac{\boldsymbol{e}_{1}+\boldsymbol{e}_{2}}{2} & \leftrightarrow\left[\tilde{l}_{1}, \tilde{l}_{2}\right]=[0,1], & \tilde{\boldsymbol{\nu}}_{+-}=\frac{\boldsymbol{e}_{1}-\boldsymbol{e}_{2}}{2} \leftrightarrow\left[\tilde{l}_{1}, \tilde{l}_{2}\right]=[1,-1] \\
\tilde{\boldsymbol{\nu}}_{-+}=\frac{-\boldsymbol{e}_{1}+\boldsymbol{e}_{2}}{2} \leftrightarrow\left[\tilde{l}_{1}, \tilde{l}_{2}\right]=[-1,1], & \tilde{\boldsymbol{\nu}}_{--}=\frac{-\boldsymbol{e}_{1}-\boldsymbol{e}_{2}}{2} \leftrightarrow\left[\tilde{l}_{1}, \tilde{l}_{2}\right]=[0,-1]
\end{array}
$$

Therefore there is no elementary BPS kink between (--) and another vacuum 15 The lowering operators for the simple roots $\boldsymbol{\alpha}_{1}=\boldsymbol{e}_{1}-\boldsymbol{e}_{2}$ and $\boldsymbol{\alpha}_{2}=2 \boldsymbol{e}_{2}$ are given by

$$
\exp \left[b E_{-\boldsymbol{\alpha}_{1}}\right]=\left(\begin{array}{cc|cc}
1 & 0 & 0 & 0 \\
b & 1 & 0 & 0 \\
\hline 0 & 0 & 1 & -b \\
0 & 0 & 0 & 1
\end{array}\right), \quad \exp \left[b E_{-\boldsymbol{\alpha}_{2}}\right]=\left(\begin{array}{cc|cc}
1 & 0 & 0 & 0 \\
0 & 1 & 0 & 0 \\
\hline 0 & 0 & 1 & 0 \\
0 & b & 0 & 1
\end{array}\right)
$$

By using the vacuum matrices given in Eqs. (4.43) and (4.44), we find the following matrices $\Lambda_{0}$ for single elementary monopole configurations

$$
\begin{aligned}
& \Lambda_{(++,+-)}=\Lambda_{++} \exp \left[b E_{-\boldsymbol{\alpha}_{2}}\right]=\left(\begin{array}{ll|ll}
0 & 0 & 1 & 0 \\
0 & b & 0 & 1
\end{array}\right), \\
& \Lambda_{(+-,+)}=\Lambda_{+-} \exp \left[b E_{-\boldsymbol{\alpha}_{1}}\right]=\left(\begin{array}{ll|lc}
0 & 0 & 1 & -b \\
b & 1 & 0 & 0
\end{array}\right), \\
& \Lambda_{(-+,-)}=\Lambda_{-+} \exp \left[b E_{-\boldsymbol{\alpha}_{2}}\right]=\left(\begin{array}{ll|ll}
1 & 0 & 0 & 0 \\
0 & b & 0 & 1
\end{array}\right) .
\end{aligned}
$$

For these matrices, $\Lambda\left(x_{3}\right)=\Lambda_{0} e^{M x_{3}}$ correspond to the kinks interpolating between the vacua given in Eqs. (4.43) and (4.44). For example, $\Lambda_{(++,+-)}$represents the elementary kink between the $(++)$ and the $(+-)$ vacua

$$
\Lambda\left(x_{3}\right)=\Lambda_{(++,+-} e^{M x_{3}} \sim\left\{\begin{array}{l}
\left(\begin{array}{ll|ll}
0 & 0 & 1 & 0 \\
0 & a & 0 & 1
\end{array}\right) \underset{x_{3} \rightarrow-\infty}{\longrightarrow} \Lambda_{++} \\
\left(\begin{array}{ll|ll}
0 & 0 & 1 & 0 \\
0 & 1 & 0 & \frac{1}{a}
\end{array}\right) \underset{x_{3} \rightarrow \infty}{\longrightarrow} \Lambda_{+-}
\end{array}, \quad a \equiv b e^{2 m_{2} x_{3}},\right.
$$

where $M=\operatorname{diag}\left(m_{1}, m_{2},-m_{1},-m_{2}\right)$.

\footnotetext{
15 The kinks between (--) and the other vacua are anti-BPS monopoles.
} 
Example 2: elementary kinks in the $S O(6) / U(3)$ sigma model

In the case of the $S O(6) / U(3)$ sigma model, the Dynkin labels of the vacua $\tilde{l}_{i}=\boldsymbol{\alpha}_{i} \cdot \tilde{\boldsymbol{\nu}}$ are

$$
\begin{aligned}
& \tilde{\boldsymbol{\nu}}_{+++}=\frac{\boldsymbol{e}_{1}+\boldsymbol{e}_{2}+\boldsymbol{e}_{3}}{2} \quad \leftrightarrow \quad\left[\tilde{l}_{1}, \tilde{l}_{2}, \tilde{l}_{3}\right]=[0,0,1] \\
& \tilde{\boldsymbol{\nu}}_{+--}=\frac{\boldsymbol{e}_{1}-\boldsymbol{e}_{2}-\boldsymbol{e}_{3}}{2} \quad \leftrightarrow \quad\left[\tilde{l}_{1}, \tilde{l}_{2}, \tilde{l}_{3}\right]=[1,0,-1], \\
& \tilde{\boldsymbol{\nu}}_{-+-}=\frac{-\boldsymbol{e}_{1}+\boldsymbol{e}_{2}-\boldsymbol{e}_{3}}{2} \leftrightarrow\left[\tilde{l}_{1}, \tilde{l}_{2}, \tilde{l}_{3}\right]=[-1,1,0], \\
& \tilde{\boldsymbol{\nu}}_{--+}=\frac{-\boldsymbol{e}_{1}-\boldsymbol{e}_{2}-\boldsymbol{e}_{3}}{2} \leftrightarrow\left[\tilde{l}_{1}, \tilde{l}_{2}, \tilde{l}_{3}\right]=[0,0,-1] .
\end{aligned}
$$

The lowering operators for the simple roots $\boldsymbol{\alpha}_{1}=\boldsymbol{e}_{1}-\boldsymbol{e}_{2}, \boldsymbol{\alpha}_{2}=\boldsymbol{e}_{2}-\boldsymbol{e}_{3}$ and $\boldsymbol{\alpha}_{3}=\boldsymbol{e}_{2}+\boldsymbol{e}_{3}$ are given by

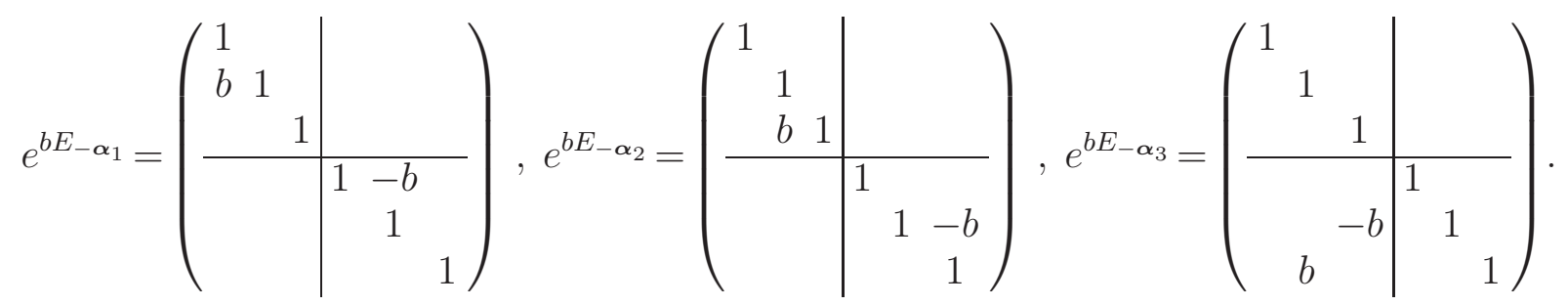

By using these group elements and the vacuum matrices given in Eqs. (4.48) and (4.49), we find the following $\Lambda_{0}$ corresponding to the elementary monopoles

$$
\begin{aligned}
& \Lambda_{(+++,+--)}=\Lambda_{+++} \exp \left[b E_{-\alpha_{3}}\right]=\left(\begin{array}{ccc|cc}
0 & & & & \\
& 0 & -b & & \\
& b & 0 & & \\
& & & 1
\end{array}\right)
\end{aligned}
$$

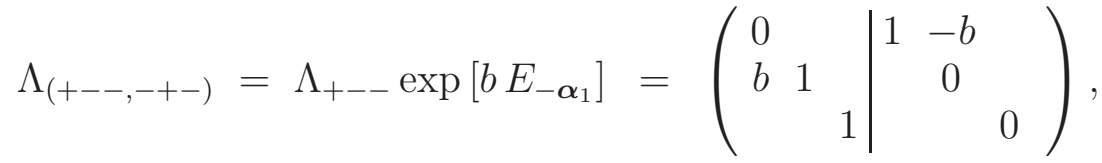

$$
\begin{aligned}
& \Lambda_{(-+-,-++)}=\Lambda_{-+-} \exp \left[b E_{-\alpha_{2}}\right]=\left(\begin{array}{cc|ccc}
1 & & 0 & & \\
& 0 & & 1 & -b \\
& b & 1 & & 0
\end{array}\right) .
\end{aligned}
$$

\subsubsection{Multi-monopole configurations}

As we have seen in the previous section, the lowering operators for the simple roots are "creation operators" of the elementary monopoles. Now let us consider the matrix $\Lambda_{0}$ with two lowering operators for different simple roots

$$
\Lambda_{0}=\left(\Lambda_{h} w\right) \exp \left[b_{i} E_{\boldsymbol{\alpha}_{i}}\right] \exp \left[b_{j} E_{\boldsymbol{\alpha}_{j}}\right], \quad i \neq j
$$


In this case, we have the following three expressions for the matrix $\Lambda\left(x_{3}\right)$

$$
\Lambda\left(x_{3}\right) \sim\left\{\begin{array}{ccc}
\left(\Lambda_{h} w\right) & \exp \left[b_{i} e^{\left(\boldsymbol{\alpha}_{i} \cdot \mathbf{m}\right) x_{3}} E_{-\boldsymbol{\alpha}_{i}}\right] & \exp \left[b_{j} e^{\left(\boldsymbol{\alpha}_{j} \cdot \mathbf{m}\right) x_{3}} E_{-\boldsymbol{\alpha}_{j}}\right] \\
\left(\Lambda_{h} w w_{\boldsymbol{\alpha}_{i}}\right) & \exp \left[b_{i}^{-1} e^{-\left(\boldsymbol{\alpha}_{i} \cdot \mathbf{m}\right) x_{3}} E_{\boldsymbol{\alpha}_{i}}\right] & \exp \left[b_{j} e^{\left(\boldsymbol{\alpha}_{j} \cdot \mathbf{m}\right) x_{3}} E_{-\boldsymbol{\alpha}_{j}}\right] \\
\left(\Lambda_{h} w w_{\boldsymbol{\alpha}_{i}} w_{\boldsymbol{\alpha}_{j}}\right) & \exp \left[b_{j}^{-1} e^{-\left(\boldsymbol{\alpha}_{j} \cdot \mathbf{m}\right) x_{3}} E_{\boldsymbol{\alpha}_{j}}\right] \exp \left[b_{i}^{-1} e^{-\left(\boldsymbol{\alpha}_{i} \cdot \mathbf{m}\right) x_{3}} E_{\boldsymbol{\alpha}_{i}}\right]
\end{array}\right.
$$

where we have used the fact that $\left[E_{\boldsymbol{\alpha}_{i}}, E_{-\boldsymbol{\alpha}_{j}}\right]=0$ for any simple roots $\boldsymbol{\alpha}_{i}$ and $\boldsymbol{\alpha}_{j}(i \neq j)$. If we set the moduli parameters a 16

$$
\log b_{i}=-\left(\boldsymbol{\alpha}_{i} \cdot \mathbf{m}\right) r_{i}+i \eta_{i}, \quad \log b_{j}=-\left(\boldsymbol{\alpha}_{j} \cdot \mathbf{m}\right) r_{j}+i \eta_{j},
$$

we find that the matrix $\Lambda\left(x_{3}\right)$ flows though the following three vacua

$$
\Lambda\left(x_{3}\right) \sim\left\{\begin{array}{cl}
\Lambda_{h} w & x_{3} \ll r_{i} \\
\Lambda_{h} w w_{\boldsymbol{\alpha}_{i}} & r_{i} \ll x_{3} \ll r_{j} \\
\Lambda_{h} w w_{\boldsymbol{\alpha}_{i}} w_{\boldsymbol{\alpha}_{j}} & r_{j} \ll x_{3}
\end{array}\right.
$$

where we have assumed $r_{i} \ll r_{j}$. Eq. (4.98) shows that $\Lambda\left(x_{3}\right)$ corresponds to the configuration of two elementary kinks $\tilde{\boldsymbol{\alpha}}_{i}$ and $\tilde{\boldsymbol{\alpha}}_{j}$ located at $x_{3}=r_{i}$ and $x_{3}=r_{j}$, respectively

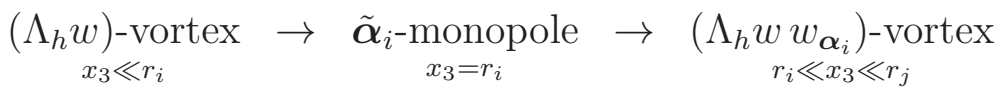

$$
\begin{aligned}
& \rightarrow \underset{x_{3}=r_{j}}{\tilde{\boldsymbol{\alpha}}_{j^{-}} \text {-monopole }} \rightarrow \underset{r_{j} \ll x_{3}}{\left(\Lambda_{h} w w_{\boldsymbol{\alpha}_{i}} w_{\boldsymbol{\alpha}_{j}}\right) \text {-vortex }} .
\end{aligned}
$$

If the lowering operators $E_{-\boldsymbol{\alpha}_{i}}$ and $E_{-\boldsymbol{\alpha}_{j}}$ commute with each other, we have another expression for the matrix $\Lambda\left(x_{3}\right)$

$$
\begin{aligned}
\Lambda\left(x_{3}\right) & \sim\left(\Lambda_{h} w w_{\boldsymbol{\alpha}_{j}}\right) \exp \left[b_{j}^{-1} e^{-\left(\boldsymbol{\alpha}_{j} \cdot \mathbf{m}\right) x_{3}} E_{\boldsymbol{\alpha}_{j}}\right] \exp \left[b_{i} e^{\left(\boldsymbol{\alpha}_{i} \cdot \mathbf{m}\right) x_{3}} E_{-\boldsymbol{\alpha}_{i}}\right] \\
& \approx \Lambda_{h} w w_{\boldsymbol{\alpha}_{j}}\left(r_{i} \gg x_{3} \gg r_{j}\right) .
\end{aligned}
$$

Therefore the ordering of the monopoles can be exchanged if the corresponding operators satisfy $\left[E_{-\boldsymbol{\alpha}_{i}}, E_{-\boldsymbol{\alpha}_{j}}\right]=0$

$$
\begin{aligned}
& \underset{x_{3} \ll r_{j}}{\left(\Lambda_{h} w\right) \text {-vortex }} \rightarrow \underset{x_{3}=r_{j}}{\tilde{\boldsymbol{\alpha}}_{j^{\prime}} \text {-monopole }} \rightarrow \underset{r_{j} \ll x_{3} \ll r_{i}}{\left(\Lambda_{h} w w_{\boldsymbol{\alpha}}\right) \text {-vortex }} \\
& \rightarrow \underset{x_{3}=r_{i}}{\tilde{\boldsymbol{\alpha}}_{i} \text {-monopole }} \rightarrow \underset{\left(\Lambda_{h} w w_{\boldsymbol{\alpha}_{i}} w_{\boldsymbol{\alpha}_{j}}\right) \text {-vortex }}{r_{i} \ll x_{3}} .
\end{aligned}
$$

On the other hand, if the lowering operators do not commute $\left[E_{-\boldsymbol{\alpha}_{i}}, E_{-\boldsymbol{\alpha}_{j}}\right] \neq 0$, the monopoles have a fixed ordering, which was found for the Grassmannian sigma model [18]. To see this, let us consider the following example in the $U S p(4) / U(2)$ sigma model

$$
\Lambda\left(x_{3}\right)=\Lambda_{++} \exp \left[b_{2} E_{-\boldsymbol{\alpha}_{2}}\right] \exp \left[b_{1} E_{-\boldsymbol{\alpha}_{1}}\right] e^{M x_{3}} \sim\left(\begin{array}{cc|cc}
a_{1}^{2} a_{2} & a_{1} a_{2} & 1 & 0 \\
a_{1} a_{2} & a_{2} & 0 & 1
\end{array}\right)
$$

${ }^{16}$ There is no summation on $i$ and $j$ in Eq. (4.97). 
where $a_{1}$ and $a_{2}$ are given by

$$
\begin{aligned}
& a_{1}=b_{1} e^{\left(m_{1}-m_{2}\right) x_{3}}=e^{\left(m_{1}-m_{2}\right)\left(x_{3}-r_{1}\right)+i \eta_{1}}, \\
& a_{2}=b_{2} e^{2 m_{2} x_{3}}=e^{2 m_{2}\left(x_{3}-r_{2}\right)+i \eta_{2}}
\end{aligned}
$$

In this configuration, there are two monopoles at $x_{3}=r_{2}$ and $x_{3}=r_{1}$ if $r_{2}<r_{1}$. Let us take the limits $r_{2} \rightarrow \infty$ and $r_{1} \rightarrow-\infty$ while keeping $a_{1}^{2} a_{2}$ fixed

$$
\Lambda\left(x_{3}\right) \sim\left(\begin{array}{cc|cc}
a_{1+2} & 0 & 1 & 0 \\
0 & 0 & 0 & 1
\end{array}\right) \sim \Lambda_{++} \exp \left[a_{1+2} E_{-\left(\boldsymbol{\alpha}_{1}+\boldsymbol{\alpha}_{2}\right)}\right]
$$

where $a_{1+2}$ takes the form $a_{1+2}=\exp \left[\left(m_{1}+m_{2}\right)\left(x_{3}-r_{1+2}\right)+i \eta_{1+2}\right]$. Eq. (4.104) corresponds to a single monopole with root vector $\tilde{\boldsymbol{\alpha}}_{1}+\tilde{\boldsymbol{\alpha}}_{2}$. Therefore the monopoles with $\tilde{\boldsymbol{\alpha}}_{1,2}$ cannot be exchanged and become a single monopole with $\tilde{\boldsymbol{\alpha}}_{1}+\tilde{\boldsymbol{\alpha}}_{2}$ in the coincident limit.

To understand the ordering of the monopoles, it is convenient to introduce the following matrix 17

$$
\Sigma\left(x_{3}\right) \equiv\left(\Lambda \Lambda^{\dagger}\right)^{-1} \Lambda M \Lambda^{\dagger}
$$

Since $\operatorname{Tr} \Sigma=\sigma$ is the magnetic flux which has a kink profile, the eigenvalues of $\Sigma$ also have kink profiles, which contain more information on the ordering of the kinks, as in the case of Grassmannian sigma model [43. Fig. 3 shows the coincident limit of the $\boldsymbol{\alpha}_{1^{-}}$and $\boldsymbol{\alpha}_{2}$-monopoles in the $U S p(4)$ case.

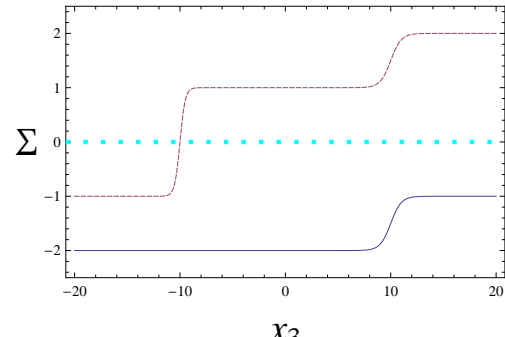

$r_{1}=10, r_{2}=-10$

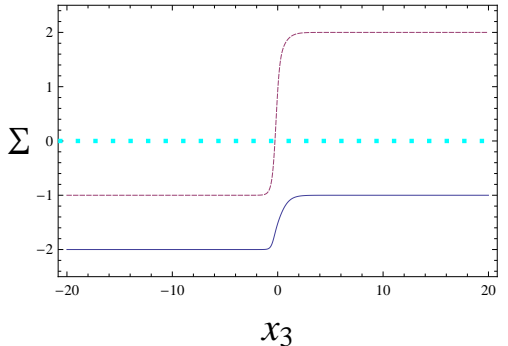

$r_{1}=0, r_{2}=0$

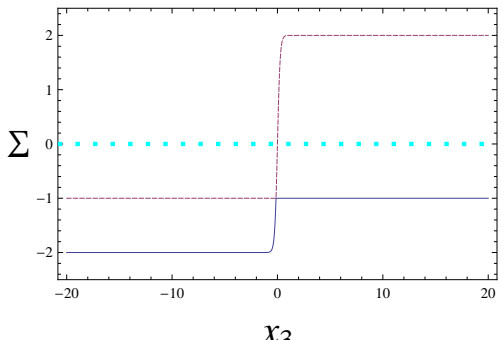

$r_{1}=-10, r_{2}=10$

Fig. 3: The eigenvalues of $\Sigma$ as functions of $x_{3}\left(G=U S p(4), m_{1}=2, m_{2}=1\right)$.

In general, the matrix $\Sigma$ has the following properties:

1. The eigenvalues of $\Sigma$ are increasing functions of $x_{3}$.

\footnotetext{
17 The definition of $\Sigma$ here is different from that is used in the Grassmannian sigma model [17, 18] by a matrix conjugation $\Sigma \rightarrow S^{-1} \Sigma S$.
} 
2. At the vacuum points, all of the eigenvalues are different and given by the mass parameters $\pm m_{i}$.

3. If $m_{i}\left(-m_{i}\right)$ is one of the eigenvalues, $-m_{i}\left(m_{i}\right)$ is not contained in the set of eigenvalues.

4. For the vacuum $\Lambda_{s_{1} \cdots s_{n}}\left(s_{i}= \pm\right)$, the eigenvalues are $-s_{1} m_{1}, \cdots,-s_{n} m_{n}$.

With these rules in mind, we can draw the generic kink profiles of eigenvalues diagrammatically in the thin wall limit 18 (see $U S p(6)$ and $S O(8)$ examples in Figs.4 and 5). In the $S U(N)$ case, the profiles of eigenvalues of $\Sigma$ were interpreted as kinky D-brane configurations 43 .

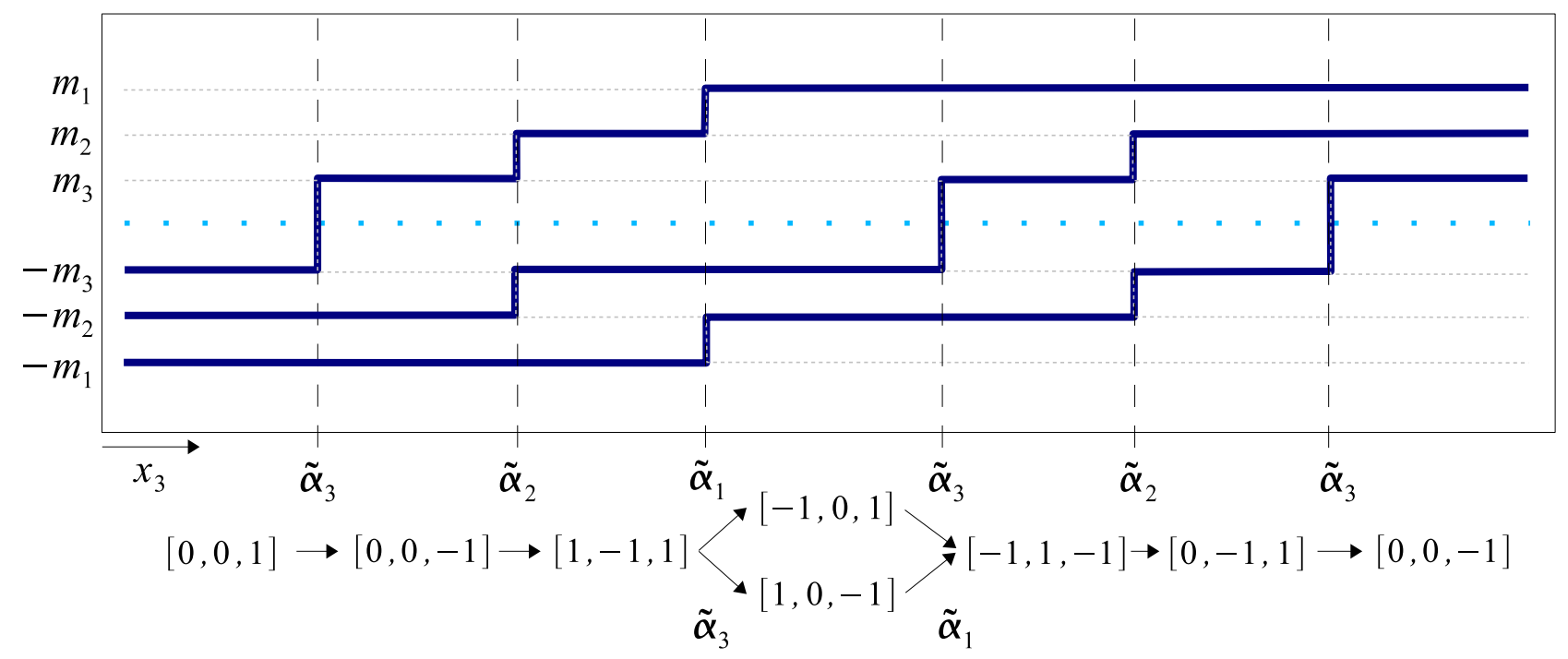

Fig. 4: The $U S p(6)$ maximal kink configuration in the thin wall limit. The eigenvalues of $\Sigma$ (solid lines) and their mirror image (dashed lines) with respect to the line $\Sigma=0$ (dotted line) are all different in each vortex vacuum (the regions are separated by vertical dashed lines). The sequence of Dynkin labels of the $S O(7)$ spinor is assigned so that the subsequent labels are obtained by subtracting the row of the Cartan matrix $\tilde{C}$ corresponding to the coroot of the monopole between the vacua. The branch of the sequence corresponds to commutative monopoles.

Finally, let us discuss the most generic configurations of kinks. The matrix $\Lambda_{0}$ for the generic configuration can be obtained from $\Lambda_{h}$ by multiplying as many lowering operators as possible

$$
\Lambda_{0}=\Lambda_{h} \exp \left[b_{i_{1}} E_{-\boldsymbol{\alpha}_{i_{1}}}\right] \cdots \exp \left[b_{i_{p}} E_{-\boldsymbol{\alpha}_{i_{p}}}\right]
$$

\footnotetext{
18 The thin wall limit can be interpreted as the large mass limit in which the kink profile becomes a step function $\lim _{m \rightarrow \infty}[1+\tanh (m x)]=2 \theta(x)$ where $\theta(x)$ stands for the step function. In the $S U(N)$ case a different limit was taken in Ref. [45] to obtain kinks with constant slopes, in order to study the statistical mechanics of non-Abelian vortices.
} 


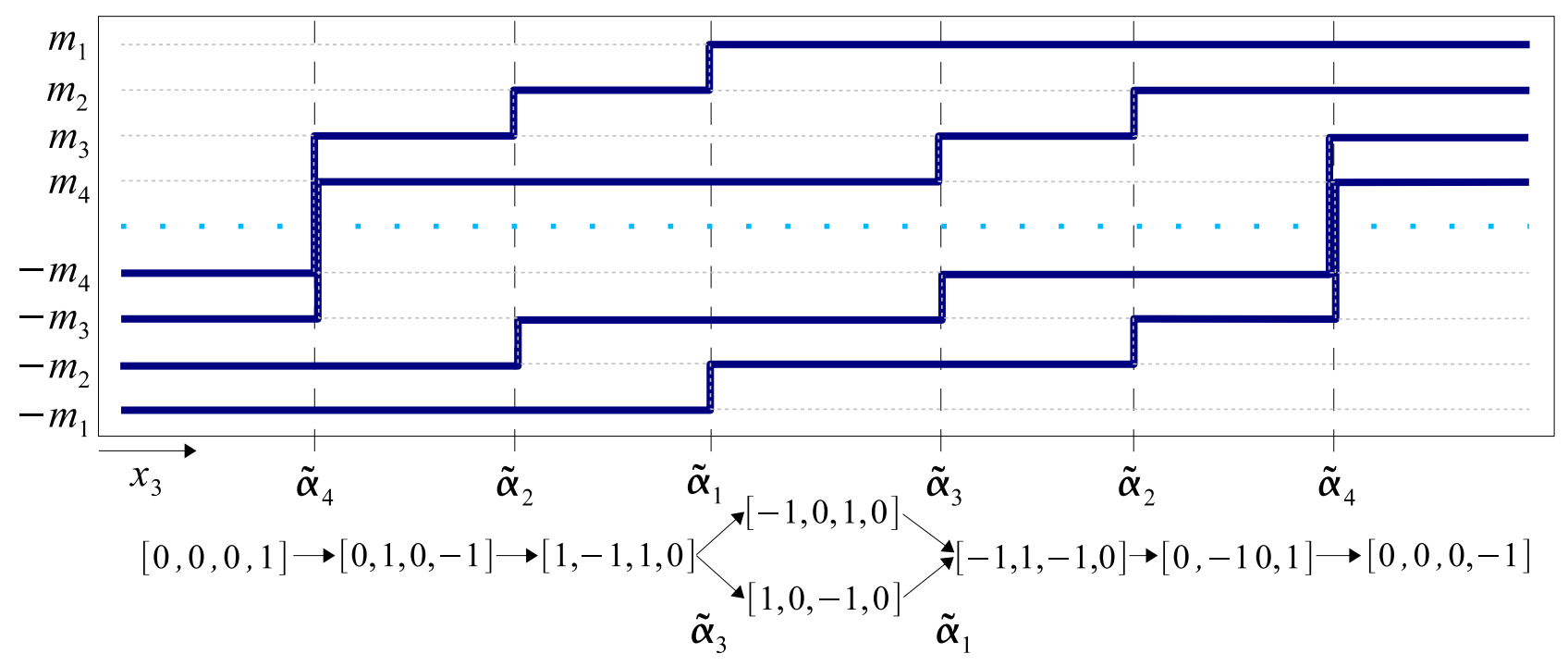

Fig. 5: The $S O(8)$ maximal kink configuration in the thin wall limit and the sequence of Dynkin labels of the $S O(8)$ Weyl spinor. Each eigenvalue (solid lines) can cross the dotted line $\Sigma=0$ only if it is paired with another eigenvalue. Because of the vacuum condition, two kinks must go up simultaneously across the dotted line.

where $\boldsymbol{\alpha}_{i_{j}}$ are simple roots. Since the multiplication of the lowering operators becomes trivial at a finite number $p$, there exist "maximal kink configurations" containing the maximal number of kinks. As shown in Appendix C, we can always rewrite the matrix $\Lambda_{0}$ as

$$
\begin{aligned}
\Lambda_{0} & \sim\left(\Lambda_{h} w_{\boldsymbol{\alpha}_{i_{1}}}\right) \exp \left[b_{i_{2}}^{\prime} E_{-\boldsymbol{\alpha}_{i_{2}}}\right] \cdots \exp \left[b_{i_{p}}^{\prime} E_{-\boldsymbol{\alpha}_{i_{p}}}\right] \exp \left[b_{i_{1}}^{\prime}{ }^{-1} E_{\boldsymbol{\alpha}_{i_{1}}}\right] \\
& \sim\left(\Lambda_{h} w_{\boldsymbol{\alpha}_{i_{1}}} w_{\boldsymbol{\alpha}_{i_{2}}}\right) \exp \left[b_{i_{3}}^{\prime \prime} E_{-\boldsymbol{\alpha}_{i_{3}}}\right] \cdots \exp \left[b_{i_{p}}^{\prime \prime} E_{-\boldsymbol{\alpha}_{i_{p}}}\right] \exp \left[b_{i_{2}}^{\prime \prime}{ }^{-1} E_{\boldsymbol{\alpha}_{i_{2}}}\right] \exp \left[b_{i_{1}}^{\prime \prime}{ }^{-1} E_{\boldsymbol{\alpha}_{i_{1}}}\right] \\
& \sim \cdots
\end{aligned}
$$

Repeating this procedure, we can read off the vortex-monopole configuration from $x_{3} \rightarrow-\infty$ to $x_{3} \rightarrow \infty$

$$
\Lambda_{h} \text {-vortex } \rightarrow \tilde{\boldsymbol{\alpha}}_{i_{1}} \text {-monopole } \rightarrow\left(\Lambda_{h} w_{\boldsymbol{\alpha}_{i}}\right) \text {-vortex } \rightarrow \cdots \rightarrow\left(\Lambda_{h} w_{\boldsymbol{\alpha}_{i_{1}}} \cdots w_{\boldsymbol{\alpha}_{i_{p}}}\right) \text {-vortex }
$$

The lowering operator $\exp \left[b_{i} E_{-\boldsymbol{\alpha}_{i}}\right]$ creates the $\tilde{\boldsymbol{\alpha}}_{i}$-monopole if the vortex has the coweight $\tilde{\boldsymbol{\nu}}$ with a positive Dynkin label $\tilde{l}_{i}=\tilde{\boldsymbol{\nu}} \cdot \boldsymbol{\alpha}_{i}$. Then it is connected to the vortex with the coweight $\tilde{\boldsymbol{\nu}}-\tilde{\boldsymbol{\alpha}}_{i}$ whose Dynkin labels are $\tilde{l}_{j}^{\prime}=\tilde{l}_{j}-\tilde{C}_{i j}$. Here $\tilde{C}_{i j}$ is an element of the Cartan matrix

$$
\tilde{C}_{i j} \equiv \tilde{\boldsymbol{\alpha}}_{i} \cdot \boldsymbol{\alpha}_{j}
$$

Therefore, the most generic $S O(2 n)$ and $U S p(2 n)$ vortex-monopole configurations can be constructed in the same way as the (Weyl) spinor representation of $S O(2 n)$ and $S O(2 n+1)$ (see the $U S p(6)$ and $S O(8)$ examples given in Figs.4 and 5 ). 


\section{Example 1: maximal kink configuration in the $U S p(6) / U(3)$ sigma model}

Let us consider the maximal kink configuration in the $U S p(6) / U(3)$ sigma model. The most generic form of the matrix $\Lambda_{0}$ is given by

$$
\begin{aligned}
\Lambda_{0}= & \Lambda_{h} \exp \left[b_{3,1} E_{-\boldsymbol{\alpha}_{3}}\right] \exp \left[b_{2,1} E_{-\boldsymbol{\alpha}_{2}}\right] \exp \left[b_{1,1} E_{-\boldsymbol{\alpha}_{1}}\right] \\
& \times \exp \left[b_{3,2} E_{-\boldsymbol{\alpha}_{3}}\right] \exp \left[b_{2,2} E_{-\boldsymbol{\alpha}_{2}}\right] \\
& \times \exp \left[b_{3,3} E_{-\boldsymbol{\alpha}_{3}}\right] .
\end{aligned}
$$

The number of moduli parameters $b_{i, j}$ is in accordance with the number of the matrix elements of the symmetric matrix $B_{0}$. The corresponding kink profile and the sequence of Dynkin labels of the $S O(7)$ spinor representation are given in Fig.4.

In general, the matrix $\Lambda_{0}$ for the maximal kink configurations in the $U S p(2 n) / U(n)$ sigma model is given by

$$
\begin{aligned}
\Lambda_{0}= & \Lambda_{h} \exp \left[b_{n, 1} E_{-\boldsymbol{\alpha}_{n}}\right] \cdots \exp \left[b_{2,1} E_{-\boldsymbol{\alpha}_{2}}\right] \exp \left[b_{1,1} E_{-\boldsymbol{\alpha}_{1}}\right] \\
& \times \exp \left[b_{n, 2} E_{-\boldsymbol{\alpha}_{n}}\right] \cdots \exp \left[b_{2,2} E_{-\boldsymbol{\alpha}_{2}}\right] \\
& \vdots \\
& \times \exp \left[b_{n, n} E_{-\boldsymbol{\alpha}_{n}}\right] .
\end{aligned}
$$

There are $i(i=1, \ldots, n) \tilde{\boldsymbol{\alpha}}_{i}$-monopoles in the maximal configuration and the complex parameters $b_{i, j}(j=1, \ldots, i)$ are the position and phase moduli of $j$-th $\tilde{\boldsymbol{\alpha}}_{i}$-monopole.

\section{Example 2: maximal kink configuration in the $S O(8) / U(4)$ sigma model}

The matrix $\Lambda_{0}$ for the maximal kink configuration in the $S O(8) / U(4)$ sigma model is given by

$$
\begin{aligned}
\Lambda_{0}= & \Lambda_{h} \exp \left[b_{4,1} E_{-\boldsymbol{\alpha}_{4}}\right] \exp \left[b_{2,1} E_{-\boldsymbol{\alpha}_{2}}\right] \exp \left[b_{1,1} E_{-\boldsymbol{\alpha}_{1}}\right] \\
& \times \exp \left[b_{3,2} E_{-\boldsymbol{\alpha}_{3}}\right] \exp \left[b_{2,2} E_{-\boldsymbol{\alpha}_{2}}\right] \\
& \times \exp \left[b_{4,3} E_{-\boldsymbol{\alpha}_{4}}\right] .
\end{aligned}
$$

As in the case of $U S p(6) / U(3)$, the number of moduli parameters $b_{i, j}$ is in accordance with that of the matrix elements of the anti-symmetric matrix $B_{0}$. The kink profiles and the sequence of Dynkin labels of the $S O(8)$ Weyl spinor representation are given in Fig.5.

The generic form of the matrix $\Lambda_{0}$ for the maximal kink configurations in the $S O(2 n) / U(n)$ 
sigma model is given by

$$
\begin{aligned}
\Lambda_{0}= & \Lambda_{h} \exp \left[b_{n, 1} E_{-\boldsymbol{\alpha}_{n}}\right] \exp \left[b_{n-2,1} E_{-\boldsymbol{\alpha}_{n-2}}\right] \cdots \exp \left[b_{2,1} E_{-\boldsymbol{\alpha}_{2}}\right] \exp \left[b_{1,1} E_{-\boldsymbol{\alpha}_{1}}\right] \\
& \times \exp \left[b_{n-1,2} E_{-\boldsymbol{\alpha}_{n-1}}\right] \cdots \exp \left[b_{2,2} E_{-\boldsymbol{\alpha}_{2}}\right] \\
& \times \exp \left[b_{n, 3} E_{-\boldsymbol{\alpha}_{n}}\right] \exp \left[b_{n-2,3} E_{-\boldsymbol{\alpha}_{n-2}}\right] \cdots \exp \left[b_{3,3} E_{-\boldsymbol{\alpha}_{3}}\right] \\
& \times \exp \left[b_{n-1,4} E_{-\boldsymbol{\alpha}_{n-1}}\right] \cdots \exp \left[b_{4,4} E_{-\boldsymbol{\alpha}_{4}}\right] \\
& \vdots \\
& \times L
\end{aligned}
$$

where the last operator $L$ is

$$
L=\left\{\begin{array}{cl}
\exp \left[b_{n, n} E_{-\boldsymbol{\alpha}_{n}}\right] & \text { for } S O\left(4 n^{\prime}+4\right) \\
\exp \left[b_{n-1, n} E_{-\boldsymbol{\alpha}_{n-1}}\right] & \text { for } S O\left(4 n^{\prime}+2\right)
\end{array} .\right.
$$

For $S O\left(4 n^{\prime}+2\right)\left(S O\left(4 n^{\prime}+4\right)\right)$, the numbers of $\tilde{\boldsymbol{\alpha}}_{i}$-monopoles in the maximal configuration are

$$
\# \tilde{\boldsymbol{\alpha}}_{i \text {-monopoles }}=\left\{\begin{array}{ll}
i & \text { for } i=1, \cdots, n-2 \\
n^{\prime} & \text { for } i=n-1 \\
n^{\prime}\left(n^{\prime}+1\right) & \text { for } i=n
\end{array} .\right.
$$

We can also discuss the kinks on the vortex with opposite $\mathbb{Z}_{2}$ topological charge (chirality) by starting from the following highest weight vacuum

$$
\Lambda_{h}^{\prime}=\left(\begin{array}{ll|lll}
\mathbf{0}_{n-1} & & \mathbf{1}_{n-1} & \\
& 1 & & 0
\end{array}\right) .
$$

In the case of $S O(4)$, the vortices with different $\mathbb{Z}_{2}$ topological charge admit different types of elementary monopoles (see Fig.6)

$$
\begin{aligned}
& \Lambda_{(++,-)}=\Lambda_{h} \exp \left[b E_{-\boldsymbol{\alpha}_{1}}\right]=\left(\begin{array}{cc|cc}
0 & -b & 1 & 0 \\
b & 0 & 0 & 1
\end{array}\right), \\
& \Lambda_{(+-,-+)}=\Lambda_{h}^{\prime} \exp \left[b E_{-\boldsymbol{\alpha}_{2}}\right]=\left(\begin{array}{cc|cc}
0 & 0 & 1 & -b \\
b & 1 & 0 & 0
\end{array}\right),
\end{aligned}
$$

where $\boldsymbol{\alpha}_{1}=\boldsymbol{e}_{1}-\boldsymbol{e}_{2}$ and $\boldsymbol{\alpha}_{2}=\boldsymbol{e}_{1}+\boldsymbol{e}_{2}$.

\section{Witten's effect on the vortex worldsheet}

In this section we consider the dyonic configuration. The bulk $\theta$-term induces a $\theta$-term on the vortex worldsheet, which can be obtained by substituting the solution (2.40) into the bulk $\theta$-term

$$
\mathcal{L}_{\theta}=\frac{\theta}{32 \pi^{2}} \epsilon^{\mu \nu \rho \sigma} \int d^{4} x \operatorname{Tr}\left(F_{\mu \nu} F_{\rho \sigma}\right)=\frac{\theta}{2 \pi} \int d^{2} x \frac{i g^{2}}{4 \pi} g_{i \bar{j}} \epsilon^{\alpha \beta} \partial_{\alpha} b^{i} \partial_{\beta} \bar{b}^{\bar{j}}
$$



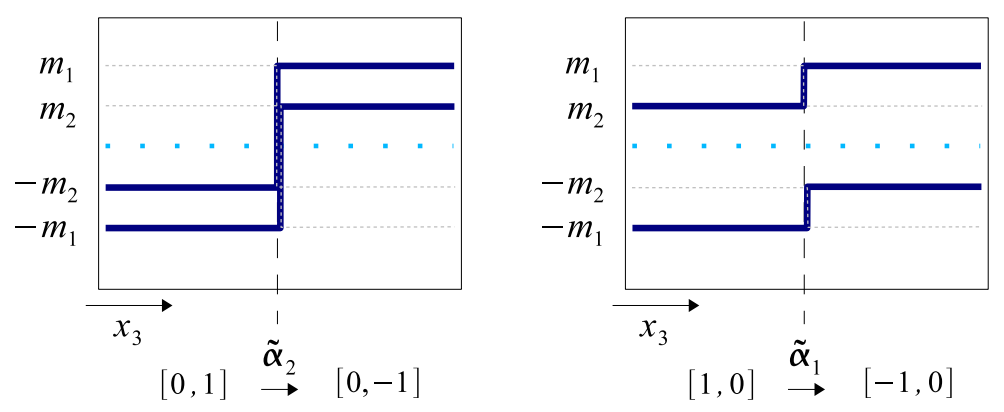

Fig. 6: The $S O(4)$ kink configurations on the vortices with opposite $\mathbb{Z}_{2}$ topological charge.

where $\epsilon^{t x_{3}}=-\epsilon^{x_{3} t}=1$. This induced $\theta$-term on the vortex worldsheet is proportional to the pullback of the Kähler form onto the vortex worldsheet. For the $S O(2 n) / U(n)$ and $U S p(2 n) / U(n)$ cases, the explicit form of the induced $\theta$-term is given by

$$
\mathcal{L}_{\theta}=-i \frac{\theta}{2 \pi} \epsilon^{\alpha \beta} \operatorname{Tr}\left[X^{-1} \partial_{\alpha} B^{\dagger} Y^{-1} \partial_{\beta} B\right] .
$$

For the $U(N)$ theory this reduces to the $\theta$-term in the worldsheet $\mathbb{C} P^{N-1}$ sigma model, discussed by Gorsky et. al. [8]. Thus the total effective Lagrangian becomes

$$
\begin{aligned}
\mathcal{L} & =\operatorname{Tr}\left[\left(\frac{4 \pi}{g^{2}} \eta^{\alpha \beta}-i \frac{\theta}{2 \pi} \epsilon^{\alpha \beta}\right) X^{-1} \partial_{\alpha} B^{\dagger} Y^{-1} \partial_{\beta} B-\frac{4 \pi}{g^{2}} X^{-1}\left\{M_{n}, B^{\dagger}\right\} Y^{-1}\left\{M_{n}, B\right\}\right] \\
& =\operatorname{Tr}\left[\operatorname{Im}\left(\tau X^{-1} \partial_{-} B^{\dagger} Y^{-1} \partial_{+} B\right)-\frac{4 \pi}{g^{2}} X^{-1}\left\{M_{n}, B^{\dagger}\right\} Y^{-1}\left\{M_{n}, B\right\}\right],
\end{aligned}
$$

where $\tau$ is the complex coupling constant

$$
\tau \equiv \frac{\theta}{2 \pi}+i \frac{4 \pi}{g^{2}}, \quad \partial_{ \pm} \equiv \partial_{t} \pm \partial_{x_{3}} .
$$

Although the $\theta$-term (5.2) does not change the equation of motion, it shifts the conserved Noether charges of the $U(1)^{n}$ global symmetry $\left(\mathbf{H}_{n}\right.$ is defined in Eq. (4.9) $)$

$$
\begin{aligned}
\mathbf{q}= & i \frac{4 \pi}{g^{2}} \int d x_{3} \operatorname{Tr}\left[X^{-1} \partial_{t} B^{\dagger} Y^{-1}\left\{\mathbf{H}_{n}, B\right\}-X^{-1}\left\{\mathbf{H}_{n}, B^{\dagger}\right\} Y^{-1} \partial_{t} B\right] \\
& -\frac{\theta}{2 \pi} \int d x_{3} \operatorname{Tr}\left[X^{-1} \partial_{x_{3}} B^{\dagger} Y^{-1}\left\{\mathbf{H}_{n}, B\right\}+X^{-1}\left\{\mathbf{H}_{n}, B^{\dagger}\right\} Y^{-1} \partial_{x_{3}} B\right] .
\end{aligned}
$$

Recalling Eqs. (4.11) and (4.21), the shift of the $U(1)^{n}$ charges, can be written as

$$
\Delta \mathbf{q}=-\frac{\theta}{2 \pi} \mathbf{g}
$$

By using Eq. (4.16) the shift of the $U(1)_{M}$ charge can be expressed as

$$
\Delta(\mathbf{m} \cdot \mathbf{q})=-\frac{\theta}{2 \pi} \int d x_{3} \partial_{x_{3}} \sigma
$$


These represent the Witten effect [46] for the monopoles on the vortex worldsheet.

Now let us consider the dyonic configuration. For given values of the conserved Noether charge $\mathbf{q}$ and the topological charge $\mathbf{g}$, the energy can be rewritten as

$$
\begin{aligned}
E= & \frac{4 \pi}{g^{2}} \int d x_{3} \operatorname{Tr}\left[X^{-1}\left(\partial_{t} B^{\dagger}+i \sin \mu\left\{M_{n}, B^{\dagger}\right\}\right) Y^{-1}\left(\partial_{t} B-i \sin \mu\left\{M_{n}, B\right\}\right)\right] \\
+ & \frac{4 \pi}{g^{2}} \int d x_{3} \operatorname{Tr}\left[X^{-1}\left(\partial_{x_{3}} B^{\dagger}-\cos \mu\left\{M_{n}, B^{\dagger}\right\}\right) Y^{-1}\left(\partial_{x_{3}} B-\cos \mu\left\{M_{n}, B\right\}\right)\right] \\
+ & \frac{4 \pi}{g^{2}} \mathbf{m} \cdot \mathbf{g} \cos \mu+\mathbf{m} \cdot\left(\mathbf{q}+\frac{\theta}{2 \pi} \mathbf{g}\right) \sin \mu .
\end{aligned}
$$

The quantity in the third line is extremized by

$$
\tan \mu=\frac{g^{2}}{4 \pi}\left(\frac{\mathbf{m} \cdot \mathbf{q}}{\mathbf{m} \cdot \mathbf{g}}+\frac{\theta}{2 \pi}\right)
$$

Therefore, the energy is bounded from below by the central charge as

$$
E \geq \sqrt{\left[\frac{4 \pi}{g^{2}} \mathbf{m} \cdot \mathbf{g}\right]^{2}+\left[\mathbf{m} \cdot\left(\mathbf{q}+\frac{\theta}{2 \pi} \mathbf{g}\right)\right]^{2}}=|\mathbf{m} \cdot(\mathbf{q}+\tau \mathbf{g})|=|Z| .
$$

The inequality (5.10) is saturated if the following BPS equations are satisfied

$$
\partial_{t} B-i \sin \mu\left\{M_{n}, B\right\}=0, \quad \partial_{x_{3}} B-\cos \mu\left\{M_{n}, B\right\}=0
$$

The general solution is given by

$$
B\left(t, x_{3}\right)=\exp \left[M_{n}\left(i t \sin \mu+x_{3} \cos \mu\right)\right] B_{0} \exp \left[M_{n}\left(i t \sin \mu+x_{3} \cos \mu\right)\right]
$$

We can also express this solution in terms of the matrix $\Lambda$

$$
\Lambda\left(t, x_{3}\right)=\left(B_{0} \mid \mathbf{1}_{n}\right) \exp \left[M_{n}\left(i t \sin \mu+x_{3} \cos \mu\right)\right]=\Lambda_{0} \exp \left[M_{n}\left(i t \sin \mu+x_{3} \cos \mu\right)\right] .
$$

As an example, let us take the matrix $\Lambda_{0}$ for the single monopole configuration

$$
\begin{aligned}
\Lambda\left(t, x_{3}\right) & =\Lambda_{h} \exp \left[b E_{-\boldsymbol{\alpha}}\right] \exp \left[M\left(i t \sin \mu+x_{3} \cos \mu\right)\right] \\
& \sim \Lambda_{h} \exp \left[b \exp \left[\mathbf{m} \cdot \boldsymbol{\alpha}\left(i t \sin \mu+x_{3} \cos \mu\right)\right] E_{-\boldsymbol{\alpha}}\right]
\end{aligned}
$$

Since $\arg b$ is the phase moduli of the monopole, this dyonic solution can be interpreted as the $\tilde{\boldsymbol{\alpha}}$-monopole with a rotating phase

$$
\eta(t)=\arg b+\sin \mu(\mathbf{m} \cdot \boldsymbol{\alpha}) t
$$

We have seen in the previous section that the monopole phase transforms under the $U(1)^{n}$ symmetry as $\eta \rightarrow \eta+\boldsymbol{\theta} \cdot \boldsymbol{\alpha}$. This transformation property implies that the conjugate momentum of 
the phase $p_{\eta}$ and the Noether charge $\mathbf{q}$ are related by $\mathbf{q}=p_{\eta} \boldsymbol{\alpha}$. Upon semi-classical quantization of the monopole moduli, the conjugate momentum of the phase, which has period $2 \pi$, is quantized as $p_{\eta} \in \mathbb{Z}$. Therefore, the Noether charge $\mathbf{q}$ should be an integer multiple of the root $\boldsymbol{\alpha}$

$$
\mathbf{q}=n_{q} \boldsymbol{\alpha}, \quad n_{q} \in \mathbb{Z} .
$$

The BPS spectrum of the monopoles and dyons with the magnetic charge $\mathbf{g}=2 \tilde{\boldsymbol{\alpha}}$ is therefore given by

$$
M_{\boldsymbol{\alpha}, n_{q}}=\left|\mathbf{m} \cdot \boldsymbol{\alpha}\left(n_{q}+\frac{4}{\boldsymbol{\alpha} \cdot \boldsymbol{\alpha}} \tau\right)\right|
$$

This spectrum is invariant under a $2 \pi$ rotation of the $\theta$-angle $(\tau \rightarrow \tau+1)$ since the length of the root vectors are normalized by

$$
\frac{4}{\boldsymbol{\alpha} \cdot \boldsymbol{\alpha}}= \begin{cases}1 & \text { for the long roots of } U S p(2 n) \\ 2 & \text { for the other roots }\end{cases}
$$

Note that the Noether charge q cannot be interpreted as the electric charge of a dyon, whether or not it gets Witten's correction due to the vacuum angle $\theta$. This is because the electric flux is screened by the scalar field $Q$ and decays with the Yukawa type behavior $e^{-g v x}$, so that the total electric charge should be zero. On the other hand, the scalar field is charged under the unbroken global $U(1)^{n}$ symmetry and accordingly the vector $\mathbf{q}$ can be interpreted as the associated conserved charges. Indeed, we can see that the global $U(1)^{n}$ charges contribute to the BPS mass by rewriting the energy in the following way:

$$
\begin{aligned}
E=\int d^{3} x \operatorname{Tr}\left[\frac{1}{g^{2}}\left|B_{i}-\cos \mu \mathcal{D}_{i} \Phi-\delta_{i 3} g^{2}\left(\operatorname{Tr}\left[Q^{\dagger} t^{\alpha} Q\right] t^{\alpha}-\xi t^{0}\right)\right|^{2}+\frac{1}{g^{2}}\left|E_{i}-\sin \mu \mathcal{D}_{i} \Phi\right|^{2}\right. \\
+4\left|\mathcal{D}_{\bar{z}} Q\right|^{2}+\left|\mathcal{D}_{3} Q+\cos \mu(\Phi Q-Q M)\right|^{2}+\left|\mathcal{D}_{0} Q+i \sin \mu(\Phi Q-Q M)\right|^{2} \\
+\frac{1}{g^{2}}\left|\mathcal{D}_{0} \Phi\right|^{2}-\sin \mu \Phi\left(\frac{2}{g^{2}} \mathcal{D}_{i} E_{i}+\frac{i 2}{g^{2}}\left[\Phi, \mathcal{D}_{0} \Phi\right]+i\left[Q\left(\mathcal{D}_{0} Q\right)^{\dagger}-\mathcal{D}_{0} Q Q^{\dagger}\right]\right) \\
\left.-v^{2} B_{3}+\frac{2}{g^{2}} \mathcal{D}_{i}\left[\Phi B_{i}\right] \cos \mu+\sin \mu\left(\frac{2}{g^{2}} \mathcal{D}_{i}\left[\Phi E_{i}\right]+i\left[Q M\left(\mathcal{D}_{0} Q\right)^{\dagger}-\mathcal{D}_{0} Q M Q^{\dagger}\right]\right)\right] \\
\geq \int d x_{3} T_{v}+\mathbf{m} \cdot\left[\frac{4 \pi}{g^{2}} \mathbf{g} \cos \mu+\left(\mathbf{q}_{e}+\mathbf{q}_{f}\right) \sin \mu\right]
\end{aligned}
$$

where the charges are defined by

$$
\begin{gathered}
\mathbf{g} \equiv \frac{1}{2 \pi} \int d^{3} x \partial_{i} \operatorname{Tr}\left(B_{i} \mathbf{H}\right), \quad \mathbf{q}_{e} \equiv \frac{2}{g^{2}} \int d^{3} x \partial_{i} \operatorname{Tr}\left(E_{i} \mathbf{H}\right) \\
\mathbf{q}_{f} \equiv i \int d^{3} x \operatorname{Tr}\left[Q \mathbf{H}\left(\mathcal{D}_{0} Q\right)^{\dagger}-\mathcal{D}_{0} Q \mathbf{H} Q^{\dagger}\right]
\end{gathered}
$$




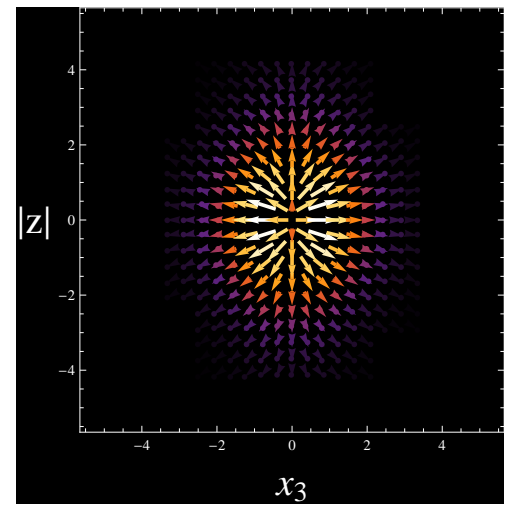

(a) $\operatorname{Tr}\left[E_{i} \Phi\right]$

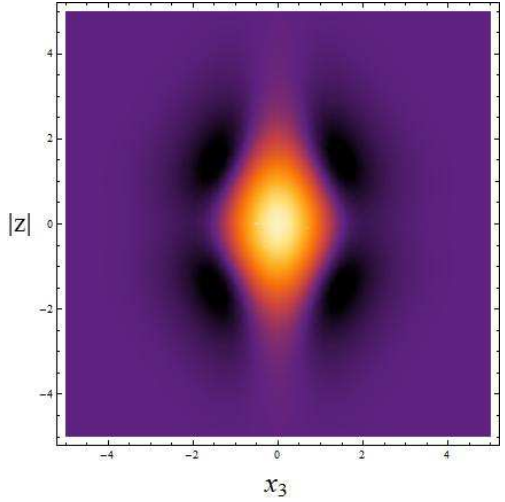

(b) $\partial_{i} \operatorname{Tr}\left[E_{i} \Phi\right]$

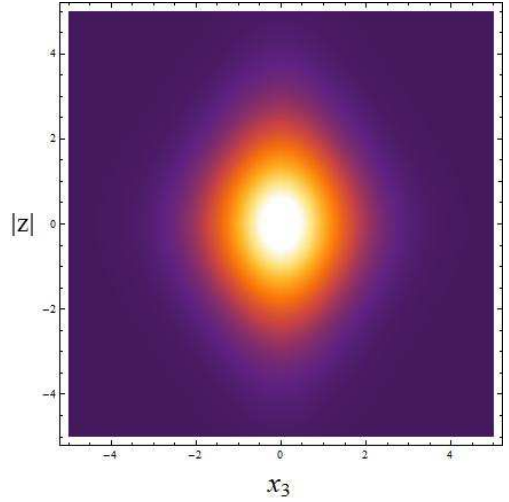

(c) $i \operatorname{Tr}\left[Q M \mathcal{D}_{0} Q^{\dagger}-\mathcal{D}_{0} Q M Q^{\dagger}\right]$

Fig. 7: (a) The electric flux of the $S U(2)$ vortex-dyon configuration. The electric flux decays with the Yukawa type behavior $e^{-g v x}$.

(b) The electric charge density.

(c) The global $U(1)$ charge. The plots for negative $|z|$ are simply mirror images in order to illustrate the cross section of the configuration.

Since the electric charge $\mathbf{q}_{e}$ should be zero for $\theta=0$ (see Fig.7), the BPS mass is given by the magnetic charge $\mathbf{g}$ and the $U(1)^{n}$ global charges $\mathbf{q}_{f}$. The relation between $\mathbf{q}$ in Eq. (5.5) and $\mathbf{q}_{f}$ in Eq. (5.20) is $\mathbf{q}=\mathbf{q}_{e}+\mathbf{q}_{f}$.

The dyonic configurations discussed in this section have parallel charge vectors: $\mathbf{q}$ and $\mathbf{g}$ are proportional to the root vectors $\boldsymbol{\alpha}$ and the coroot vectors $\tilde{\boldsymbol{\alpha}}$, respectively. We can construct dyonic configurations with $\mathbf{q} \not \propto \mathbf{g}$ if we introduce complex mass parameters

$$
M=M_{1}+i M_{2}=\left(\mathbf{m}_{1}+i \mathbf{m}_{2}\right) \cdot \mathbf{H} .
$$

By using a method similar to that discussed in section 2.3, we can easily check that the potential of the effective theory becomes

$$
V_{\mathrm{eff}}=\frac{4 \pi}{g^{2}} \sum_{a=1}^{2} \operatorname{Tr}\left[X^{-1}\left\{\mathbf{m}_{a} \cdot \mathbf{H}_{n}, B^{\dagger}\right\} Y^{-1}\left\{\mathbf{m}_{a} \cdot \mathbf{H}_{n}, B\right\}\right] .
$$

In this case, we can rewrite the energy of the effective theory as

$$
\begin{aligned}
E=\frac{4 \pi}{g^{2}} \int d x_{3} \operatorname{Tr}\left[X^{-1}\left(\partial_{x_{3}} B^{\dagger}-\left\{\tilde{\mathbf{m}}_{1} \cdot \mathbf{H}_{n}, B^{\dagger}\right\}\right) Y^{-1}\left(\partial_{x_{3}} B-\left\{\tilde{\mathbf{m}}_{1} \cdot \mathbf{H}_{n}, B\right\}\right)\right. \\
\left.+X^{-1}\left(\partial_{t} B^{\dagger}+i\left\{\tilde{\mathbf{m}}_{2} \cdot \mathbf{H}_{n}, B^{\dagger}\right\}\right) Y^{-1}\left(\partial_{t} B-i\left\{\tilde{\mathbf{m}}_{2} \cdot \mathbf{H}_{n}, B\right\}\right)\right] \\
+\frac{4 \pi}{g^{2}} \tilde{\mathbf{m}}_{1} \cdot \mathbf{g}+\tilde{\mathbf{m}}_{2} \cdot\left(\mathbf{q}+\frac{\theta}{2 \pi} \mathbf{g}\right)
\end{aligned}
$$


where $\tilde{\mathbf{m}}_{i}(i=1,2)$ are defined by

$$
\left(\begin{array}{c}
\tilde{\mathbf{m}}_{1} \\
\tilde{\mathbf{m}}_{2}
\end{array}\right)=\left(\begin{array}{cc}
\cos \mu & -\sin \mu \\
\sin \mu & \cos \mu
\end{array}\right)\left(\begin{array}{l}
\mathbf{m}_{1} \\
\mathbf{m}_{2}
\end{array}\right) .
$$

Since the quantity in the last line of Eq. (5.23) is extremized by

$$
\tan \mu=-\frac{\frac{4 \pi}{g^{2}} \mathbf{m}_{2} \cdot \mathbf{g}-\mathbf{m}_{1} \cdot\left(\mathbf{q}+\frac{\theta}{2 \pi} \mathbf{g}\right)}{\frac{4 \pi}{g^{2}} \mathbf{m}_{1} \cdot \mathbf{g}+\mathbf{m}_{2} \cdot\left(\mathbf{q}+\frac{\theta}{2 \pi} \mathbf{g}\right)},
$$

the lower bound on the energy is given by the central charge

$$
E \geq|Z|=\left|\left(\mathbf{m}_{1}+i \mathbf{m}_{2}\right) \cdot(\mathbf{g}+\tau \mathbf{q})\right|
$$

The BPS equations can be easily solved as

$$
B=e^{\left(\tilde{\mathbf{m}}_{1} x_{3}+i \tilde{\mathbf{m}}_{2} t\right) \cdot \mathbf{H}_{n}} B_{0} e^{\left(\tilde{\mathbf{m}}_{1} x_{3}+i \tilde{\mathbf{m}}_{2} t\right) \cdot \mathbf{H}_{n}} .
$$

The $n$-by- $n$ constant matrix $B_{0}$ is related to the conserved charge $\mathbf{q}$ via Eq. (5.5)). The right hand side of Eq. (5.5) is a function of the kink positions and phases contained in $B_{0}$, so that the parameters should obey some constraints determined by the conserved charge $\mathbf{q}$. This means that some of the kinks form bound states, which correspond to the 1/4 BPS dyons [47] attached to vortex strings. We can also show that if the angle between $\mathbf{g}$ and $\mathbf{q}$ takes on a generic value, the right hand side of Eq. (5.5) has an upper bound determined by the mass parameters. Therefore, there is a finite number of elements of the root lattice $\mathbf{q}$ in a generic direction 19 and the number of BPS bound states changes as we vary the mass parameters. This is also analogous to the case of the corresponding $4 d$ theory. The composite configurations should be $1 / 4$ BPS [48] while explicit checks remain a future problem.

\section{Summary}

In this paper we have studied the effects of mass deformation on the vortex effective worldsheet actions in $U(1) \times G$ gauge theories with $G=S O(2 n), U S p(2 n)$, and $S U(N)$. The moduli spaces of the vortex solutions, $S O(2 n) / U(n), U S p(2 n) / U(n)$, and $S U(N) / U(N-1) \sim \mathbb{C} P^{N-1}$, respectively, arising from the exact color-flavor symmetries broken by vortex configurations, are replaced under mass deformation by a finite number of minimum-tension vortex solutions (vortex vacua). Kinks develop along the vortex string, connecting different vortex vacua, which turn out

\footnotetext{
${ }^{19}$ Note that there exist infinite towers of the conserved charge vector $\mathbf{q}$ in some specific directions such as $\mathbf{q} \propto \mathrm{g}$.
} 
to be the ordinary three-dimensional monopoles trapped inside the vortex core. The structure of these kinks (monopoles) have been analyzed systematically here (see also [29]).

In the case of the $U(N)$ theory this $2 d-4 d$ correspondence was shown to survive quantum mechanically in the theory with no mass deformation [5, 8], thus providing a highly non-trivial realization of the $2 d-4 d$ duality proposed earlier by Dorey et. al. [21]. In the case explicitly examined from this point of view, the massless $U(1) \times S U(N)$ theory with $N_{\mathrm{f}}=N$ flavors, the vortex $\mathbb{C} P^{N-1}$ fluctuations dynamically Abelianize at low energies, in perfect agreement [24] with the physics of quantum $r=0$ vacuum of four-dimensional $\mathcal{N}=2$ SQCD [22]. In the case of $S O(2 n)$ and $U S p(2 n)$ gauge theories such a check is the subject of a future study.

We have studied in this paper the properties of local (i.e., ANO-like) vortices in $S O(2 n) \times U(1)$ and $U S p(2 n) \times U(1)$ gauge theories with $N_{\mathrm{f}}=2 n$ flavors in the color-flavor locked vacuum, Eq.(2.5). It has been noted [15, 16] that in theories other than $S U(N)$, the system possesses more general types of vortex solutions, such as fractional or semi-local vortices (vortex moduli), even with the minimum number of flavors needed for the system to possess the color-flavor locked phase, Eq. (2.5) . Furthermore, the system possesses a large class of vacua besides the particular vacuum considered here, Eq. (2.5): the above mentioned general class of vortex solutions is related to the existence of such nontrivial vacuum moduli. This means that domain walls can also be formed connecting different vacua. Accordingly, equations (2.6)-(2.9) themselves admit more general classes of solutions involving, e.g., both domain walls and vortices connecting them, such as those studied in Refs. [19, 20, 30]. The mass deformation of semi-local vortices induces domain walls in the bulk in general, though this is not always the case. A related comment is given in Appendix A.

\section{Acknowledgments}

The work of M. E. is supported in part by Grant-in Aid for Scientific Research (No. 23740226). S. B. G gratefully acknowledges a Golda Meir post-doctoral fellowship. The work of Y. J. is supported by the NSF of China under Grant No.10875129. The work of M. N. is supported in part by Grant-in Aid for Scientific Research (No. 23740198) and by the "Topological Quantum Phenomena" Grant-in Aid for Scientific Research on Innovative Areas (No. 23103515) from the Ministry of Education, Culture, Sports, Science and Technology (MEXT) of Japan. K. K. thanks IPMU and Keio University for hospitality. 


\section{A Domain wall}

As described in the main text, the 1/4 BPS equations (2.6)-(2.9) admit domain walls and vortices stretched between domain walls [19, 20, 30], when the VEVs of the scalar fields are different at $x_{3} \rightarrow-\infty$ and $x_{3} \rightarrow \infty$. In that case, the third term in Eq. (2.10) gives the domain wall charge. In the case of $G=S O(2 n), U S p(2 n)$ with $N_{f}=2 n$ and non-degenerate mass matrix, $M$, there exist many isolated $r$-vacua where a subgroup of $G$ remains unbroken, in addition to the Higgs vacua in Eq. (2.5). Therefore we need a special condition for prohibiting the creation of domain walls interpolating their vacua as a $1 / 4$ BPS solution. To illustrate this, it is convenient to consider the following $G$-invariant quantity which parametrizes vacuum moduli for the massless case $M=0$,

$$
R=\frac{2 n}{\operatorname{tr}\left(J^{\dagger} Q^{\mathrm{T}} J Q\right)} Q^{\mathrm{T}} J Q
$$

The first two equations in the $1 / 4$ BPS equations can be easily solved in terms of the invariant $R$ as

$$
0=\partial_{\bar{z}} R, \quad 0=\partial_{3} R-M^{\mathrm{T}} R-R M, \quad \Rightarrow \quad R=e^{M^{\mathrm{T}} x_{3}} R_{0}(z) e^{M x_{3}},
$$

where we have used the fact that $M$ is an element of $s o(2 n), u s p(2 n)$, that is $M^{\mathrm{T}} J+J M=0$. In the case of $M=0, R=R_{0}(z)$ is the so-called rational map describing lump (semi-local vortex) solutions. Here, we require that a composite state becomes the vacuum given by Eq. (2.5) at $\left|x_{3}\right|=|z| \rightarrow \infty$, that is, $R \rightarrow J$. According to the above solution, this requirement can be formulated more strictly as

$$
M^{\mathrm{T}} R+R M=0
$$

Local vortices, which defined by $R=J$, obviously satisfies this condition, and vortices discussed in this paper are only of local type. A generic solution for the condition is a set of fractional vortices with vanishing size moduli, $R_{0}(z)_{a b}=r_{a}(z) J_{a b}=r_{b}(z) J_{a b}$. A single local vortex is formed by coincident fractional vortices, $r_{a}(z)=r_{b}(z), a \neq b$. One way to guarantee the solution to satisfy the above condition is to require invariance of the solution under the rotation $z \rightarrow e^{i \theta} z$. This forces $R_{0}(z)$ to be constant: this turns out to be the condition for vortices to be local (see Eq. (35) of [14]). According to the principle of symmetric criticality [54], this property guarantees consistency of the low-energy effective action on the local vortices discussed in this paper. If such a condition is not met, on the other hand, it is inevitable for non-vanishing size moduli of vortices to increase indefinitely along the vortex string and create a domain wall bending logarithmically. 


\section{B Lie algebra}

In this appendix, we summarize the conventions for the Lie algebra used in this paper. The root vectors of $G=S U(N), S O(2 n), U S p(2 n)$ and $S O(2 n+1)$ are given in Table 2. The generators

\begin{tabular}{|c|ll|ll|}
\hline$G$ & positive roots & simple roots & \\
\hline$S U(N)$ & $\boldsymbol{e}_{i}-\boldsymbol{e}_{j}$ & $(1 \leq i<j \leq N)$ & $\boldsymbol{e}_{i}-\boldsymbol{e}_{i+1}$ & $(i=1, \cdots, N-1)$ \\
\hline$S O(2 n)$ & $\boldsymbol{e}_{i} \pm \boldsymbol{e}_{j}$ & $(1 \leq i<j \leq n)$ & $\boldsymbol{e}_{i}-\boldsymbol{e}_{i+1}, \boldsymbol{e}_{n-1}+\boldsymbol{e}_{n}$ & $(1 \leq i<j \leq n-1)$ \\
\hline$U S p(2 n)$ & $\boldsymbol{e}_{i} \pm \boldsymbol{e}_{j}, 2 \boldsymbol{e}_{i}$ & $(1 \leq i<j \leq n)$ & $\boldsymbol{e}_{i}-\boldsymbol{e}_{i+1}, 2 \boldsymbol{e}_{n}$ & $(1 \leq i<j \leq n-1)$ \\
\hline$S O(2 n+1)$ & $\boldsymbol{e}_{i} \pm \boldsymbol{e}_{j}, \quad \boldsymbol{e}_{i}$ & $(1 \leq i<j \leq n)$ & $\boldsymbol{e}_{i}-\boldsymbol{e}_{i+1}, \boldsymbol{e}_{n}$ & $(1 \leq i<j \leq n-1)$ \\
\hline
\end{tabular}

Table 2: The root vectors of $G=S U(N), S O(2 n)$ and $U S p(2 n)$. The set of vectors $\left\{\boldsymbol{e}_{i}\right\}$ is the standard orthonormal basis $\boldsymbol{e}_{i} \cdot \boldsymbol{e}_{j}=\delta_{i j}$.

of the Lie algebra are decomposed into the standard Cartan basis: the generators of the Cartan subalgebra $\mathbf{H}=\left(H_{1}, H_{2}, \cdots, H_{r}\right),(r=\operatorname{Rank} G)$, the raising operators $E_{\boldsymbol{\alpha}}$ and the lowering operators $E_{-\boldsymbol{\alpha}}=E_{\boldsymbol{\alpha}}^{\dagger}$. Their commutation relations are given by

$$
\left[\mathbf{H}, E_{ \pm \boldsymbol{\alpha}}\right]= \pm \boldsymbol{\alpha} E_{ \pm \boldsymbol{\alpha}}, \quad\left[E_{\boldsymbol{\alpha}}, E_{-\boldsymbol{\alpha}}\right]=\tilde{\boldsymbol{\alpha}} \cdot \mathbf{H}, \quad\left[E_{ \pm \boldsymbol{\alpha}}, E_{ \pm \boldsymbol{\beta}}\right]=N_{ \pm \boldsymbol{\alpha}, \pm \boldsymbol{\beta}} E_{ \pm \boldsymbol{\alpha} \pm \boldsymbol{\beta}}
$$

where $\boldsymbol{\alpha}$ and $\boldsymbol{\beta}$ are positive root vectors and $N_{ \pm \boldsymbol{\alpha}, \pm \boldsymbol{\beta}}$ are constants. The coroot vectors $\tilde{\boldsymbol{\alpha}}$ are defined by

$$
\tilde{\boldsymbol{\alpha}} \equiv 2 \frac{\boldsymbol{\alpha}}{\boldsymbol{\alpha} \cdot \boldsymbol{\alpha}}
$$

The coroot vectors of $G$ are the root vectors of the dual group $\tilde{G}: S U(N)$ and $S O(2 n)$ are self-dual while $U S p(2 n)$ and $S O(2 n+1)$ are dual to each other. The generators of the Cartan subalgebra $\mathbf{H}$ are the diagonal matrices whose eigenvalues are the weight vectors of the corresponding representation

$$
\mathbf{H}=\left(\begin{array}{llll}
\boldsymbol{\mu}_{1} & & & \\
& \boldsymbol{\mu}_{2} & & \\
& & \ddots & \\
& & & \boldsymbol{\mu}_{N}
\end{array}\right) .
$$

\section{B.1 $G=S U(N)$}

For the fundamental representation of $S U(N)$, the weight vectors are

$$
\boldsymbol{\mu}_{i}=\boldsymbol{e}_{i}-\frac{1}{N} \sum_{i=1}^{N} \boldsymbol{e}_{i}
$$


Therefore the generators of the Cartan subalgebra $\mathbf{H}=\left(H_{1}, \cdots, H_{N}\right)$ take the form

$$
H_{1}=\left(\begin{array}{cccc}
1-\frac{1}{N} & & & \\
& -\frac{1}{N} & & \\
& & \ddots & \\
& & & -\frac{1}{N}
\end{array}\right), \quad \cdots, \quad H_{N}=\left(\begin{array}{cccc}
-\frac{1}{N} & & & \\
& \ddots & & \\
& & -\frac{1}{N} & \\
& & & 1-\frac{1}{N}
\end{array}\right) .
$$

Note that there are $N-1$ independent matrices since $\left(\boldsymbol{e}_{1}+\cdots+\boldsymbol{e}_{N}\right) \cdot \mathbf{H}=0$. For the positive root $\alpha=\boldsymbol{e}_{i}-\boldsymbol{e}_{j}(i>j)$, the raising operator $E_{\boldsymbol{\alpha}}$ and the lowering operator $E_{-\boldsymbol{\alpha}}$ are given by

$$
E_{\boldsymbol{e}_{i}-\boldsymbol{e}_{j}}=i\left(\begin{array}{cccc}
0 & & & \\
& \ddots & & \\
& & 1 & \\
& & \ddots & \\
& & & 0
\end{array}\right), \quad E_{-\left(\boldsymbol{e}_{i}-\boldsymbol{e}_{j}\right)}={ }_{j}\left(\begin{array}{cccc}
0 & & & \\
& \ddots & & \\
& 1 & \ddots & \\
& & & 0
\end{array}\right) \text {. }
$$

For the highest weight vector $\tilde{\boldsymbol{\nu}}_{h}$ of the $S U(N)$ fundamental representation, the matrix $\lambda_{h}$ is given by

$$
\lambda_{h}=\nu_{0} \mathbf{1}_{N}+\tilde{\boldsymbol{\nu}}_{h} \cdot \mathbf{H}=\left(\begin{array}{l|l}
1 & \\
\hline & \mathbf{0}_{N-1}
\end{array}\right), \quad \nu_{0}=\frac{1}{N}, \quad \tilde{\boldsymbol{\nu}}_{h}=\boldsymbol{e}_{1}-\frac{1}{N} \sum_{i=1}^{N} \boldsymbol{e}_{i} .
$$

\section{B.2 $G=S O(2 n)$}

The weight vectors of the $2 n$-dimensional representation of $S O(2 n)$ are

$$
\boldsymbol{\mu}_{i}=\boldsymbol{e}_{i}, \quad \boldsymbol{\mu}_{i+n}=-\boldsymbol{e}_{i} \quad(1<i<n) .
$$

Therefore the generators of the Cartan subalgebra $\mathbf{H}=\left(H_{1}, \cdots, H_{n}\right)$ take the form

$$
H_{i}=i_{i+n}\left(\begin{array}{c|c}
i & i+n \\
& \\
& -1
\end{array}\right)
$$

The raising operators $E_{\boldsymbol{\alpha}}$ for the positive $\operatorname{roots} \boldsymbol{\alpha}=\boldsymbol{e}_{i}-\boldsymbol{e}_{j}, \boldsymbol{e}_{i}+\boldsymbol{e}_{j},(1 \leq j<i \leq n)$ are

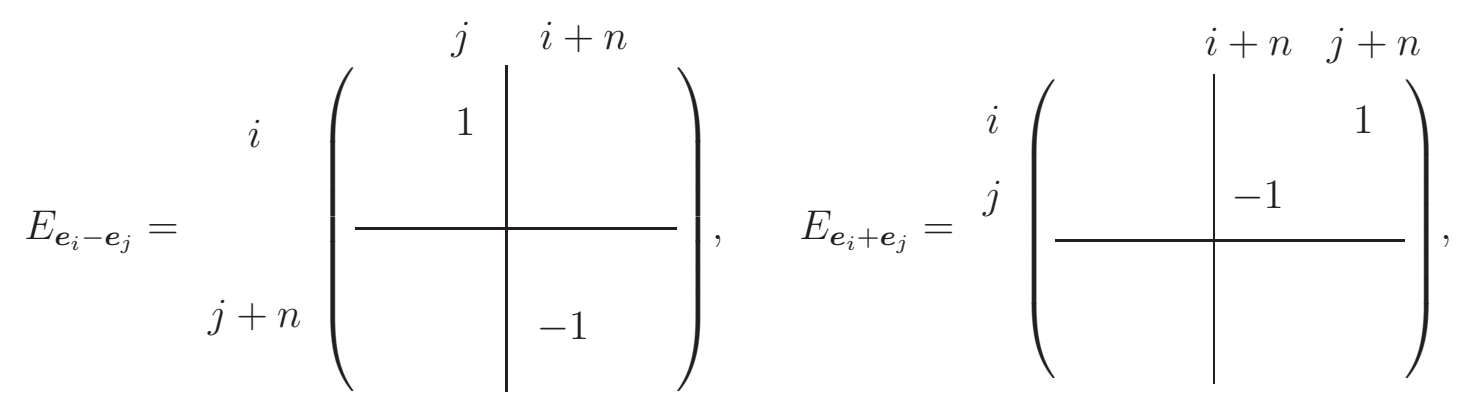


The lowering operators $E_{-\boldsymbol{\alpha}}$ for $\boldsymbol{\alpha}=\boldsymbol{e}_{i}-\boldsymbol{e}_{j}, \boldsymbol{e}_{i}+\boldsymbol{e}_{j},(1 \leq j<i \leq n)$ are

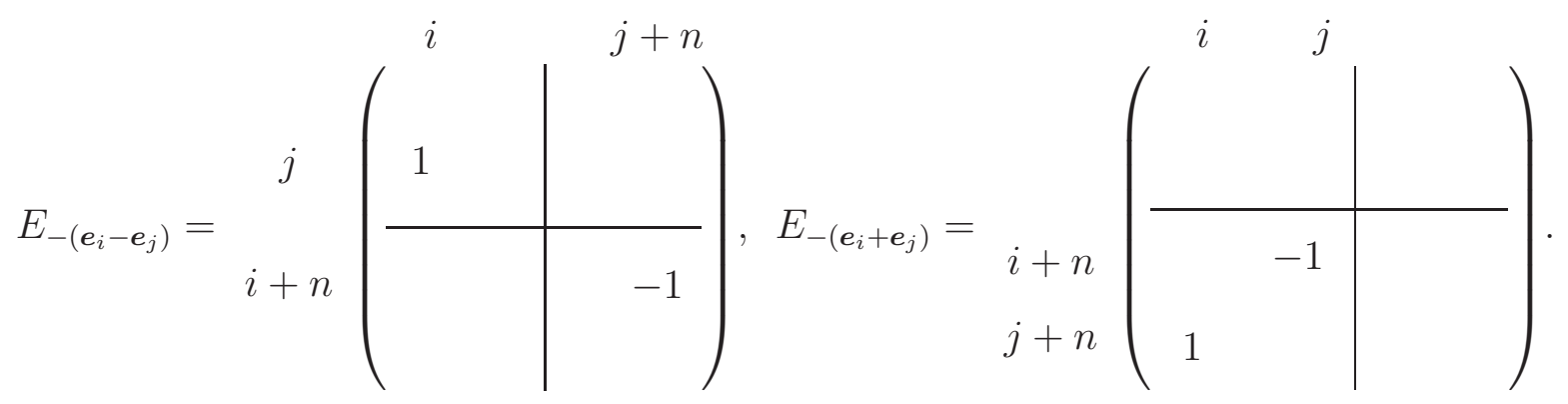

For the highest weight vector $\tilde{\boldsymbol{\nu}}_{h}$ of the $S O(2 n)$ Weyl spinor representation, the matrix $\lambda_{h}$ is given by

$$
\lambda_{h}=\nu_{0} \mathbf{1}_{2 n}+\tilde{\boldsymbol{\nu}}_{h} \cdot \mathbf{H}=\left(\begin{array}{l|l}
\mathbf{1}_{n} & \\
\hline & \mathbf{0}_{n}
\end{array}\right), \quad \nu_{0}=\frac{1}{2}, \quad \tilde{\boldsymbol{\nu}}_{h}=\frac{1}{2} \sum_{i=1}^{n} \boldsymbol{e}_{i} .
$$

\section{B.3 $G=U S p(2 n)$}

The weight vectors of the $2 n$-dimensional representation of $U S p(2 n)$ are given by

$$
\boldsymbol{\mu}_{i}=\boldsymbol{e}_{i}, \quad \boldsymbol{\mu}_{i+n}=-\boldsymbol{e}_{i} \quad(1<i<n)
$$

Therefore the generators of the Cartan subalgebra $\mathbf{H}=\left(H_{1}, \cdots, H_{n}\right)$ take the form

$$
H_{i}=i_{i+n}\left(\begin{array}{c|c}
i & i+n \\
& \\
& -1
\end{array}\right)
$$

The raising operators $E_{\boldsymbol{\alpha}}$ for the positive roots $\boldsymbol{\alpha}=\boldsymbol{e}_{i}-\boldsymbol{e}_{j}(1 \leq j<i \leq n)$ and $\boldsymbol{\alpha}=\boldsymbol{e}_{i}+\boldsymbol{e}_{j}(1 \leq$ $j \leq i \leq n)$ are

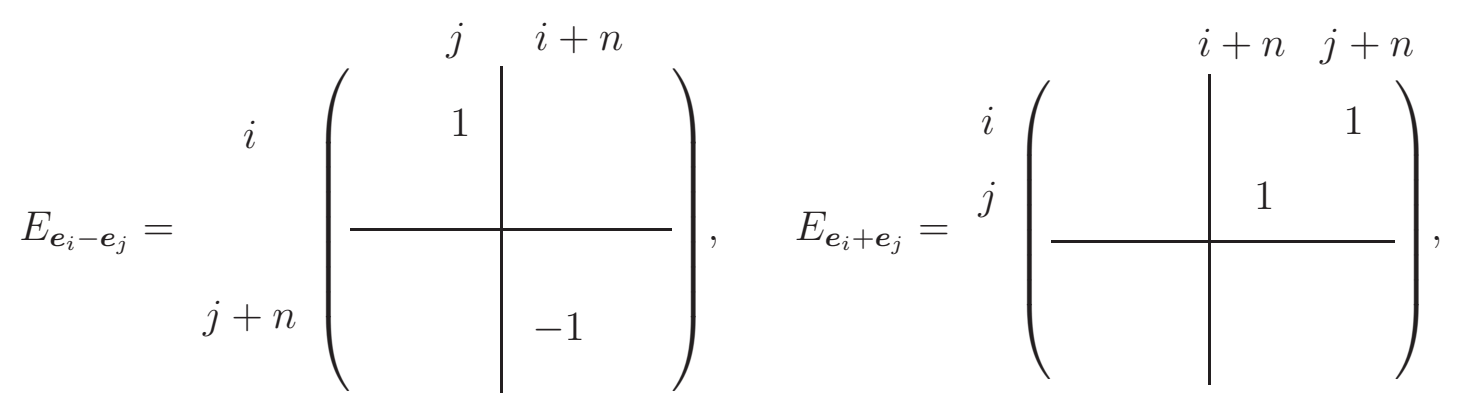


The lowering operators $E_{-\boldsymbol{\alpha}}$ for $\boldsymbol{\alpha}=\boldsymbol{e}_{i}-\boldsymbol{e}_{j}(1 \leq j<i \leq n)$ and $\boldsymbol{\alpha}=\boldsymbol{e}_{i}+\boldsymbol{e}_{j}(1 \leq j \leq i \leq n)$ are

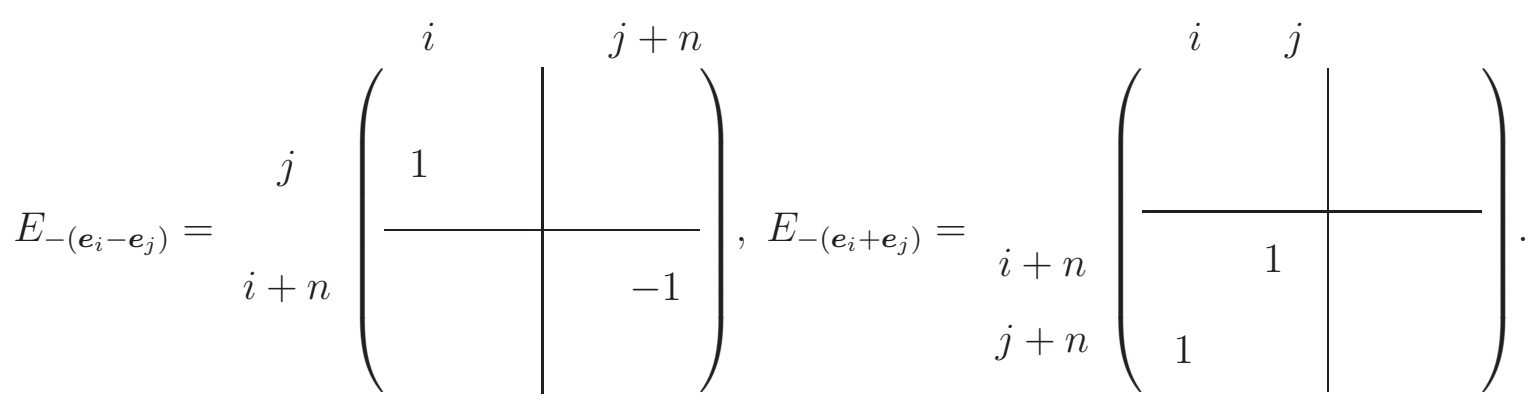

For the highest weight vector $\tilde{\boldsymbol{\nu}}_{h}$ of the $S O(2 n+1)$ spinor representation, the matrix $\lambda_{h}$ is given by

$$
\lambda_{h}=\nu_{0} \mathbf{1}_{2 n}+\tilde{\boldsymbol{\nu}}_{h} \cdot \mathbf{H}=\left(\begin{array}{l|l}
\mathbf{1}_{n} & \\
\hline & \mathbf{0}_{n}
\end{array}\right), \quad \nu_{0}=\frac{1}{2}, \quad \tilde{\boldsymbol{\nu}}_{h}=\frac{1}{2} \sum_{i=1}^{n} \boldsymbol{e}_{i} .
$$

\section{The ordering of the lowering operators}

In general, the matrix $\Lambda_{0}$ takes the following form

$$
\Lambda_{0}=\left(\Lambda_{h} w\right) \exp \left[b_{j_{1}} E_{-\boldsymbol{\alpha}_{j_{1}}}\right] \cdots \exp \left[b_{j_{q}} E_{-\boldsymbol{\alpha}_{j_{q}}}\right] R
$$

where $w$ is an element of the Weyl group and $R$ is a product of the raising operators

$$
w=w_{i_{1}} \cdots w_{i_{p}}, \quad R=\exp \left[c_{i_{1}} E_{\boldsymbol{\alpha}_{i_{1}}}\right] \cdots \exp \left[c_{i_{p}} E_{\boldsymbol{\alpha}_{i_{p}}}\right] .
$$

In this appendix, we show that the matrix $\Lambda$ can always be rewritten as

$$
\Lambda_{0} \sim\left(\Lambda_{h} w w_{j_{1}}\right) \exp \left[b_{j_{2}}^{\prime} E_{-\boldsymbol{\alpha}_{j_{2}}}\right] \cdots \exp \left[b_{j_{q}}^{\prime} E_{-\boldsymbol{\alpha}_{j_{q}}}\right] \exp \left[b_{j_{1}}^{-1} E_{\boldsymbol{\alpha}_{j_{1}}}\right] R
$$

By using the decomposition formula (4.66), we can rewrite $\Lambda_{0}$ as

$$
\Lambda_{0} \sim\left(\Lambda_{h} w w_{j_{1}}\right) \exp \left[a_{j_{1}}^{-1} E_{\boldsymbol{\alpha}_{j_{1}}}\right] \exp \left[a_{j_{2}} E_{-\boldsymbol{\alpha}_{j_{2}}}\right] \cdots \exp \left[a_{j_{q}} E_{-\boldsymbol{\alpha}_{j_{q}}}\right] R
$$

If $j_{1} \notin\left\{j_{2}, \cdots, j_{q}\right\}$, we can move the operator $\exp \left[a_{j_{1}}^{-1} E_{\boldsymbol{\alpha}_{j_{1}}}\right]$ to the right of all the lowering operators. Then we obtain the matrix $\Lambda_{0}$ of the form (C.3).

On the other hand, if there exists the same lowering operator $\left(\exists j_{r}=j_{1}\right)$, we cannot exchange the positions of the operators

$$
\exp \left[a_{j_{1}}^{-1} E_{\boldsymbol{\alpha}_{j_{1}}}\right] \exp \left[a_{j_{r}} E_{-\boldsymbol{\alpha}_{j_{1}}}\right] \neq \exp \left[a_{j_{r}} E_{-\boldsymbol{\alpha}_{j_{1}}}\right] \exp \left[a_{j_{1}}^{-1} E_{\boldsymbol{\alpha}_{j_{1}}}\right]
$$


Instead, we use the following formula

$$
\begin{aligned}
\exp \left[b_{j_{1}}^{-1} E_{\boldsymbol{\alpha}_{j_{1}}}\right] \exp \left[b_{j_{r}} E_{-\boldsymbol{\alpha}_{j_{1}}}\right] & =\exp \left[\log \left(1+b_{j_{1}}^{-1} b_{j_{r}}\right) \tilde{\boldsymbol{\alpha}}_{j_{1}} \cdot \mathbf{H}\right] \\
& \times \exp \left[b_{j_{r}}\left(1+b_{j_{1}}^{-1} b_{j_{r}}\right) E_{-\boldsymbol{\alpha}_{j_{1}}}\right] \exp \left[b_{j_{1}}^{-1}\left(1+b_{j_{1}}^{-1} b_{j_{r}}\right)^{-1} E_{\boldsymbol{\alpha}_{j_{1}}}\right] .
\end{aligned}
$$

Then the matrix $\Lambda_{0}$ can be rewritten as

$$
\begin{aligned}
\Lambda_{0} \sim\left(\Lambda_{h} w w_{j_{1}}\right) & \exp \left[b_{j_{2}}^{\prime} E_{-\boldsymbol{\alpha}_{j_{2}}}\right] \cdots \exp \left[b_{j_{r-1}}^{\prime} E_{-\boldsymbol{\alpha}_{j_{r-1}}}\right] \exp \left[a_{j_{r}}\left(1+a_{j_{1}}^{-1} a_{j_{r}}\right) E_{-\boldsymbol{\alpha}_{j_{1}}}\right] \\
& \times \exp \left[a_{j_{1}}^{-1}\left(1+a_{j_{1}}^{-1} a_{j_{r}}\right)^{-1} E_{\boldsymbol{\alpha}_{j_{1}}}\right] \exp \left[a_{j_{r+1}} E_{-\boldsymbol{\alpha}_{j_{r+1}}}\right] \cdots \exp \left[a_{j_{q}} E_{-\boldsymbol{\alpha}_{j_{q}}}\right] R
\end{aligned}
$$

where the parameters $b_{j}^{\prime}$ are given by

$$
b_{j_{i}}^{\prime}=b_{j_{i}}\left(1+b_{j_{1}}^{-1} b_{j_{r}}\right)^{\tilde{\boldsymbol{\alpha}}_{j_{1}} \cdot \boldsymbol{\alpha}_{j_{i}}} .
$$

Repeating this procedure for all $j_{i}=j_{1}$, we can move the raising operator $\exp \left[b E_{\boldsymbol{\alpha}_{j_{1}}}\right]$ to the right and consequently we obtain the matrix $\Lambda_{0}$ of the form (C.3).

\section{The quadric surface $Q^{2 n-2}$ sigma models}

As explained in detail in Ref. [9], the non-Abelian vortex in $U(1) \times S O(2 n)$ has an irreducible orbit in the higher winding $k=2$ case, which has as an effective low-energy theory on the worldsheet the sigma model on the Hermitian symmetric space $Q^{2 n-2}=\frac{S O(2 n)}{S O(2) \times S O(2 n-2)}$, which has the following Kähler potential

$$
K=2 \beta \log \left(1+2 \varphi^{\dagger} \varphi+\left|\varphi^{\mathrm{T}} \varphi\right|^{2}\right)
$$

giving rise to the Lagrangian [42, 52, 9]

$$
\mathcal{L}=8 \beta\left\{\frac{\partial_{\alpha} \varphi^{\dagger} \partial_{\alpha} \varphi+2\left|\varphi^{\mathrm{T}} \partial_{\alpha} \varphi\right|^{2}}{1+2 \varphi^{\dagger} \varphi+\left|\varphi^{\mathrm{T}} \varphi\right|^{2}}-\frac{2\left|\varphi^{\dagger} \partial_{\alpha} \varphi+\left(\varphi^{\dagger} \bar{\varphi}\right)\left(\varphi^{\mathrm{T}} \partial_{\alpha} \varphi\right)\right|^{2}}{\left[1+2 \varphi^{\dagger} \varphi+\left|\varphi^{\mathrm{T}} \varphi\right|^{2}\right]^{2}}\right\}
$$

where $\varphi$ is a complex $(2 n-2)$-component vector. The Lagrangian is symmetric under the following transformation

$$
\varphi \rightarrow U \varphi
$$

where $U^{\dagger} U=1$ and $U^{\mathrm{T}} U=1$. Choosing

$$
U=e^{i M \vartheta},
$$


it is clear that $M$ has to be Hermitian and anti-symmetric and hence purely imaginary. Now keeping only the zero mode upon compactification, we get

$$
\varphi(t, z, \vartheta)=e^{i M \vartheta} \varphi_{0}(t, z)
$$

Inserting this field into the Lagrangian (D.2) we obtain 53

$$
\mathcal{L}=8 \beta\left\{\frac{\partial_{\alpha} \varphi^{\dagger} \partial_{\alpha} \varphi+2\left|\varphi^{\mathrm{T}} \partial_{\alpha} \varphi\right|^{2}+\varphi^{\dagger} M^{2} \varphi}{1+2 \varphi^{\dagger} \varphi+\left|\varphi^{\mathrm{T}} \varphi\right|^{2}}-\frac{2\left|\varphi^{\dagger} \partial_{\alpha} \varphi+\left(\varphi^{\dagger} \bar{\varphi}\right)\left(\varphi^{\mathrm{T}} \partial_{\alpha} \varphi\right)\right|^{2}+\left|\varphi^{\dagger} M \varphi\right|^{2}}{\left[1+2 \varphi^{\dagger} \varphi+\left|\varphi^{\mathrm{T}} \varphi\right|^{2}\right]^{2}}\right\}
$$

where we have used that $\varphi^{\mathrm{T}} M \varphi=0$ due to the anti-symmetry of the mass matrix. The vacuum equations read

$$
\varphi^{\dagger} M^{2} \varphi=0, \quad \varphi^{\dagger} M \varphi=0
$$

which for a generic choice of the mass matrix yields the only solution $\varphi=0$. Hence, we find the number of vacua to be 53

$$
n_{\text {vacua }}^{S O(2 n), k=2}=2 n
$$

This result is indeed expected as this irreducible orbit of the corresponding vortex should transform as an $S O(2 n)$ vector.

\section{References}

[1] A. Hanany, D. Tong, "Vortices, instantons and branes," JHEP 0307, 037 (2003). [arXiv: hep-th/0306150.

[2] R. Auzzi, S. Bolognesi, J. Evslin, K. Konishi and A. Yung, "NonAbelian superconductors: Vortices and confinement in N=2 SQCD," Nucl. Phys. B673, 187-216 (2003). [arXiv: hep-th/0307287].

[3] D. Tong, "Monopoles in the Higgs phase," Phys. Rev. D 69 (2004) 065003 [arXiv: hep-th/0307302.

[4] R. Auzzi, S. Bolognesi, J. Evslin and K. Konishi, "Nonabelian monopoles and the vortices that confine them," Nucl. Phys. B 686, 119 (2004) [arXiv: hep-th/0312233].

[5] A. Hanany and D. Tong, "Vortex strings and four-dimensional gauge dynamics," JHEP 0404, 066 (2004) [arXiv: hep-th/0403158]. 
[6] M. Shifman, A. Yung, "NonAbelian string junctions as confined monopoles," Phys. Rev. D70, 045004 (2004). [arXiv: hep-th/0403149].

[7] M. Eto, Y. Isozumi, M. Nitta, K. Ohashi, N. Sakai, "Instantons in the Higgs phase," Phys. Rev. D72, 025011 (2005). [arXiv: hep-th/0412048].

[8] A. Gorsky, M. Shifman, A. Yung, "Non-Abelian meissner effect in Yang-Mills theories at weak coupling," Phys. Rev. D71, 045010 (2005). [arXiv: hep-th/0412082].

[9] S.B. Gudnason, Y. Jiang and K. Konishi, "Non-Abelian vortex dynamics: Effective worldsheet action", JHEP 1008:012 (2010) [arXiv: 1007.2116 [hep-th]].

[10] M. Eto, Y. Isozumi, M. Nitta, K. Ohashi and N. Sakai, "Moduli space of non-Abelian vortices," Phys. Rev. Lett. 96, 161601 (2006) [arXiv: hep-th/0511088]; M. Eto, K. Konishi, G. Marmorini, M. Nitta, K. Ohashi, W. Vinci, N. Yokoi, "Non-Abelian Vortices of Higher Winding Numbers," Phys. Rev. D74, 065021 (2006). hep-th/0607070.

[11] M. Eto, K. Hashimoto, G. Marmorini, M. Nitta, K. Ohashi and W. Vinci, "Universal Reconnection of Non-Abelian Cosmic Strings," Phys. Rev. Lett. 98, 091602 (2007) arXiv:hep-th/0609214.

[12] T. Fujimori, G. Marmorini, M. Nitta, K. Ohashi and N. Sakai, "The Moduli Space Metric for Well-Separated Non-Abelian Vortices," Phys. Rev. D 82, 065005 (2010) arXiv:1002.4580 [hep-th]].

[13] M. Eto, T. Fujimori, M. Nitta, K. Ohashi and N. Sakai, "Dynamics of Non-Abelian Vortices," arXiv:1105.1547 [hep-th].

[14] M. Eto, T. Fujimori, S. B. Gudnason, K. Konishi, M. Nitta, K. Ohashi and W. Vinci, "Constructing Non-Abelian Vortices with Arbitrary Gauge Groups," Phys. Lett. B 669, 98 (2008) [arXiv: 0802.1020 [hep-th]].

[15] M. Eto, T. Fujimori, S. B. Gudnason, M. Nitta, K. Ohashi, "SO and USp Kähler and Hyper-Kähler Quotients and Lumps," Nucl. Phys. B815, 495-538 (2009) arXiv:0809.2014 [hep-th]].

[16] M. Eto, T. Fujimori, S. B. Gudnason, K. Konishi, T. Nagashima, M. Nitta, K. Ohashi and W. Vinci, "Non-Abelian Vortices in $\mathrm{SO}(\mathrm{N})$ and $\mathrm{USp}(\mathrm{N})$ Gauge Theories," JHEP 0906, 004 (2009) [arXiv: 0903.4471 [hep-th]]. 
[17] Y. Isozumi, M. Nitta, K. Ohashi, N. Sakai, "Construction of non-Abelian walls and their complete moduli space," Phys. Rev. Lett. 93, 161601 (2004) [arXiv: hep-th/0404198].

[18] Y. Isozumi, M. Nitta, K. Ohashi, N. Sakai, "Non-Abelian walls in supersymmetric gauge theories," Phys. Rev. D70, 125014 (2004) hep-th/0405194].

[19] Y. Isozumi, M. Nitta, K. Ohashi, N. Sakai, "All exact solutions of a 1/4 Bogomol'nyi-PrasadSommerfield equation," Phys. Rev. D71, 065018 (2005). [arXiv: hep-th/0405129].

[20] M. Eto, Y. Isozumi, M. Nitta, K. Ohashi and N. Sakai, "Solitons in the Higgs phase: The moduli matrix approach," J. Phys. A 39 (2006) R315 arXiv:hep-th/0602170.

[21] N. Dorey, "The BPS spectra of two-dimensional supersymmetric gauge theories with twisted mass terms," JHEP 9811 (1998) 005 [arXiv: hep-th/9806056]; N. Dorey, T. J. Hollowood and D. Tong, "The BPS spectra of gauge theories in two and four dimensions," JHEP 9905 (1999) 006 [arXiv: hep-th/9902134].

[22] G. Carlino, K. Konishi, H. Murayama, "Dynamics of supersymmetric SU(n(c)) and USp $(2 n(c))$ gauge theories," JHEP 0002 (2000) 004 [arXiv: hep-th/0001036]; "Dynamical symmetry breaking in supersymmetric $\operatorname{SU}(\mathrm{n}(\mathrm{c}))$ and $\operatorname{USp}(2 \mathrm{n}(\mathrm{c}))$ gauge theories," Nucl. Phys. B 590, 37 (2000). [arXiv: hep-th/0005076].

[23] S. Bolognesi, K. Konishi, G. Marmorini, "Light non-Abelian monopoles and generalized r-vacua in supersymmetric gauge theories," Nucl. Phys. B 718, 134 (2005) [arXiv: hep-th/0502004.

[24] D. Dorigoni, K. Konishi and K. Ohashi, "Non-Abelian vortices with product moduli," Phys.Rev. D79 (2009) 045011 [arXiv: 0801.3284 [hep-th]].

[25] M. Eto, T. Fujimori, S. B. Gudnason, Y. Jiang, K. Konishi, M. Nitta and K. Ohashi, "Group Theory of Non-Abelian Vortices", JHEP 1011 (2010) 042 [arXiv:1009.4794 [hep-th]].

[26] J. Scherk and J. H. Schwarz, "Spontaneous Breaking Of Supersymmetry Through Dimensional Reduction," Phys. Lett. B 82 (1979) 60.

[27] J. Scherk and J. H. Schwarz, "How To Get Masses From Extra Dimensions," Nucl. Phys. B 153 (1979) 61.

[28] N. Sakai and D. Tong, "Monopoles, vortices, domain walls and D-branes: The Rules of interaction," JHEP 0503, 019 (2005) arXiv:hep-th/0501207]. 
[29] M. Arai and S. Shin, "Walls of massive Kähler sigma models on $\mathrm{SO}(2 \mathrm{~N}) / \mathrm{U}(\mathrm{N})$ and Sp(N)/U(N)," Phys. Rev. D 83, 125003 (2011) [arXiv:1103.1490 [hep-th]].

[30] M. Eto, T. Fujimori, T. Nagashima, M. Nitta, K. Ohashi and N. Sakai, "Dynamics of Strings between Walls," Phys. Rev. D 79, 045015 (2009) [arXiv:0810.3495 [hep-th]].

[31] M. Eto, T. Fujimori, S. B. Gudnason, K. Konishi, T. Nagashima, M. Nitta, K. Ohashi, W. Vinci, Phys. Rev. D80, 045018 (2009). [arXiv:0905.3540 [hep-th]].

[32] F. Delduc and G. Valent, "Classical And Quantum Structure Of The Compact Kahlerian Sigma Models," Nucl. Phys. B 253, 494 (1985); F. Delduc and G. Valent, "Renormalizability Of The Generalized Sigma Models Defined On Compact Hermitian Symmetric Spaces," Phys. Lett. B 148 (1984) 124.

[33] M. Eto, Y. Isozumi, M. Nitta, K. Ohashi, N. Sakai, "Manifestly supersymmetric effective Lagrangians on BPS solitons," Phys. Rev. D73, 125008 (2006). hep-th/0602289].

[34] M. Shifman and A. Yung, "Localization of nonAbelian gauge fields on domain walls at weak coupling (D-brane prototypes II)," Phys. Rev. D 70, 025013 (2004) arXiv:hep-th/0312257]; M. Eto, M. Nitta, K. Ohashi and D. Tong, "Skyrmions from instantons inside domain walls," Phys. Rev. Lett. 95, 252003 (2005) arXiv:hep-th/0508130]; M. Eto, T. Fujimori, M. Nitta, K. Ohashi, N. Sakai, "Domain walls with non-Abelian clouds," Phys. Rev. D77, 125008 (2008). arXiv:0802.3135 [hep-th]].

[35] M. Nitta, W. Vinci, "Non-Abelian Monopoles in the Higgs Phase," Nucl. Phys. B848, 121-154 (2011). arXiv:1012.4057 [hep-th]].

[36] L. Alvarez-Gaume and D. Z. Freedman, "Potentials for the Supersymmetric Nonlinear Sigma Model," Commun. Math. Phys. 91, 87 (1983).

[37] S. J. J. Gates, "Superspace Formulation of New Nonlinear Sigma Models," Nucl. Phys. B 238, 349 (1984).

[38] D. Tong, "A Note on 1/4 BPS states," Phys. Lett. B460, 295-301 (1999) hep-th/9902005.

[39] E. R. C. Abraham and P. K. Townsend, "Q kinks," Phys. Lett. B 291, 85 (1992); "More on Q kinks: A (1+1)-dimensional analog of dyons," Phys. Lett. B 295, 225 (1992); M. Arai, M. Naganuma, M. Nitta, N. Sakai, "Manifest supersymmetry for BPS walls in N=2 nonlinear sigma models," Nucl. Phys. B652, 35-71 (2003) hep-th/0211103. 
[40] J. P. Gauntlett, D. Tong and P. K. Townsend, "Multidomain walls in massive supersymmetric sigma models," Phys. Rev. D 64, 025010 (2001) arXiv:hep-th/0012178; D. Tong, "The Moduli space of BPS domain walls," Phys. Rev. D 66, 025013 (2002) arXiv:hep-th/0202012.

[41] E. Witten, "Dynamical Breaking of Supersymmetry," Nucl. Phys. B188, 513 (1981); E. Witten, "Constraints on Supersymmetry Breaking," Nucl. Phys. B202, 253 (1982).

[42] K. Higashijima and M. Nitta, "Supersymmetric nonlinear sigma models as gauge theories," Prog. Theor. Phys. 103, 635 (2000) arXiv:hep-th/9911139.

[43] M. Eto, Y. Isozumi, M. Nitta, K. Ohashi, K. Ohta and N. Sakai, "D-brane construction for non-Abelian walls," Phys. Rev. D 71, 125006 (2005) arXiv:hep-th/0412024.

[44] M. Arai, M. Nitta and N. Sakai, "Vacua of massive hyperKahler sigma models of nonAbelian quotient," Prog. Theor. Phys. 113, 657 (2005) arXiv:hep-th/0307274.

[45] M. Eto, T. Fujimori, M. Nitta, K. Ohashi, K. Ohta and N. Sakai, "Statistical mechanics of vortices from D-branes and T-duality," Nucl. Phys. B 788, 120 (2008) arXiv:hep-th/0703197.

[46] E. Witten, "Dyons of Charge e theta/2 pi," Phys. Lett. B86, 283-287 (1979).

[47] K. Hashimoto, H. Hata and N. Sasakura, "Three - string junction and BPS saturated solutions in SU(3) supersymmetric Yang-Mills theory," Phys. Lett. B 431, 303 (1998) arXiv:hep-th/9803127; "Multipronged strings and BPS saturated solutions in SU(N) supersymmetric Yang-Mills theory," Nucl. Phys. B 535, 83 (1998) arXiv:hep-th/9804164; K. -M. Lee, P. Yi, "Dyons in $\mathrm{N}=4$ supersymmetric theories and three pronged strings," Phys. Rev. D58, 066005 (1998). [hep-th/9804174].

[48] M. Eto, Y. Isozumi, M. Nitta and K. Ohashi, "1/2, 1/4 and 1/8 BPS equations in SUSY Yang-Mills-Higgs systems: Field theoretical brane configurations," Nucl. Phys. B 752, 140 (2006) arXiv:hep-th/0506257.

[49] M. Shifman, A. Yung, "Non-Abelian semilocal strings in N=2 supersymmetric QCD," Phys. Rev. D73, 125012 (2006) hep-th/0603134.

[50] M. Eto, J. Evslin, K. Konishi, G. Marmorini, M. Nitta, K. Ohashi, W. Vinci, N. Yokoi, "On the moduli space of semilocal strings and lumps," Phys. Rev. D76, 105002 (2007) arXiv:0704.2218 [hep-th]]. 
[51] M. Shifman, W. Vinci, A. Yung, "Effective World-Sheet Theory for Non-Abelian Semilocal Strings in N = 2 Supersymmetric QCD," Phys. Rev. D83, 125017 (2011) arXiv:1104.2077 [hep-th]].

[52] K. Higashijima, T. Kimura, M. Nitta and M. Tsuzuki, "Large N limit of N=2 supersymmetric $Q^{N}$ model in two-dimensions," Prog. Theor. Phys. 105, 261 (2001) arXiv:hep-th/0010272.

[53] M. Arai, S. Lee and S. Shin, "Walls in supersymmetric massive nonlinear sigma model on complex quadric surface," Phys. Rev. D 80, 125012 (2009) arXiv:0908.3713 [hep-th]].

[54] N. Manton and P. Sutcliffe, "Topological Solitons", Cambridge University Press, Cambridge (2004), Section 4.4; R.S. Palais, "The principle of symmetric criticality", Commun. Math. Phys., 69, 19 (1979). 